CUBO A Mathematical Journal

Vol.16, No 01, (71-110). June 2014

\title{
Trisectors like Bisectors with equilaterals instead of Points
}

\author{
SPIRIDON A. KURUKLIS \\ Eurobank, \\ Group IT Security and Risk Management Division \\ Athens, GREECE \\ skuruklis@gmail.com
}

\begin{abstract}
It is established that among all Morley triangles of $\triangle A B C$ the only equilaterals are the ones determined by the intersections of the proximal to each side of $\triangle A B C$ trisectors of either interior, or exterior, or one interior and two exterior angles. It is showed that these are in fact equilaterals, with uniform proofs. It is then observed that the intersections of the interior trisectors with the sides of the interior Morley equilateral form three equilaterals. These along with Pasch's axiom are utilized in showing that Morley's theorem does not hold if the trisectors of one exterior and two interior angles are used in its statement.
\end{abstract}

\section{RESUMEN}

Se establece que entre todos los triángulos de Morley de $\triangle A B C$, los únicos equiláteros son theones determinados por las intersecciones del proximal a cada lado de los trisectores $\triangle A B C$ de ángulos interior, o exterior, o uno interior y dos exteriores. Se muestra que estos están en triángulos equiláteros de facto con demostraciones uniformes. Luego, se observa que las intersecciones de trisectores interiores con los lados de un equilátero Morley interior forman tres triángulos equiláteros. Junto con el axioma de Pasch, se utilizan para probar que el Teorema de Morley no se satisface si se usan los trisectores de un ángulo exterior y dos interiores.

Keywords and Phrases: Angle trisection, Morley's theorem, Morley trisector theorem, Morley triangle, Morley interior equilateral, Morley central equilateral, Morley exterior equilateral, Pasch's axiom, Morley's magic, Morley's miracle, Morley's mystery.

2010 AMS Mathematics Subject Classification: 51M04 


\section{Introduction}

The systematic study of the angle trisectors in a triangle starts after 1899, when Frank Morley, a Cambridge mathematician, who had just been recently appointed professor at Haverford College, U.S.A. while investigating certain geometrical properties using abstract algebraic methods, made the following astonishing observation, known since then as Morley's theorem.

In any triangle the trisectors of its angles, proximal to the three sides respectively, meet at the vertices of an equilateral.

A Morley triangle of $\triangle \mathrm{ABC}$ is formed by the three points of intersection of pairs of angle trisectors connected by each triangle side. Obviously for a particular side there are four possibilities for pairing trisectors since there are four of them that the side connects. Thus Morley's theorem claims that a Morley triangle of $\triangle A B C$ is equilateral, if it is formed by the intersections of trisectors proximal to the three sides of $\triangle \mathrm{ABC}$ respectively.

It should be noted that Morley's theorem, as it is stated, is subject to interpretation as the term angle could mean either interior or exterior angle, or even a combination of both for the different instances of the term in the statement.

According to the angle meaning, Morley's theorem

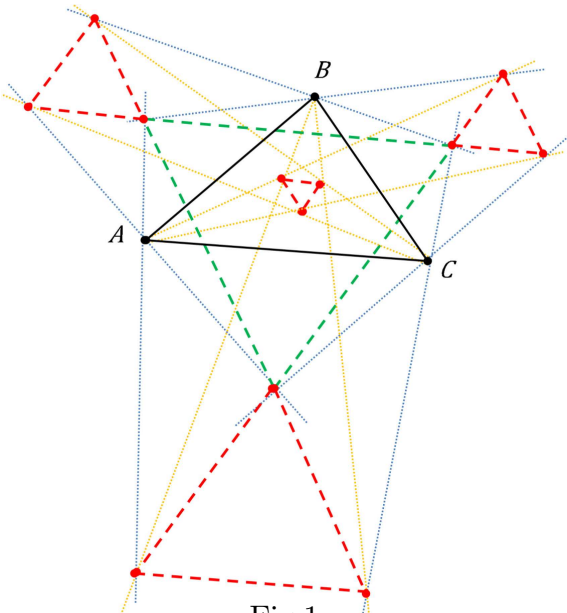

Fig.1 gives the following Morley equilaterals of $\triangle \mathrm{ABC}$. The intersections of the proximal trisectors of the interior angles form the interior Morley equilateral of $\triangle A B C$. Also the intersections of the proximal trisectors of the exterior angles form the central Morley equilateral of $\triangle A B C$. In addition the intersections of the proximal trisectors of one interior and two exterior angles form an exterior Morley equilateral of $\triangle A B C$, and thus there are three exterior Morley equilaterals of $\triangle A B C$. Fig.1 depicts the above Morley equilaterals. Proofs that the above Morley triangles are in fact equilaterals are given in Part 3 of this work.

But so an obvious question, that several authors have raised, begs for an answer. In a $\triangle \mathrm{ABC}$ are there other Morley equilaterals besides the interior, the central and the three exterior Morley equilaterals?

Apparently the requirement of Morley's theorem is satisfied by three more Morley triangles formed by combinations of proximal trisectors of an exterior and two interior angles. One of them is portrayed in Fig.2. Some experimentation using computer generated

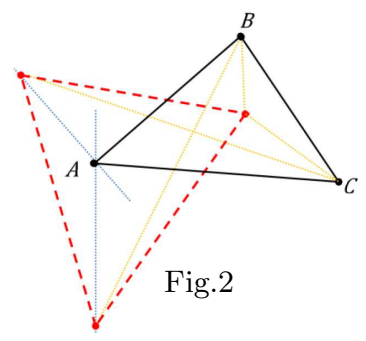
graphs for these triangles has tempted the belief that Morley's theo- 
rem holds for them as well [14]. But in Part 5, it will be proved that these are not equilaterals.

After the examination of all Morley triangles it will be shown that the equilateral ones are exactly the interior, the central and the three exterior Morley equilaterals.

This enables the establishment of an analogy between the structures of the angle bisectors and the angle trisectors in a triangle. Namely, the structure of trisectors resembles the structure of bisectors with the inner and the exterior Morley equilaterals of $\triangle A B C$ corresponding to the incenter and the excenters of $\triangle A B C$ respectively, while the central Morley equilateral corresponds to the triangle with vertices the excenters of $\triangle A B C$.

Morley's theorem is considered among the most surprising discoveries in mathematics as it went curiously unnoticed across the ages. Ancient Greeks studied the triangle geometry in depth and they could find it. But curiously they did not and it was overlooked during the following two thousand years.

Angle trisectors exist regardless of how they can be constructed. If the structure of angle trisectors maintains the regularity which characterizes the triangle geometry then theorems must exist for expressing it.

The first observation about this regularity may have forgotten. Morley didn't publish it until 25 years later by providing a sketchy proof, when the theorem had become already famous. But Morley, excited by his discovery, travelled back to England to mention it to his expert friends. In turn mathematical gossip spread it over the world and several journals proposed it for a proof.

Obviously, the simplicity of the theorem statement creates the expectation of an equally simple proof. This simplicity challenges the mathematical talent.

The vast majority of publications on Morley's theorem has treated only the trisectors of the interior angles and gave proofs for the interior Morley equilateral. In the preface of the first publication on the subject, by Taylor and Marr [12], it is recognized that the Morley's work on vector analysis, from which the above theorem follows, holds for both interior and exterior trisectors. The paper's treatment of the theorem with only the interior trisectors is explained as "Morley's work never published and it was only the particular case of internal trisectors that reached the authors". The very respectable given effort has produced proofs of many kinds, exploiting a variety of features. Trigonometric, analytic and algebraic proofs supplement the proofs of a purely geometric kind. Site Cut the Knot [13] presents 27 different proofs of Morley's theorem from many more available. Notably, Roger Penrose [9] used a tiling technique, Edsger Dijkstra applied the rule of sines three times and then the monotonicity of the function $y=\sin (x)$ in the first quadrant [3], Alain Connes offered a proof in Algebraic Geometry [1], John Conway showed it in plane geometry like a jigsaw puzzle solution [2], while Richard Guy proved that it is a consequence of his Lighthouse theorem [5]. However, a geometric, concise and logically transparent proof is still desirable. 
Richard Guy notes: "There are a few hints that there is more than one Morley triangle, but Hosberger [p. 98] asks the reader to show that Morley's theorem holds also in the case of the trisection of the exterior angles of a triangle " [5]. Rose [10] and Spickerman [11] have proposed proofs, using different methods, for the central Morley equilateral. In Parts 3 and 4 proofs for the exterior Morley equilaterals will be offered.

The most popular technique for proving Morley's theorem is encountered as indirect, backwards or reverse construction method and fits in the following scheme.

Given a triangle assume that its angles are trisected and equal to $3 \alpha, 3 \beta$ and $3 \gamma$, respectively, where $\alpha+\beta+\gamma=60^{\circ}$. In order to show that one of its Morley triangles is equilateral, start with an equilateral $\triangle \mathrm{A}^{\prime} \mathrm{B}^{\prime} \mathrm{C}^{\prime}$ and construct a $\triangle \mathrm{ABC}$ with angles $3 \alpha, 3 \beta$ and $3 \gamma$, so that $\triangle \mathrm{A}^{\prime} \mathrm{B}^{\prime} \mathrm{C}^{\prime}$ is the appropriate (interior, central or exterior) Morley triangle of $\triangle \mathrm{ABC}$. Thus $\triangle \mathrm{ABC}$ would be similar to the given triangle and so would be their corresponding Morley triangles.

Proofs of the above method most often construct $\triangle A B C$ by erecting $\triangle B^{\prime} A C^{\prime}, \triangle C^{\prime} B A^{\prime}$ and $\triangle A^{\prime} C^{\prime}$ with proper choice of the angles formed on the sides of $\triangle A^{\prime} B^{\prime} C^{\prime}$. However repeated requests have been recorded in geometry discussion forums for an explanation of the particular, seemingly arbitrary, choice of angles made at the beginning of these proofs. Of course the reasoning of the choice is not necessary for their validity. But the readers unfulfilling understanding may have encouraged the mathematical folklore the use of words "mystery", "magic" or "miracle" for referring to Morley's theorem. This is not justifiable as there is nothing mathematically extraordinary related to the theorem.

The presented proofs for showing that the interior, the central and the exterior Morley triangles are equilaterals use the classical Analysis and Synthesis method. They exploit the inherent symmetries of the problem and characterized by their uniform structure, logical transparency, remarkable shortness and the distinct aesthetics of the Euclidean geometry. The Synthesis part follows the previous method scheme. But it is empowered by two simple observations, supplying necessary and sufficient conditions for a point to be the incenter or one of the excenters of a given triangle. Even though they are almost trivial have a subtlety that enables to confront the messy complexity of the triangle trisectors by enforcing clean simplicity and create proofs by harnessing the power of the triangle angle bisector theorem. In addition these proofs reveal fundamental properties of the Morley equilaterals stated as Corollaries. Besides their extensive use for showing Morley triangles as not equilaterals, their fertility is demonstrated by proving the following: (1) The two sides extensions of the inner Morley equilateral meet the corresponding inner trisectors at two points which with the two sides common vertex form an equilateral. (2) The sides of Morley equilaterals are collinear or parallel. (3) In any triangle the exterior trisectors of its angles, proximal to the three sides respectively, meet at the vertices of an equilateral, if and only if, the interior trisectors of an angle and the exterior trisectors of the other two angles, proximal the three sides respectively, meet at the vertices of an equilateral. 
In short, this work advocates that for Morley's observation a natural theoretical setting is Euclidean geometry.

\section{Notation and Counting of all Morley triangles}

In a Morley triangle of $\triangle A B C$ each vertex is the intersection of two trisectors, each of which is either proximal or distal to a side of $\triangle A B C$. Hence a vertex is called proximal, distal or mix with respect to the triangle side it belongs in the case the trisectors are both proximal, both distal, or one proximal and one distal to the side, respectively.

So we may denote a proximal, distal or mix vertex with respect to a side by using as superscripts $p, d$ or $*$ to the letter of the corresponding angle of $\triangle A B C$ opposite to the side.

Thus $A^{p}, A^{d}$ and $A^{*}$ denote the proximal, distal and mix vertex of a Morley triangle with respect to $\mathrm{BC}$ respectively. In Fig. 3 the notations for all intersections of the interior trisectors of $\triangle A B C$ are showed. Notice that a Morley triangle may have either proximal vertices, or distal vertices, or exactly two mix vertices.

Specifically, $\triangle A^{p} B^{p} C^{p}$ denotes the inner Morley triangle of proximal

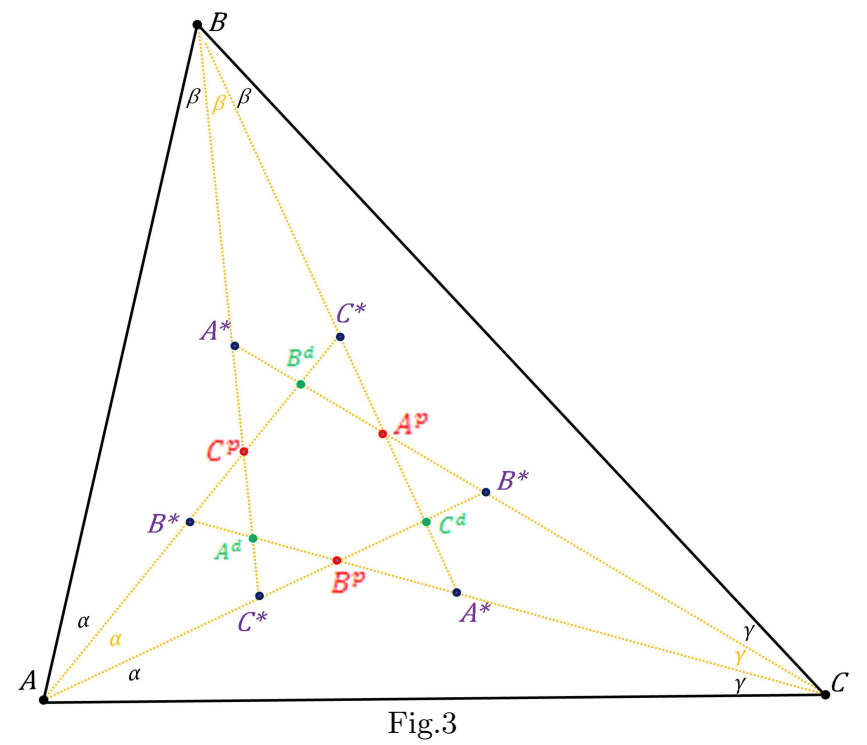
vertices, which is the inner Morley triangle determined by the intersections of proximal to each side trisectors. Also there is just one Morley triangle with distal vertices which is denoted by $\triangle A^{d} B^{d} C^{d}$. In addition there are three Morley triangles with one vertex proximal and two vertices mix. They are denoted by $\triangle A^{p} B^{*} C^{*}, \triangle B^{p} C^{*} A^{*}$ and $\triangle C^{p} A^{*} B^{*}$

Moreover there are three more Morley triangles with one vertex distal and two vertices mix. They are denoted by $\triangle A^{d} B^{*} C^{*}, \triangle B^{d} C^{*} A^{*}$ and $\triangle C^{d} A^{*} B^{*}$. Notice that a proximal or a distal vertex is uniquely determined but a mix vertex is not as there are two such denoted by the same letter. However in a Morley triangle with a pair of mix vertices, given its proximal or distal vertex, the mix vertices are uniquely specified due to the choice restrictions in pairing trisectors for the second and then for the third vertex. Hence there are 8 interior Morley triangles formed by the trisectors of the interior angles of $\triangle A B C$.

Similarly the trisectors of the exterior angles of $\triangle A B C$ form Morley triangles. These are 
denoted by $\triangle A_{A}^{p} B_{B}^{p} C_{C}^{p}$, for the Morley triangle of proximal vertices, $\triangle A_{A}^{d} B_{B}^{d} C_{C}^{d}$, for the Morley triangle of distal vertices, $\triangle A_{A}^{p} B_{B}^{*} C_{C}^{*}, \triangle B_{B}^{p} C_{C}^{*} A_{A}^{*}$ and $\triangle C_{C}^{p} A_{A}^{*} B_{B}^{*}$, for the Morley triangles with one proximal and two mix vertices, $\triangle A_{A}^{d} B_{B}^{*} C_{C}^{*}, \triangle B_{B}^{d} C_{C}^{*} A_{A}^{*}$ and $\triangle C_{C}^{d} A_{A}^{*} B_{B}^{*}$ for the Morley triangles of one distal and two mix vertices. In this notation we use subscripts in order to distinguish a vertex determined by the interior trisectors from the vertex of the same type determined by the exterior trisectors. Hence, in general, there are 8 Morley triangles formed by the trisectors of the exterior angles of $\triangle A B C$. Their vertices are in the exterior of $\triangle A B C$ and due to their rather central location with respect to $\triangle A B C$ are called central Morley triangles.

There are two more possibilities for the formation of a Morley triangle. One is by combining the trisectors of an interior angle with the trisectors of the other two exterior angles of $\triangle A B C$. Another is by combining the trisectors of two interior angles with the trisectors of the third exterior angle of $\triangle A B C$.

The Morley triangles formed by combining the trisectors of the interior $\angle A$ with the trisectors of the exterior $\angle B$ and $\angle C$ are denoted by $\triangle A_{A}^{p} b_{A}^{p} c_{A}^{p}$, for the Morley triangle of proximal vertices, $\triangle A_{A}^{d} b_{A}^{d} c_{A}^{d}$, for the Morley triangle of distal vertices, $\triangle C_{C}^{p} a_{C}^{*} b_{C}^{*}, \triangle a_{C}^{p} b_{C}^{*} C_{C}^{*}$ and $\triangle b_{C}^{p} C_{C}^{*} a_{C}^{*}$, for the Morley triangles with one proximal and two mix vertices, and $\triangle A_{A}^{d} b_{A}^{*} c_{A}^{*}, \triangle b_{A}^{d} c_{A}^{*} A_{A}^{*}$, $\triangle c_{A}^{d} A_{A}^{*} b_{A}^{*}$ for the Morley triangles with one distal and two mix vertices. The use of a small letter is for denoting the intersection of an interior and an exterior trisector of $\triangle A B C$. The vertices of these 8 triangles formed by the trisectors of the interior $\angle \mathrm{A}$ with the trisectors of the exterior $\angle \mathrm{B}$ and $\angle C$ are in the exterior of $\triangle A B C$ and thus they are called exterior Morley triangles relative to $\angle A$.

Similarly are denoted the Morley triangles relative to $\angle \mathrm{B}$, which are formed by combining the trisectors of the interior angle $\angle \mathrm{B}$ with the trisectors of the exterior $\angle \mathrm{C}$ and $\angle \mathrm{A}$, and also the ones relative to $\angle \mathrm{C}$ formed by combining the trisectors of the interior $\angle \mathrm{C}$ with the trisectors of the exterior $\angle A$ and $\angle B$. Hence, in general, there are 24 exterior Morley triangles determined by the intersections of trisectors of an interior and two exterior angles of $\triangle A B C$.

The Morley triangles formed by combining the trisectors of the interior $\angle \mathrm{B}$ and $\angle \mathrm{C}$ with the trisectors of the exterior $\angle A$ are denoted by $\triangle A^{p} b_{A}^{p} c_{A}^{p}$, for the Morley triangle of proximal vertices, $\triangle A^{d} b_{A}^{d} c_{A}^{d}$, for the Morley triangle of distal vertices, $\triangle A^{p} b_{A}^{*} c_{A}^{*}, \triangle b_{A}^{p} c_{A}^{*} A^{*}, \triangle c_{A}^{p} A^{*} b_{A}^{*}$, for the Morley triangles of one proximal vertex and two mix, $\triangle A^{d} b_{A}^{*} c_{A}^{*}, \triangle b_{A}^{d} c_{A}^{*} A^{*}, \triangle c_{A}^{d} A^{*} b_{A}^{*}$, for the Morley triangles of one distal vertex and two mix. It should be remarked that in this notation the same symbol for the intersection of an interior with an exterior trisector may refer to two different points, an ambiguity which is clarified in a Morley triangle since one of its vertices specifies its type and so the vertex that the symbolism refers. Hence, there are 8 Morley triangles relative to the exterior $\angle A$, which obviously have one vertex inside and two outside $\triangle A B C$. In general, there are 24 Morley triangles of $\triangle A B C$ determined by the intersections of trisectors of one exterior and two interior angles of $\triangle A B C$.

Conclude that in total there are, in general, 64 Morley triangles of $\triangle \mathrm{ABC}$. 


\section{Uniform Proofs for all Morley Equilaterals}

In this part we will prove that five Morley triangles are equilaterals. The proofs are uniform and utilize two basic observations for determining the incenter or an excenter of $\triangle \mathrm{ABC}$ using only one of its bisectors.

Observe that the incenter I is lying on a unique arc passing through two vertices and I. In Fig.4 the unique arc passing through $A, B$ and I is depicted. Obviously

$$
\angle \mathrm{AIB}=180^{\circ}-\frac{1}{2} \angle \mathrm{ABC}=\frac{1}{2} \angle \mathrm{BAC}=90^{\circ}+\frac{1}{2} \angle \mathrm{ACB} .
$$

Thus I may be characterized as the intersection in the interior of $\triangle A B C$ of a bisector with the arc of size $90^{\circ}+$ $\frac{1}{2} \angle A C B$ passing through $A$ and $B$. Clearly an analogous

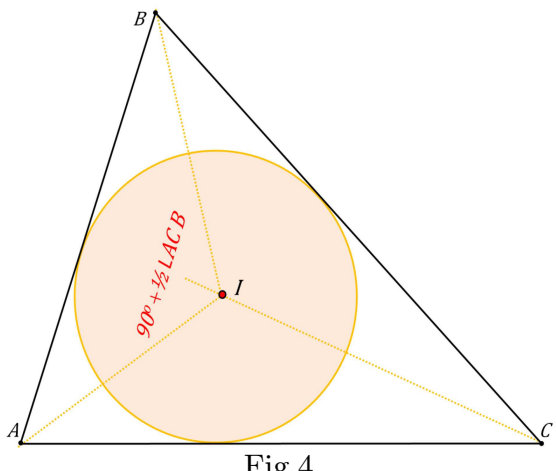
result holds for the other two pairs of vertices of $\triangle A B C$. We refer to this as the Incenter Lemma.

If $\mathrm{I}_{\mathrm{C}}$ is the excenter relative to $\angle \mathrm{C}$ then

$$
\angle A I_{C} B=90^{\circ}-\frac{1}{2} \angle A C B, \angle B I_{C} C=\frac{1}{2} \angle B A C \text { and } \angle C I_{C} A=\frac{1}{2} \angle C B A \text {. }
$$

Thus $I_{C}$ is determined by the intersection in the exterior of $\triangle A B C$ of a bisector, either of the interior $\angle C$ or the exterior $\angle A$ or $\angle B$ with the arc of size $90^{\circ}-\frac{1}{2} \angle A C B$ passing through $A$ and $B$, or with the arc passing through $B$ and $C$ of size $\frac{1}{2} \angle B A C$, or with the arc of size $\frac{1}{2} \angle C B A$ passing through $C$ and $A$. Evidently analogous results hold for the other two excenters $I_{A}$ and $I_{B}$. We refer to this as the Excenter Lemma.

Theorem 1. In any triangle the interior trisectors of its angles, proximal to the sides, meet at the vertices of an equilateral.

\section{Proof.}

Analysis: Let $\triangle A B C$ be a triangle with $\angle A=$ $\overline{3 \alpha, \angle \mathrm{B}}=3 \beta$ and $\angle \mathrm{C}=3 \gamma$, where $\alpha+\beta+\gamma=60^{\circ}$. Suppose that $\triangle A^{p} B^{p} C^{p}$ is equilateral, where $A^{p}$, $\mathrm{B}^{\mathrm{p}}$ and $\mathrm{C}^{\mathrm{p}}$ are the intersections of the trisectors proximal to the sides $B C, C A$ and $A B$ respectively. The aim of this step is to calculate the angles formed by the sides of $\triangle A^{p} B^{p} C^{p}$ and the trisectors of $\triangle A B C$. See Fig. 5 .

Let $C^{d}$ be the intersection of $A B^{p}$ and $B A^{p}$. Since $A C^{p}$ and $B C^{p}$ are angle bisectors in $\triangle A C^{d} B, C^{p}$ is the incenter.

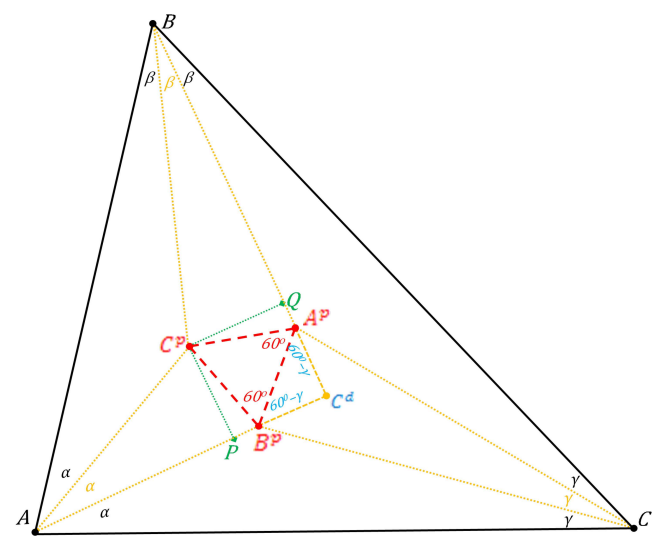

Fig.5 
Let $\mathrm{P}$ and $\mathrm{Q}$ be the orthogonal projections of $\mathrm{C}^{\mathrm{p}}$ on $A C^{\mathrm{d}}$ and $B C^{\mathrm{d}}$ respectively. Thus $\mathrm{C}^{\mathrm{P}} \mathrm{P}=$ $C^{p} \mathrm{Q}$ and $C^{d} \mathrm{P}=C^{d} Q$. But so $\triangle C^{p} P^{p}=\triangle C^{p} Q^{p}$ as right triangles having two pairs of sides equal. Hence $B^{p} P=A^{p} Q$. Then $C^{d} A^{p}=C^{d} B^{p}$ and so $\triangle A^{p} C^{d} B^{p}$ is isosceles. Now from $\triangle A C^{d} B$ we have $\angle A C^{d} B=180^{\circ}-(2 \alpha+2 \beta)=60^{\circ}+2 \gamma$. Therefore $\angle C^{d} B^{p} A^{p}=\angle C^{d} A^{p} B^{p}=$ $\frac{1}{2}\left[180^{\circ}-\left(60^{\circ}+2 \gamma\right)\right]=60^{\circ}-\gamma$. Consequently

$$
\angle C^{p} B^{p} A=\angle C^{p} A^{p} B=180^{\circ}-60^{\circ}-\left(60^{\circ}-\gamma\right)=60^{\circ}+\gamma=\gamma^{+} .
$$

Let $A^{d}$ be the intersection of $B C^{p}$ and $C B^{p}$. Also let $B^{d}$ be the intersection of $C A^{p}$ and $A C^{p}$. Then from $\triangle B A^{d} C$ and $\triangle C B^{d} A$ find similarly

$$
\angle A^{p} C^{p} B=\angle A^{p} B^{p} C=\alpha^{+} \text {and } \angle B^{p} A^{p} C=\angle B^{p} C^{p} A=\beta^{+} .
$$

Synthesis: Suppose that a triangle is given and assume that its angles are trisected and equal to $3 \alpha, 3 \beta$ and $3 \gamma$, respectively, where $\alpha+\beta+\gamma=60^{\circ}$. Then around an equilateral $\triangle A^{p} B^{p} C^{p}$ will construct $\triangle A B C$ with angles $3 \alpha, 3 \beta$ and $3 \gamma$ so that $A^{p}, B^{p}$ and $C^{p}$ will be the intersections of the proximal to the sides interior trisectors.

On the side $B^{p} C^{p}$ erect $\triangle B^{p} A C^{p}$ with adjacent angles $\gamma^{+}=\gamma+60^{\circ}$ and $\beta^{+}=\beta+$ $60^{\circ}$.

Similarly, erect $\triangle C^{p} B A^{p}$ and $\triangle A^{p} C B^{p}$ on the sides $C^{p} A^{p}$ and $A^{p} B^{p}$ respectively with corresponding angles as shown in Fig.6, which were found in the Analysis step.

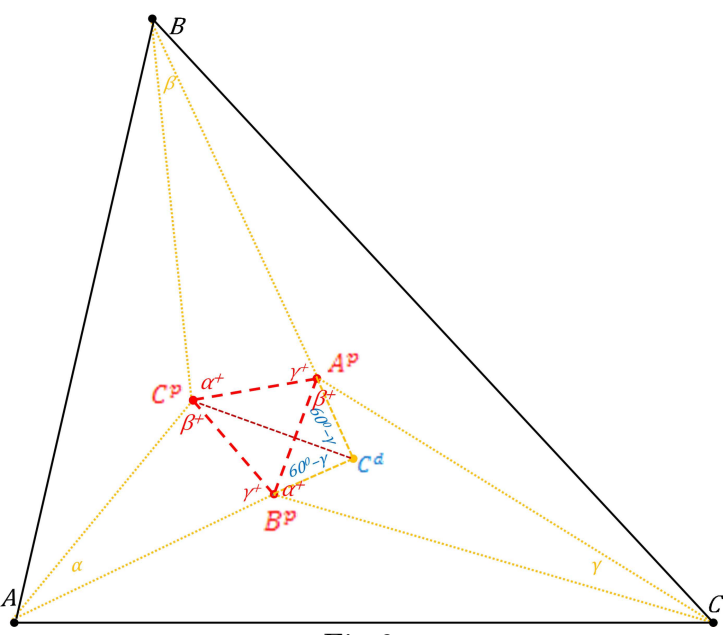

Fig.6

Let $C^{d}$ be the intersection $A B^{p}$ and $B A^{p}$. Notice that $\triangle A^{p} C^{d} B^{p}$ is isosceles as two of its angles are $180^{\circ}-60^{\circ}-\gamma^{+}=60^{\circ}-\gamma$.

Thus

$$
C^{d} A^{p}=C^{d} B^{p}(1) \text { and } \angle A^{p} C^{d} B^{p}=180^{\circ}-2\left(60^{\circ}-\gamma\right)=60^{\circ}+2 \gamma(2)
$$

Since $\triangle A^{p} B^{p} C^{p}$ has been taken equilateral, $C^{p} A^{p}=C^{p} B^{p}$. Combine this with (1) and infer that $C^{p}$ is on the $A^{p} B^{p}$ bisector and so on the $\angle A C^{d} B$ bisector. Moreover from $(2) \angle A C^{p} B=$ $360^{\circ}-\left(\alpha^{+}+60^{\circ}+\beta^{+}\right)=180^{\circ}-(\alpha+\beta)=90^{\circ}+\frac{1}{2}\left(60^{\circ}+2 \gamma\right)=90^{\circ}+\frac{1}{2} \angle A^{p} C^{d} B^{p}=90^{\circ}+\frac{1}{2} \angle A C^{d} B$.

Hence, by the Incenter Lemma, $C^{p}$ is the incenter of $\triangle A C^{d} B$.

Similarly it is shown that $A^{p}$ and $B^{p}$ are the incenters of $\triangle B A^{d} C$ and $\triangle C B^{d} A$, respectively, where $A^{d}$ is the intersection of $B C^{p}$ and $C B^{p}$, while $B^{d}$ is the intersection of $C A^{p}$ and $A C^{p}$. Thus $\angle C^{p} A B=\angle C^{p} A B^{p}=\angle C A B^{p}$ and so $A B^{p}, A C^{p}$ are trisectors of $\angle A$. 
Also the choice of angles in the construction of $\triangle B^{\mathfrak{p}} A C^{p}$ implies $\angle C^{p} A B^{p}=\alpha$. Hence $\angle A=3 \alpha$. Likewise infer that $B C^{p}, B A^{p}$ are trisectors of $\angle B$ with $\angle B=3 \beta$ and $C A^{p}, C^{p}$ are trisectors of $\angle \mathrm{C}$ with $\angle \mathrm{C}=3 \gamma$.

Corollary 1. a) The angles between the trisectors of $\triangle A B C$ and the sides of its inner Morley equilateral $\triangle A^{p} B^{p} C^{p}$ are: $\angle A^{p} B^{p} C=\angle A^{p} C^{p} B=\alpha^{+}, \angle B^{p} C^{p} A=\angle B^{p} A^{p} C=\beta^{+}$, $\angle C^{p} A^{p} B=\angle C^{p} B^{p} A=\gamma^{+}$.

b) The heights of the equilateral $\triangle \mathrm{A}^{p} \mathrm{~B}^{p} C^{p}$ are: $\mathrm{A}^{p} \mathrm{~A}^{\mathrm{d}}, \mathrm{B}^{p} \mathrm{~B}^{\mathrm{d}}$ and $\mathrm{C}^{p} \mathrm{C}^{\mathrm{d}}$.

Theorem 2. In any triangle the exterior trisectors of its angles, proximal to the sides, meet at the vertices of an equilateral.

\section{Proof.}

Analysis: Let $\triangle A B C$ be a triangle with $\angle A=3 \alpha, \angle B=3 \beta$ and $\angle C=3 \gamma$, where $\alpha+\beta+\gamma=$ $60^{\circ}$. Let $A_{A}^{p}, B_{B}^{p}$ and $C_{C}^{p}$ be the intersections of the exterior trisectors proximal to the sides $B C$, $C A$ and $A B$ respectively. Suppose $\triangle A_{A}^{p} B_{B}^{p} C_{C}^{p}$ is equilateral. The aim of this step is to calculate the angles formed by the sides of $\triangle A_{A}^{p} B_{B}^{p} C_{C}^{p}$ and the exterior trisectors of $\triangle A B C$.

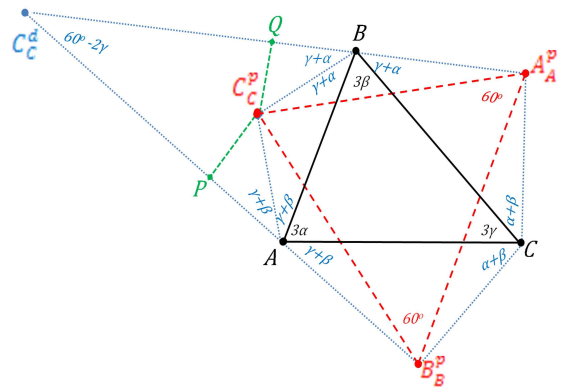

Fig.7a $\left(\gamma<30^{\circ}\right)$

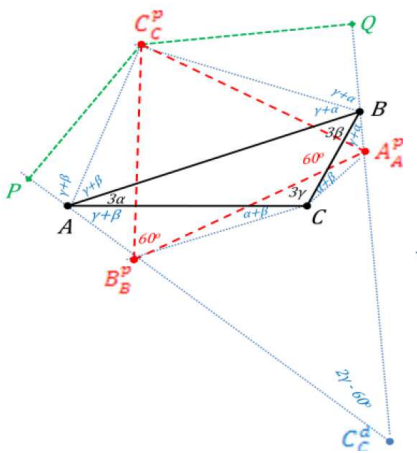

Fig. $7 \mathrm{~b}\left(\gamma>30^{\circ}\right)$

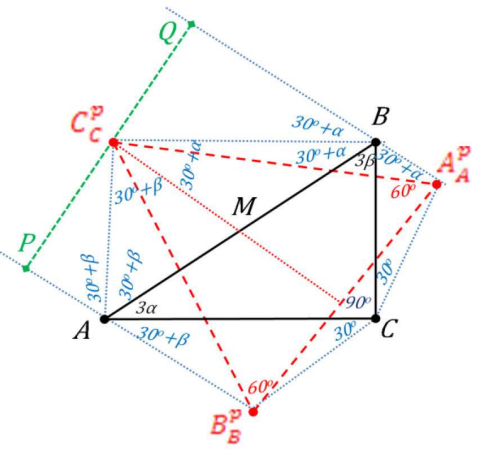

Fig. $7 \mathrm{c}\left(\gamma=30^{\circ}\right)$

Let $\mathrm{P}$ and $\mathrm{Q}$ be the orthogonal projections of $\mathrm{C}_{\mathrm{C}}^{\mathrm{p}}$ on $A B_{B}^{\mathrm{p}}$ and $\mathrm{B} \mathrm{A}_{\mathrm{A}}^{\mathrm{p}}$ respectively.

Notice that $A B_{B}^{p}$ and $B A_{A}^{p}$ may intersect each other or be parallel since

$$
\angle \mathrm{PAB}+\angle \mathrm{QBA}=2(\beta+\gamma)+2(\gamma+\alpha)=120^{\circ}+2 \gamma .
$$

If $A B_{B}^{p}$ and $B A_{A}^{p}$ intersect each other let $C_{C}^{d}$ be their intersection. Next consider all possible cases.

$\triangleright$ If $\gamma<30^{\circ}$ then

$C_{C}^{d}$ and $C_{C}^{p}$ are at the same side of $A B$. In $\triangle A C_{C}^{d} B, A C_{C}^{p}$ and $B C_{C}^{p}$ are interior angle bisectors and so $C_{C}^{p}$ is the incenter, while it is calculated $\angle A C_{C}^{d} B=60^{\circ}-2 \gamma$. Fig.7a. 
$\triangleright$ If $\gamma>30^{\circ}$ then

$C_{C}^{d}$ and $C_{C}^{p}$ are on different sides of $A B$. In $\triangle A C_{C}^{d} B, A C_{C}^{p}$ and $B C_{C}^{p}$ are exterior angle bisectors and so $C_{C}^{p}$ is the excenter relative to $\angle C_{C}^{d}$, while it is calculated $\angle A C_{C}^{d} B=2 \gamma-60^{\circ}$. Fig. $7 \mathrm{~b}$.

Hence in both the above cases $\left(\gamma \neq 30^{\circ}\right)$ it holds $C_{C}^{p} P=C_{C}^{p} Q$. Thus $\triangle C_{C}^{p} P B_{B}^{p}=\triangle C_{C}^{p} Q A_{A}^{p}$, as right triangles having two pairs of sides equal. Consequently $\angle C_{C}^{p} B_{B}^{p} P=\angle C_{C}^{p} A_{A}^{p} Q$ and so $\triangle A_{A}^{p} C_{C}^{d} B_{B}^{p}$ is isosceles. Thus:

$\diamond$ If $\gamma>30^{\circ}$ then

$$
\angle C_{C}^{p} B_{B}^{p} P=\angle C_{C}^{p} A_{A}^{p} Q=\frac{1}{2}\left[180^{\circ}-\angle A_{A}^{p} C_{C}^{d} B_{B}^{p}\right]-60^{\circ}=\frac{1}{2}\left[180^{\circ}-\left(60^{\circ}-2 \gamma\right)\right]-60^{\circ}=\gamma .
$$

$\diamond$ If $\gamma<30^{\circ}$ then

$\angle C_{C}^{p} B_{B}^{p} P=\angle C_{C}^{p} A_{A}^{p} Q=180^{\circ}-60^{\circ}-\frac{1}{2}\left[180^{\circ}-\angle A_{A}^{p} C_{C}^{d} B_{B}^{p}\right]=180^{\circ}-60^{\circ} \frac{1}{2}\left[180^{\circ}-\left(2 \gamma-60^{\circ}\right)\right]=\gamma$.

Deduce that for $\gamma \neq 30^{\circ}$ it holds $\angle C_{C}^{p} B_{B}^{p} A=\angle C_{C}^{p} A_{A}^{p} B=\gamma$.

$\triangleright$ If $\gamma=30^{\circ}$ then $\alpha+\beta=30^{\circ}$ and $A B_{B}^{p} / / B A_{A}^{p}$. Fig.7c.

Notice $\angle A C_{C}^{p} B=180^{\circ}-\left(30^{\circ}+\beta\right)-\left(30^{\circ}+\alpha\right)$ and so $\angle A C_{C}^{p} B=90^{\circ}$.

Let $M$ be the midpoint of $A B$. Since $\triangle A C_{C}^{p} B$ is right triangle, $C_{C}^{p} M=M A=M B$. Then $C_{C}^{p} M=$ $M A$ gives $\angle C_{C}^{p} A M=\angle M C_{C}^{p} A$. But $A C_{C}^{p}$ is the $\angle P A B$ bisector and thus $\angle C_{C}^{p} A M=\angle C_{C}^{p} A P$. Hence $\angle M C_{C}^{p} A=\angle C_{C}^{p} A P$ and so $B_{B}^{p} A / / C_{C}^{p} M / / A_{A}^{p} B$.

Since $M A=M B, C_{C}^{p} M$ bisects $A_{A}^{p} B_{B}^{p}$ and so $C_{C}^{p} M \perp A_{A}^{p} B_{B}^{p}$, as $\triangle A_{A}^{p} B_{B}^{p} C_{C}^{p}$ is equilateral. Then $A B_{B}^{p}, B A_{A}^{p} \perp A_{A}^{p} B_{B}^{p}$. Therefore $\angle A B_{B}^{p} A_{A}^{p}=90^{\circ}$ and given that $\angle C_{C}^{p} B_{B}^{p} A_{A}^{p}=60^{\circ}$ infer $\angle C_{C}^{p} B_{B}^{p} A=30^{\circ}$. Similarly infer $\angle C_{C}^{p} A_{A}^{p} B=30^{\circ}$.

Deduce that for $\gamma=30^{\circ}$ it holds $\angle C_{C}^{p} B_{B}^{p} A=\angle C_{C}^{p} A_{A}^{p} B=\gamma$.

- Conclude that for any value of $\gamma$ it holds $\angle C_{C}^{p} B_{B}^{p} A=\angle C_{C}^{p} A_{A}^{p} B=\gamma$.

Then from $\triangle C_{C}^{p} A B_{B}^{p}$ and $\triangle C_{C}^{p} B A_{A}^{p}$ deduce $\angle B_{B}^{p} C_{C}^{p} A=\beta$ and $\angle A_{A}^{p} C_{C}^{p} B=\alpha$ respectively. Also from $\triangle B A_{A}^{p} C$ and $\triangle C B_{B}^{p} A$ infer $\angle B_{B}^{p} A_{A}^{p} C=\beta$ and $\angle A_{A}^{p} B_{B}^{p} C=\alpha$.

Synthesis: Let a triangle be given in which its angles are equal to $3 \alpha, 3 \beta$ and $3 \gamma$ respectively, where $\alpha+\beta+\gamma=60^{\circ}$. Then around an equilateral, which is denoted by $\triangle A_{A}^{p} B_{B}^{p} C_{C}^{p}$, will construct a $\triangle A B C$ with angles $3 \alpha, 3 \beta$ and $3 \gamma$ so that $A_{A}^{p}, B_{B}^{p}$ and $C_{C}^{p}$ will be the meeting points of the exterior angle trisectors proximal to the sides of $\triangle A B C$. On the side $B_{B}^{p} C_{C}^{p}$ erect $\triangle B_{B}^{p} A C_{C}^{p}$ with adjacent angles $\gamma$ and $\beta$ which were calculated in the Analysis step. Similarly, erect $\triangle C_{C}^{p} B A_{A}^{p}$ and $\triangle A_{A}^{p} C_{B}^{p}$ on the sides $C_{C}^{p} A_{A}^{p}$ and $A_{A}^{p} B_{B}^{p}$ with corresponding angles as they are depicted in Fig.8. Hence $\triangle A B C$ has been determined. So it remains to be proved that the resulting $\triangle A B C$ has angles $3 \alpha, 3 \beta$ and $3 \gamma$ respectively and the erected sides are the trisectors of its exterior angles.

Let $\mathrm{P}$ and $\mathrm{Q}$ be the orthogonal projections of $C_{C}^{p}$ on the extensions of $A B_{B}^{p}$ and $B A_{A}^{p}$ respectively. Next consider all cases regarding $A B_{B}^{p}$ and $B A_{A}^{p}$. 


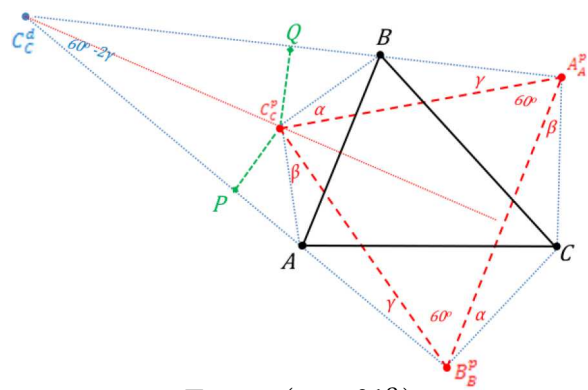

Fig. $8 \mathrm{a}\left(\gamma<30^{\circ}\right)$

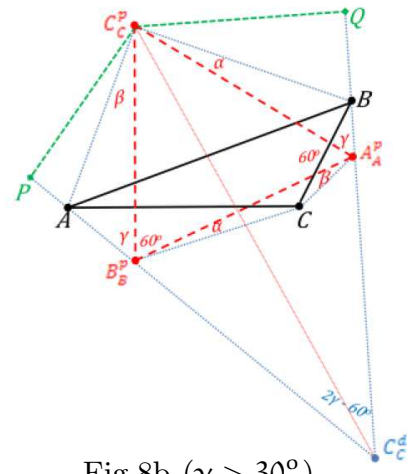

Fig. $8 \mathrm{~b}\left(\gamma>30^{\circ}\right)$

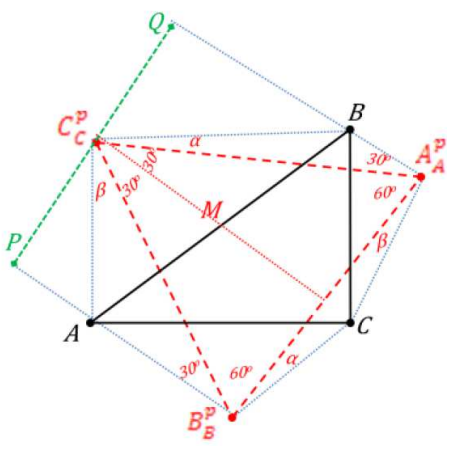

Fig. $8 \mathrm{c}\left(\gamma=30^{\circ}\right)$

$\triangleright$ Assume $\gamma \neq 30^{\circ}$. Set $s=\left(30^{\circ}-\gamma\right) /\left|30^{\circ}-\gamma\right|$ and let $C_{C}^{d}$ be the meeting point of $A B_{B}^{p}$ and $A_{A}^{p} B$. The choice of angles in the erection of $\triangle B_{B}^{p} A C_{C}^{p}$ and $\triangle C_{C}^{p} B A_{A}^{p}$ implies:

$C_{C}^{p}$ is lying on the $\angle A_{A}^{p} C_{C}^{d} B_{B}^{p}$ bisector (1) and $\angle A C_{C}^{p} B=90^{\circ}+\frac{1}{2} s \angle A C_{C}^{d} B(2)$

To verify (1) notice that $\triangle A_{A}^{p} C_{C}^{d} B_{B}^{p}$ is isosceles, as two of its angles are by construction either $60^{\circ}+\gamma\left(\gamma<30^{\circ}\right)$ or $120^{\circ}-\gamma\left(\gamma>30^{\circ}\right)$. But $\triangle A_{A}^{p} B_{B}^{p} C_{C}^{p}$ is assumed equilateral and so $C_{C}^{p} A_{A}^{p}=C_{C}^{p} B_{B}^{p}$. Thus $C_{C}^{d} C_{C}^{p}$ bisects side $A_{A}^{p} B_{B}^{p}$ of the isosceles $\triangle A_{A}^{p} C_{C}^{d} B_{B}^{p}$ and so $C_{C}^{p}$ is lying on the $\angle A_{A}^{p} C_{C}^{d} B_{B}^{p}$ bisector.

To verify (2) notice that in the isosceles $\triangle A_{A}^{p} C_{C}^{d} B_{B}^{p}$ either $\angle A C_{C}^{d} B=180^{\circ}-2\left(60^{\circ}+\gamma\right)-$ $60^{\circ}-2 \gamma\left(\gamma<30^{\circ}\right)$ or $\angle A C_{C}^{\mathrm{d}} B=180^{\circ}-2\left(60^{\circ}+\gamma\right)=2 \gamma-60^{\circ}\left(\gamma>30^{\circ}\right)$. Thus $\angle A C_{C}^{\mathrm{d}} \mathrm{B}=s\left(60^{\circ}-2 \gamma\right)$. Hence $\quad \angle A C_{C}^{p} B=\beta+60^{\circ}+\beta=60^{\circ}+\left(60^{\circ}-\gamma\right)=90^{\circ}+\frac{1}{2} s\left(60^{\circ}-2 \gamma\right)=90^{\circ}+s \angle A C_{C}^{d} B$.

Therefore from (1) and (2), by the Incenter Lemma $\left(\gamma<30^{\circ}, s=1\right)$ or the Excenter Lemma $\left(\gamma>30^{\circ}, s=-1\right), C_{C}^{p}$ is the incenter or the excenter of $\triangle A C_{C}^{d} B$ respectively.

Thus $A C_{C}^{p}$ and $B C_{C}^{p}$ are bisectors (interior or exterior) in $\triangle A C_{C}^{d} B$. So, using $\triangle C_{C}^{p} A B_{B}^{p}$ and $\triangle C_{C}^{p} B A_{A}^{p}$, deduce $\angle C_{C}^{p} A B=\angle C_{C}^{p} A P=\gamma+\beta$ and $\angle C_{C}^{p} B A=\angle C_{C}^{p} B Q=\gamma+\beta$. Consequently $\angle C_{C}^{p} A B=\gamma+\beta$ and $\angle C_{C}^{p} B A=\gamma+\beta$ while $A C_{C}^{p}$ and $B C_{C}^{p}$ bisect the angles formed by $A B$ and the extensions of $A B_{B}^{p}$ and $B A_{A}^{p}$ respectively.

$\triangleright$ Assume $\gamma=30^{\circ}$. Then $\alpha+\beta=30^{\circ}$. Notice $A B_{B}^{p}, B A_{A}^{p} \perp A_{A}^{p} B_{B}^{p}$ and so $A_{A}^{p} B / / B_{B}^{p} A$. Also $\angle A C_{C}^{p} B=\beta+60^{\circ}+\alpha=90^{\circ}$ and so $\triangle A C_{C}^{p} B$ is right triangle. Let $M$ be the midpoint of $A B$. Hence $C_{C}^{p} M=M A=M A$. But $C_{C}^{p} M=M A$ implies $\angle C_{C}^{p} A M=\angle M C_{C}^{p} A$. Since $\angle C_{C}^{p} A M=\angle C_{C}^{p} A P$, $\angle C_{C}^{p} A M=\angle A C_{C}^{p} M$. Consequently $C_{C}^{p} M / / A P$. Thus $A_{A}^{p} B / / C_{C}^{p} M / / B_{B}^{p} A$ and since $M A=M A$, $C_{C}^{p} M$ passes through the midpoint of $A_{A}^{p} B_{B}^{p}$. As a result $C_{C}^{p} M$ is a height of the equilateral $\triangle A_{A}^{p} B_{B}^{p} C_{C}^{p}$ and so $\angle A_{A}^{p} C_{C}^{p} M=\angle B_{B}^{p} C_{C}^{p} M=30^{\circ}$. Therefore

$$
\angle C_{C}^{p} A B=\angle M C_{C}^{p} A=\angle M C_{C}^{p} B_{B}^{p}=\angle B_{B}^{p} C_{C}^{p} A=30^{\circ}+\beta=\gamma+\beta .
$$

Similarly it is shown $\angle C_{C}^{p} B A=30^{\circ}+\alpha=\gamma+\alpha$.

Also $A_{A}^{p} B / / C_{C}^{p} M / / B_{B}^{p} A$ implies $\angle C_{C}^{p} A P=\angle A C_{C}^{p} M$ and $\angle Q B C_{C}^{p}=\angle B C_{C}^{p} M$. So

$$
\angle C_{C}^{p} A P=\angle C_{C}^{p} A B \text { and } \angle C_{C}^{p} B Q=\angle C_{C}^{p} B A \text {. }
$$


- Conclude for any $\gamma$ it holds $\angle C_{C}^{p} A B=\gamma+\beta$ and $\angle C_{C}^{p} B A=\gamma+\alpha$, while $A C_{C}^{p}$ and $B C_{C}^{p}$ bisect the angles formed by $A B$ and the extensions of $A B_{B}^{p}$ and $B A_{A}^{p}$ respectively.

The rest cases are treated similarly. Considering $B C_{C}^{p}$ and $C B_{B}^{p}$ it shown that $\angle A_{A}^{p} B C=\alpha+\gamma$ and $\angle A_{A}^{p} C B=\alpha+\beta$ while $B A_{A}^{p}$ and $C A_{A}^{p}$ bisect the angles formed by $B C$ and the extensions of $B C_{C}^{p}$ and $C B_{B}^{p}$ respectively, and eventually considering $C A_{A}^{p}$ and $A C_{C}^{p}$ it is proved that $\angle B_{B}^{p} C A=\beta+\alpha$ and $\angle B_{B}^{p} A C=\beta+\gamma$ while $C B_{B}^{p}$ and $A B_{B}^{p}$ bisect the angles formed by $A C$ and the extensions of $A C_{C}^{p}$ and $C A_{A}^{p}$ respectively. Conclude that $A B_{B}^{p}$ bisects the angle between $A C$ and the extension of $A C_{C}^{p}$, while $A C_{C}^{p}$ bisects the angle between $A B$ and the extension of $A B_{B}^{p}$. Thus $A B_{B}^{p}$ and $A C_{C}^{p}$ are trisectors of the exterior $\angle A$.

Also $\angle C_{C}^{p} A B=\angle B_{B}^{p} A C=\gamma+\beta$. Hence $\angle A=\frac{1}{2}\left[360^{\circ}-6(\beta+\gamma)\right]=180^{\circ}-3(\beta+\gamma)=3 \alpha$. Similarly it is shown that $B C_{C}^{p}, B A_{A}^{p}$ are trisectors of the exterior $\angle B$ with $\angle B=3 \beta$ and $C A_{A}^{p}$, $\mathrm{CB}_{\mathrm{B}}^{\mathrm{p}}$ are trisectors of the exterior $\angle \mathrm{C}$ with $\angle \mathrm{C}=3 \gamma$.

Corollary 2. a) The angles between the exterior trisectors of $\triangle A B C$ and the sides of its central Morley equilateral $\triangle A_{A}^{p} B_{B}^{p} C_{C}^{p}$ are: $\angle A_{A}^{p} B_{B}^{p} C=\angle A_{A}^{p} C_{C}^{p} B=\alpha, \angle B_{B}^{p} C_{C}^{p} A=\angle B_{B}^{p} A_{A}^{p} C=\beta$, $\angle \mathrm{C}_{\mathrm{C}}^{\mathrm{p}} \mathrm{A}_{\mathrm{A}}^{\mathrm{p}} \mathrm{B}=\angle \mathrm{C}_{\mathrm{C}}^{\mathrm{p}} \mathrm{B}_{\mathrm{B}}^{\mathrm{p}} \mathrm{A}=\gamma$.

b) The heights of the equilateral $\triangle A_{A}^{p} B_{B}^{p} C_{C}^{p}$ are: $A_{A}^{p} A_{A}^{d}, B_{B}^{p} B_{B}^{d}$ and $C_{C}^{p} C_{C}^{d}$.

Theorem 3. In any triangle the interior trisectors of an angle and the exterior trisectors of the other two angles, proximal the three sides respectively, meet at the vertices of an equilateral.

\section{Proof.}

Analysis: Let $\triangle \mathrm{ABC}$ be a triangle with $\angle \mathrm{A}=3 \alpha, \angle \mathrm{B}=3 \beta$ and $\angle \mathrm{C}=3 \gamma$, where $\alpha+\beta+\gamma=60^{\circ}$. Let $C_{C}^{p}$ be the intersection of the exterior trisectors of $\angle B$ and $\angle C$, proximal to $A B$, while $a_{C}^{p}$ and $b_{C}^{p}$ are the intersections of the interior with the exterior trisectors proximal to $B C$ and $C A$ respectively. Suppose that $\triangle a_{C}^{p} C_{C}^{p} b_{C}^{p}$ is equilateral. The aim of this step is to calculate the angles between the sides of $\triangle \mathrm{a}_{\mathrm{C}}^{\mathrm{p}} \mathrm{C}_{\mathrm{C}}^{\mathrm{p}} \mathrm{b}_{\mathrm{C}}^{\mathrm{p}}$ and the interior trisectors of $\angle \mathrm{C}$ and also the exterior trisectors of $\angle A$ and $\angle B$.

Let $\mathrm{P}$ and $\mathrm{Q}$ be the orthogonal projections of $\mathrm{C}_{\mathrm{C}}^{\mathrm{p}}$ on $A b_{C}^{\mathrm{p}}$ and $B a_{C}^{p}$, respectively. It was observed in the course of the Analysis Step of Theorem 2 that the trisectors $A b_{C}^{p}$ and $B a_{C}^{p}$ intersect each other iff $\gamma \neq 30^{\circ}$. Recall that if $\gamma \neq 30^{\circ} \mathrm{C}_{\mathrm{C}}^{\mathrm{p}}$ is the incenter $\left(\gamma<30^{\circ}\right)$ or the excenter $\left(\gamma>30^{\circ}\right)$ of $\triangle \mathrm{BC}_{\mathrm{C}}^{\mathrm{d}} \mathrm{C}$ while for $\gamma=30^{\circ} \mathrm{Ab}_{\mathrm{C}}^{\mathrm{p}} / / \mathrm{Ba}_{\mathrm{C}}^{\mathrm{p}}$. But it was shown that in either case it holds $\mathrm{C}_{\mathrm{C}}^{\mathrm{p}} \mathrm{P}=\mathrm{C}_{\mathrm{C}}^{\mathrm{p}} \mathrm{Q}$ and hence $\triangle \mathrm{C}_{\mathrm{C}}^{\mathrm{p}} \mathrm{Pb}_{\mathrm{C}}^{\mathrm{p}}=\triangle \mathrm{C}_{\mathrm{C}}^{\mathrm{p}} \mathrm{Qa}_{\mathrm{C}}^{\mathrm{p}}$, as right triangles having two pairs of sides equal. This implies $\angle A b_{C}^{p} C_{C}^{p}=\angle B a_{C}^{p} C_{C}^{p}(1)$. Consequently:

$\triangleright$ If $\gamma \neq 30^{\circ}$ then $C_{C}^{d}$ is determined. Hence (1) implies $\angle b_{C}^{p} a_{C}^{p} C_{C}^{d}=\angle a_{C}^{p} b_{C}^{p} C_{C}^{d}$. Thus $\triangle \mathrm{a}_{\mathrm{C}}^{\mathrm{p}} \mathrm{C}_{\mathrm{C}}^{\mathrm{d}} \mathrm{b}_{\mathrm{C}}^{\mathrm{p}}$ is isosceles. However from $\triangle A C_{C}^{\mathrm{d}} \mathrm{B}$ it is calculated that 


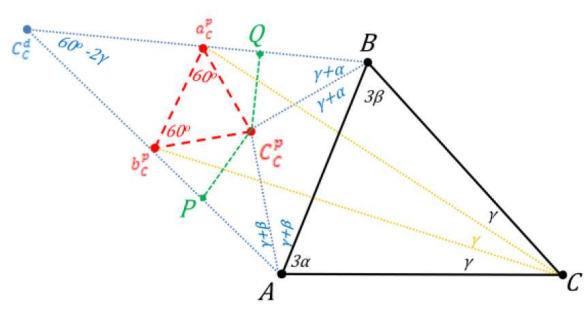

Fig.9a $\left(\gamma<30^{\circ}\right)$

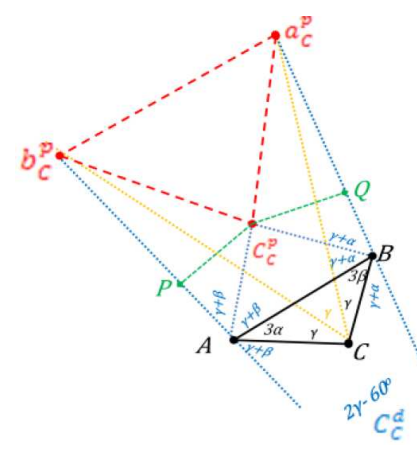

Fig. $9 \mathrm{~b}\left(\gamma>30^{\circ}\right)$

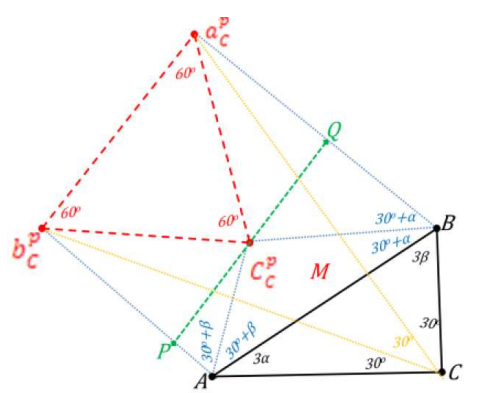

Fig.9c $\left(\gamma=30^{\circ}\right)$

$$
\angle A C_{C}^{\mathrm{d}} \mathrm{B}=60^{\circ}-2 \gamma\left(\gamma<30^{\circ}\right) \text { or } \angle A C_{C}^{\mathrm{d}} \mathrm{B}=2 \gamma-60^{\circ}\left(\gamma>30^{\circ}\right) .
$$

Then we have respectively.

$\diamond$ For $\gamma<30^{\circ}, C_{C}^{d}$ is on the other side of $a_{C}^{p} b_{C}^{p}$ from $A, B$ and

$$
\angle A b_{C}^{p} a_{C}^{p}=\angle B a_{C}^{p} b_{C}^{p}=180^{\circ}-\frac{1}{2}\left[180^{\circ}-\left(60^{\circ}-2 \gamma\right)\right]=120^{\circ}-\gamma .
$$

$\diamond$ For $\gamma>30^{\circ}, C_{C}^{d}$ is on the same side of $a_{C}^{p} b_{C}^{p}$ with $A, B$ and

$$
\angle A b_{C}^{p} a_{C}^{p}=\angle B a_{C}^{p} b_{C}^{p}=\frac{1}{2}\left[180^{\circ}-\left(2 \gamma-60^{\circ}\right)\right]=120^{\circ}-\gamma
$$

In either case $\angle C_{C}^{p} a_{C}^{p} B=\angle C_{C}^{p} b_{C}^{p} A=60^{\circ}-\gamma=\alpha+\beta$.

$\triangleright$ If $\gamma=30^{\circ}$ then $\mathrm{Ab}_{\mathrm{C}}^{\mathrm{p}} / / \mathrm{Ba}_{\mathrm{C}}^{\mathrm{p}}$. Also $\mathrm{C}_{\mathrm{C}}^{\mathrm{p}} \mathrm{P}, \mathrm{C}_{\mathrm{C}}^{\mathrm{p}} \mathrm{Q}$ are collinear and $\alpha+\beta=30^{\circ}$. Thus $\angle \mathrm{a}_{\mathrm{C}}^{\mathrm{p}} \mathrm{C}_{\mathrm{C}}^{\mathrm{p}} \mathrm{b}_{\mathrm{C}}^{\mathrm{p}}=180^{\circ}-\left(30^{\circ}+\beta\right)-\left(30^{\circ}+\alpha\right)=60^{\circ}$ and so by $(1) \angle \mathrm{a}_{\mathrm{C}}^{\mathrm{p}} \mathrm{C}_{\mathrm{C}}^{\mathrm{p}} \mathrm{Q}=\angle \mathrm{b}_{\mathrm{C}}^{\mathrm{p}} \mathrm{C}_{\mathrm{C}}^{\mathrm{p}} \mathrm{P}=$ $\frac{1}{2}\left(180^{\circ}-\angle a_{C}^{p} C_{C}^{p} b_{C}^{p}\right)=\frac{1}{2}\left(180^{\circ}-60^{\circ}\right)=60^{\circ}$. Hence $\angle C_{C}^{p} a_{C}^{p} B=\angle C_{C}^{p} b_{C}^{p} A=30^{\circ}=\alpha+\beta$.

○ In conclusion for any $\gamma$ it holds $\angle A b_{C}^{p} C_{C}^{p}=\angle B a_{C}^{p} C_{C}^{p}=60^{\circ}-\gamma=\alpha+\beta$.

Finally from $\triangle B a_{C}^{p} C$ and $\triangle \mathrm{Cb}_{C}^{p} A$ we find $\angle B a_{C}^{p} C=\alpha$ and $\angle A b_{C}^{p} C=\beta$ respectively, and so $\angle C_{C}^{p} a_{C}^{p} c=\beta$ and $\angle C_{C}^{p} b_{C}^{p} c=\alpha$. Yet from $\triangle b_{C}^{p} A C_{C}^{p}$ and $\triangle C_{C}^{p} B a_{C}^{p}$ calculate that $\angle \mathrm{b}_{\mathrm{C}}^{\mathrm{p}} \mathrm{C}_{\mathrm{C}}^{\mathrm{p}} \mathrm{A}=(\gamma+\alpha)^{+}$and $\angle \mathrm{a}_{\mathrm{C}}^{\mathrm{p}} \mathrm{C}_{\mathrm{C}}^{\mathrm{p}} \mathrm{B}=(\gamma+\beta)^{+}$.

Synthesis: Suppose that a triangle is given with angles equal to $3 \alpha, 3 \beta$ and $3 \gamma$, respectively, where $\alpha+\beta+\gamma=60^{\circ}$. Then from an equilateral, which we denote $\triangle \mathrm{a}_{\mathrm{C}}^{\mathrm{p}} \mathrm{C}_{\mathrm{C}}^{\mathrm{p}} \mathrm{b}_{\mathrm{C}}^{\mathrm{p}}$, will construct a $\triangle A B C$ with angles $3 \alpha, 3 \beta$ and $3 \gamma$ so that the sides of the erected triangles are the proper angle trisectors of the resulting $\triangle A B C$. On the side $a_{C}^{p} b_{C}^{p}$ erect $\triangle a_{C}^{p} C b_{C}^{p}$ with adjacent angles $\beta^{+}=60^{\circ}+\beta$ and $\alpha^{+}=60^{\circ}+\alpha$ so that $C_{C}^{p}$ is inside $\triangle a_{C}^{p} C_{b}^{p}$. Next on the side $b_{C}^{p} C_{C}^{p}$ erect $\triangle \mathrm{b}_{\mathrm{C}}^{\mathrm{p}} \mathrm{A} \mathrm{C}_{\mathrm{C}}^{\mathrm{p}}$ with adjacent angles $\alpha+\beta$ and $(\gamma+\alpha)^{+}$. Finally on the side $C_{\mathrm{C}}^{\mathrm{p}} \mathrm{a}_{\mathrm{C}}^{\mathrm{p}}$ erect $\triangle \mathrm{a}_{\mathrm{C}}^{\mathrm{p}} \mathrm{BC}_{\mathrm{C}}^{\mathrm{p}}$ with adjacent angles $\alpha+\beta$ and $(\gamma+\beta)^{+}$. Thus $\triangle A B C$ has been determined. See Fig.10 for the corresponding value of $\gamma$. So it remains to be proved that the resulting $\triangle A B C$ has angles $3 \alpha, 3 \beta$ and $3 \gamma$, respectively and the erected sides $C a_{C}^{p}, C b_{C}^{p}$ are trisectors of $\angle C$, while $A b_{C}^{p}, A C_{C}^{p}$, and 
$\mathrm{BC}_{\mathrm{C}}^{\mathrm{p}}, \mathrm{Ba}_{\mathrm{C}}^{\mathrm{p}}$ are trisectors of the exterior angles $\angle \mathrm{A}$ and $\angle \mathrm{B}$ respectively.

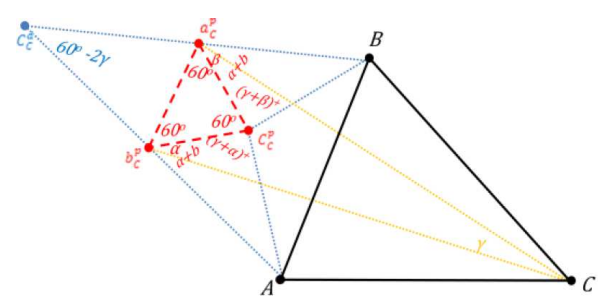

Fig.10a $\left(\gamma<30^{\circ}\right)$

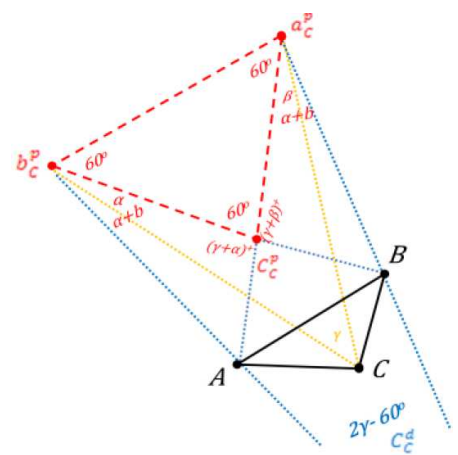

Fig.10b $\left(\gamma>30^{\circ}\right)$

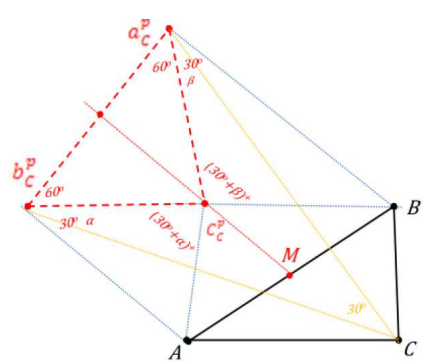

Fig.10c $\left(\gamma=30^{\circ}\right)$

Notice that if either $\gamma<30^{\circ}$ or $\gamma>30^{\circ}$ then $A b_{C}^{p}$ and $B a_{C}^{p}$ intersect each other, while for $\gamma=30^{\circ} A b_{C}^{p} / / \mathrm{Ba}_{\mathrm{C}}^{\mathrm{p}}$.

First we deal with the erected sides $\mathrm{Ca}_{\mathrm{C}}^{\mathrm{p}}$ and $\mathrm{Cb}_{\mathrm{C}}^{\mathrm{p}}$ and prove that they are trisectors of $\angle \mathrm{C}$. We also show that $\angle \mathrm{C}=3 \gamma$. See Fig. 11 .

Let $b_{C}^{d}$ be the intersection of $A C_{C}^{p}$ and $C_{C}^{p}$. Notice the choice of angles in the construction of $\triangle \mathrm{a}_{\mathrm{C}}^{\mathrm{p}} \mathrm{Cb}_{\mathrm{C}}^{\mathrm{p}}$ and $\triangle \mathrm{a}_{\mathrm{C}}^{\mathrm{p}} \mathrm{BC}_{\mathrm{C}}^{\mathrm{p}}$ yields $\angle \mathrm{Ca}_{\mathrm{C}}^{\mathrm{p}} \mathrm{C}_{\mathrm{C}}^{\mathrm{p}}=\beta$ and $\angle \mathrm{a}_{\mathrm{C}}^{\mathrm{p}} \mathrm{C}_{\mathrm{C}}^{\mathrm{p}} \mathrm{b}_{\mathrm{C}}^{\mathrm{d}}=180^{\circ}-(\gamma+\alpha)^{+}-60^{\circ}=\beta$. Hence $\triangle \mathrm{a}_{\mathrm{C}}^{\mathrm{p}} \mathrm{b}_{\mathrm{C}}^{\mathrm{d}} \mathrm{C}_{\mathrm{C}}^{\mathrm{p}}$ is isosceles. Thus $\angle A \mathrm{~b}_{\mathrm{C}}^{\mathrm{d}} \mathrm{C}=2 \beta$ and $\angle A b_{C}^{p} \mathrm{C}=\frac{1}{2} \angle A b_{C}^{d} C$.

Since $\triangle a_{C}^{p} b_{C}^{d} C_{C}^{p}$ is isosceles and from the assumption $\triangle a_{C}^{p} C_{C}^{p} b_{C}^{p}$ is equilateral, infer that $b_{C}^{d} b_{C}^{p}$ bisects $a_{C}^{p} C_{C}^{p}$ and so $b_{C}^{d} b_{C}^{p}$ is the $\angle a_{C}^{p} b_{C}^{d} C_{C}^{p}$ bisector. Hence $b_{C}^{d}$ is lying on the exterior bisector of $\triangle A b_{C}^{d} C$.

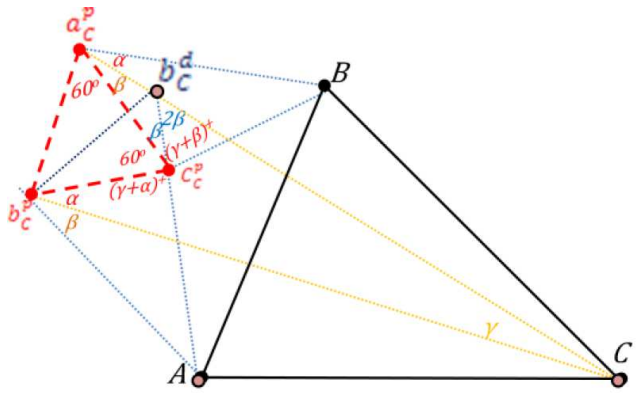

Fig.11 Thus, by the Excenter Lemma, $b_{C}^{p}$ is the excenter of $\triangle A b_{C}^{d} C$ relative to $\angle C$. But so $C_{C}^{p}$ is the $\angle A C a_{C}^{p}$ bisector.

Similarly show that $\mathrm{Ca}_{\mathrm{C}}^{\mathrm{p}}$ is the $\angle \mathrm{BCb} \mathrm{C}_{\mathrm{C}}^{\mathrm{p}}$ bisector.

Therefore $\mathrm{Cb}_{\mathrm{C}}^{\mathrm{p}}$ and $\mathrm{Ca}_{\mathrm{C}}^{\mathrm{p}}$ are trisectors of $\angle \mathrm{C}$. Also

$$
\angle \mathrm{a}_{\mathrm{C}}^{\mathrm{p}} \mathrm{Cb}_{\mathrm{C}}^{\mathrm{p}}=180^{\circ}-\angle \mathrm{Cb}_{\mathrm{C}}^{\mathrm{p}} \mathrm{a}_{\mathrm{C}}^{\mathrm{p}}-\angle \mathrm{Ca}_{\mathrm{C}}^{\mathrm{p}} \mathrm{b}_{\mathrm{C}}^{\mathrm{p}}=180^{\circ}-\alpha^{+}-\beta^{+}=\gamma .
$$

Conclude $\angle \mathrm{C}=3 \gamma$.

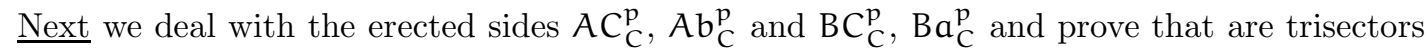
of $\angle A$ and $\angle B$. We also show that $\angle A=3 \alpha$ and $\angle B=3 \beta$. 
$\triangleright$ Assume $\gamma \neq 30^{\circ}$. Set $s=\left(30^{\circ}-\gamma\right) /\left|30^{\circ}-\gamma\right|$ and let $C_{C}^{d}$ be the meeting point of $A b_{C}^{p}$ and $B a_{C}^{p}$. The choice of angles in the erection of $\triangle C_{C}^{p} A b_{C}^{p}$ and $\triangle C_{C}^{p} B a_{C}^{p}$ implies:

$C_{C}^{p}$ is lying on the $\angle A C_{C}^{d} B$ bisector (1) and $\angle A C_{C}^{p} B=90^{\circ}+\frac{1}{2} s \angle A C_{C}^{d} B(2)$

To verify (1) notice that $\triangle a_{A}^{p} C_{C}^{d} b_{C}^{p}$ is isosceles, as two of its angles are either $120^{\circ}-(\alpha+\beta)$ $\left(\gamma<30^{\circ}\right)$ or $60^{\circ}+(\alpha+\beta)\left(\gamma>30^{\circ}\right)$. Using the fact that $\triangle a_{C}^{p} C_{C}^{p} b_{C}^{p}$ is equilateral, infer that $\mathrm{C}_{\mathrm{C}}^{\mathrm{d}} \mathrm{C}_{\mathrm{C}}^{\mathrm{p}}$ bisects $\mathrm{a}_{\mathrm{C}}^{\mathrm{p}} \mathrm{b}_{\mathrm{C}}^{\mathrm{p}}$ and so $\mathrm{C}_{\mathrm{C}}^{\mathrm{p}}$ is lying on the $\angle \mathrm{a}_{\mathrm{C}}^{\mathrm{p}} \mathrm{C}_{\mathrm{C}}^{\mathrm{d}} \mathrm{b}_{\mathrm{C}}^{\mathrm{p}}$ bisector.

To verify (2) notice that in the isosceles $\triangle \mathrm{a}_{\mathrm{C}}^{\mathrm{p}} \mathrm{C}_{\mathrm{C}}^{\mathrm{d}} \mathrm{b}_{\mathrm{C}}^{\mathrm{p}}$

$\diamond$ if $\gamma<30^{\circ}$ then $\angle A C_{C}^{d} B=180^{\circ}-2\left[120^{\circ}-(\alpha+\beta)\right]=60^{\circ}-2 \gamma=s\left(60^{\circ}-2 \gamma\right)$,

$\diamond$ if $\gamma>30^{\circ}$ then $\angle A C_{C}^{\mathrm{d}} \mathrm{B}=180^{\circ}-2\left[60^{\circ}+(\alpha+\beta)\right]=2 \gamma-60^{\circ}-s\left(60^{\circ}-2 \gamma\right)$.

So for the cases $\gamma<30^{\circ}$ and $\gamma>30^{\circ}$ have respectively:

$\diamond \angle A C_{C}^{\mathrm{d}} \mathrm{B}=s\left(60^{\circ}-2 \gamma\right)$ and

$\diamond \angle A C_{C}^{p} B=360^{\circ}-(\alpha+\gamma)^{+}-(\beta+\gamma)^{+}=120^{\circ}-\gamma=90^{\circ}+\frac{1}{2}\left(60^{\circ}-2 \gamma\right)=90^{\circ}+\frac{1}{2} s \angle A C_{C}^{d} B$.

Therefore from (1) and (2), by the Incenter Lemma $\left(\gamma<30^{\circ}, s=1\right)$ or the Excenter Lemma $\left(\gamma>30^{\circ}, s=-1\right), C_{C}^{p}$ is the incenter or the excenter of $\triangle A C_{C}^{d} B$ respectively. Thus $\angle C_{C}^{p} A b_{C}^{p}=\angle C_{C}^{p} A B$ and $\angle C_{C}^{p} B a a_{C}^{p}=\angle C_{C}^{p} B A$.

Moreover from $\triangle C_{C}^{p} A b_{C}^{p}$ and $\triangle C_{C}^{p} B a_{C}^{p}$ infer $\angle C_{C}^{p} A b_{C}^{p}=\gamma+\beta$ and $\angle C_{C}^{p} B a_{C}^{p}=\gamma+\alpha$.

Deduce for $\gamma \neq 30^{\circ}$ it holds $\angle C_{C}^{p} A b_{C}^{p}=\angle C_{C}^{p} A B=\gamma+\beta$ and $\angle C_{C}^{p} B a_{C}^{p}=\angle C_{C}^{p} B A=\gamma+\alpha$.

$\triangleright$ Assume $\gamma=30^{\circ}$ and so $\alpha+\beta=30^{\circ}$. Then the choice of angles in construction of $\triangle \mathrm{C}_{\mathrm{C}}^{\mathrm{p}} \mathrm{Ba}_{\mathrm{C}}^{\mathrm{p}}$ and $\triangle C_{C}^{p} A b_{C}^{p}$ implies $\angle C_{C}^{p} b_{C}^{p} A=\angle C_{C}^{p} a_{C}^{p} B=\alpha+\beta=30^{\circ}$. Hence $A b_{C}^{p}, B a_{C}^{p} \perp a_{C}^{p} b_{C}^{p}$ and thus $A b_{C}^{p} / / B a_{C}^{p}$. Draw from $C_{C}^{p}$ the height of the equilateral $\triangle a_{C}^{p} b_{C}^{p} C_{C}^{p}$ meeting $A B$ at $M$. Hence $C_{C}^{p} M / / A b_{C}^{p} / / B a_{C}^{p}$, and also $M C_{C}^{p}$ bisects $a_{C}^{p} b_{C}^{p}$. But so $M$ is the midpoint of $A B$. Also notice that $\triangle A C_{C}^{p} B$ is right triangle as $\angle A C_{C}^{p} B=360^{\circ}-60^{\circ}-\left(\beta+30^{\circ}\right)^{+}-\left(\alpha+30^{\circ}\right)^{+}=90^{\circ}$. Then $C_{C}^{p} M=M A=M B$. Now $C_{C}^{p} M=M A$ implies $\angle C_{C}^{p} A M=\angle M C_{C}^{p} A$. Yet $C_{C}^{p} M / / A b_{C}^{p}$ implies $\angle C_{C}^{p} A b_{C}^{p}=\angle A C_{C}^{p} M$. Thus $\angle C_{C}^{p} A b_{C}^{p}=\angle C_{C}^{p} A M$ and so $A C_{C}^{p}$ is the $\angle b_{C}^{p} A B$ bisector.

Similarly it is shown that $B C_{C}^{p}$ is the $\angle a_{C}^{p} B A$ bisector.

Also the choice of angles in the construction of $\triangle C_{C}^{p} A b_{C}^{p}$ gives

$$
\angle C_{\mathrm{C}}^{\mathrm{p}} A \mathrm{~b}_{\mathrm{C}}^{\mathrm{p}}=180^{\circ}-30^{\circ}-\left(\alpha+30^{\circ}\right)^{+}=30^{\circ}+\beta .
$$

Deduce for $\gamma=30^{\circ}$ it holds $\angle C_{C}^{p} A b_{C}^{p}=\angle C_{C}^{p} A B=30^{\circ}+\beta=\gamma+\beta$.

Similarly it is shown that $\angle \mathrm{C}_{\mathrm{C}}^{\mathrm{p}} \mathrm{Ba}_{\mathrm{C}}^{\mathrm{p}}=\angle \mathrm{C}_{\mathrm{C}}^{\mathrm{p}} \mathrm{BA}=\gamma+\alpha$.

$\circ$ Conclude for all $\gamma$ it holds

$$
\angle C_{C}^{p} A b_{C}^{p}=\angle C_{C}^{p} A B=30^{\circ}+\beta=\gamma+\beta \text { and } \angle C_{C}^{p} B a_{C}^{p}=\angle C_{C}^{p} B A=\gamma+\alpha .
$$

From $\triangle A b_{C}^{p} C$ it follows $\angle b_{C}^{p} A C=180^{\circ}-\beta-\gamma$, since from the construction choice of angles $\angle A b_{C}^{p} C=\beta$ and $\angle A C b_{C}^{p}=\gamma$, as found in the first step. But clearly $\angle b_{C}^{p} A C=\angle b_{C}^{p} A C_{C}^{p}+$ $\angle C_{C}^{p} A B+\angle C A B=2(\gamma+\beta)+\angle A$. Then $\angle A=3 \alpha$ and similarly $\angle B=3 \beta$. Therefore the angles of $\triangle A B C$ are $3 \alpha, 3 \beta$ and $3 \gamma$. Since $\angle C_{C}^{p} A b_{C}^{p}=\angle C_{C}^{p} A B=\gamma+\beta$ it follows that $A b_{C}^{p}$ and $A C_{C}^{p}$ 
are trisectors of $\angle A$ in $\triangle A B C$.

Similarly it shown that $\mathrm{Ba}_{\mathrm{C}}^{\mathrm{p}}$ and $B C_{C}^{\mathrm{p}}$ are trisectors of $\angle \mathrm{B}$ in $\triangle A B C$.

Corollary 3. a) In any $\triangle \mathrm{ABC}$, the angles formed by the side's of the exterior Morley equilateral $\triangle \mathrm{a}_{\mathrm{C}}^{\mathrm{p}} \mathrm{C}_{\mathrm{C}}^{\mathrm{p}} \mathrm{b}_{\mathrm{C}}^{\mathrm{p}}$ relative to the $\angle \mathrm{C}$ and the exterior trisectors of $\angle \mathrm{A}$ and $\angle \mathrm{B}$ are: $\angle \mathrm{a}_{\mathrm{C}}^{\mathrm{p}} \mathrm{C}_{\mathrm{C}}^{\mathrm{p}} \mathrm{B}=$ $(\gamma+\beta)^{+}, \angle b_{A}^{p} C_{C}^{p} A=(\gamma+\alpha)^{+}, \angle C_{C}^{p} a_{C}^{p} B=\angle C_{C}^{p} b_{A}^{p} A=\alpha+\beta$, while with the interior trisectors of $\angle \mathrm{C}$ are: $\angle \mathrm{C}_{\mathrm{C}}^{\mathrm{p}} \mathrm{a}_{\mathrm{C}}^{\mathrm{p}} \mathrm{C}=\beta$ and $\angle \mathrm{C}_{\mathrm{C}}^{\mathrm{p}} \mathrm{b}_{\mathrm{C}}^{\mathrm{p}} \mathrm{C}=\alpha$.

b) The heights of the equilateral $\triangle \mathrm{a}_{\mathrm{C}}^{\mathrm{p}} \mathrm{C}_{\mathrm{C}}^{\mathrm{p}} \mathrm{b}_{\mathrm{C}}^{\mathrm{p}}$ are: $\mathrm{a}_{\mathrm{C}}^{\mathrm{p}} \mathrm{a}_{\mathrm{C}}^{\mathrm{d}}, \mathrm{b}_{\mathrm{C}}^{\mathrm{p}} \mathrm{b}_{\mathrm{C}}^{\mathrm{d}}$ and $\mathrm{C}_{\mathrm{C}}^{\mathrm{p}} \mathrm{C}_{\mathrm{C}}^{\mathrm{d}}$.

\section{Implications}

\subsection{Companion Equilaterals of the inner Morley equilateral}

Theorem 4. The two sides' extensions of the inner Morley equilateral meet the corresponding inner trisectors at two points which with the two sides' common vertex form an equilateral.

Proof. As usually $\triangle A^{p} B^{p} C^{p}$ denotes the interior Morley equilateral of $\triangle A B C$. Let $S_{A}$ be the intersection of the extension of side $A^{p} C^{p}$ with the trisector $C^{p}$ and let $K_{A}$ be the intersection of the extension of side $A^{p} B^{p}$ with the trisector $B C^{p}$. Moreover let $A^{d}$ be the intersection of the trisectors $\mathrm{BC}^{\mathrm{p}}$ and $\mathrm{CB}^{\mathrm{p}}$. By Corollary $1 \mathrm{a}, \angle \mathrm{A}^{\mathrm{p}} \mathrm{B}^{\mathrm{p}} \mathrm{C}=\angle \mathrm{C}^{\mathrm{p}} \mathrm{A}^{\mathrm{p}} \mathrm{B}=\alpha^{+}$ and so $\triangle B^{p} A^{d} C^{p}$ is isosceles. Thus $A^{p} A^{d}$ is bisector of both $\angle B^{p} A^{p} C^{p}$ and $\angle B^{p} A^{d} C^{p}$.

Hence $\angle B^{p} A^{p} A^{d}=\angle C^{p} A^{p} A^{d}$ and $\angle B^{p} A^{d} A^{p}=\angle C^{p} A^{d} A^{p}$. Also $\angle S_{A} A^{d} A^{p}=\angle K_{A} A^{d} A^{p}$ as obviously $\angle S_{A} A^{d} C^{p}=$ $\angle \mathrm{K}_{\mathrm{A}} A^{\mathrm{d}} \mathrm{B}^{\mathrm{p}}$ and $\angle \mathrm{S}_{\mathrm{A}} A^{\mathrm{d}} A^{\mathrm{p}}=\angle \mathrm{S}_{\mathrm{A}} A^{\mathrm{d}} \mathrm{C}^{\mathrm{p}}+\angle \mathrm{C}^{\mathrm{p}} \mathrm{A}^{\mathrm{d}} A^{\mathrm{p}}$ while $\angle \mathrm{K}_{\mathrm{A}} \mathrm{A}^{\mathrm{d}} \mathrm{A}^{\mathrm{p}}=\angle \mathrm{K}_{\mathrm{A}} \mathrm{A}^{\mathrm{d}} \mathrm{A}^{\mathrm{p}}+\angle \mathrm{B}^{\mathrm{p}} \mathrm{A}^{\mathrm{d}} \mathrm{A}^{\mathrm{p}}$.

Therefore $\triangle A^{p} S_{A} A^{d}$ and $\triangle A^{p} K_{A} A^{d}$ are equal because in

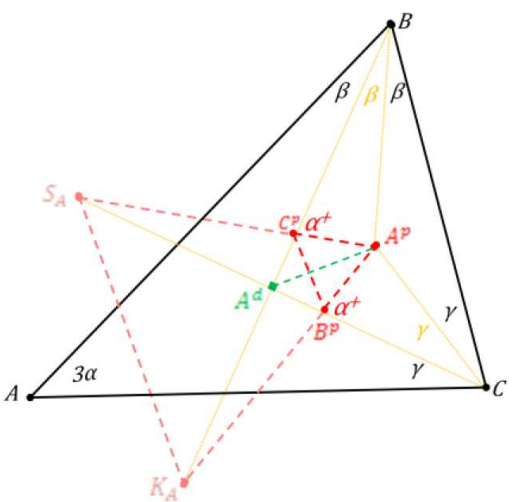

Fig.12 addition they have side $A^{p} A^{d}$ in common. Consequently $\triangle S_{A} A^{p} K_{A}$ is equilateral.

The previous equilateral is named companion equilateral relative to vertex $A^{p}$ and it will be denoted by $\triangle S_{A} A^{p} K_{A}$. Obviously there are two more companion equilaterals relative to vertices $\mathrm{B}^{p}$ and $\mathrm{C}^{p}$, denoted by $\triangle \mathrm{S}_{\mathrm{B}} \mathrm{B}^{p} \mathrm{~K}_{\mathrm{B}}$ and $\triangle \mathrm{S}_{\mathrm{C}} \mathrm{C}^{p} \mathrm{~K}_{\mathrm{C}}$, respectively.

Corollary 4. For the companion equilateral relative to vertex $A^{p}, \triangle S_{A} A^{p} K_{A}$, it holds

$$
\angle \mathrm{BAS}_{\mathrm{A}}=\angle \mathrm{CAK}_{\mathrm{A}}=|\beta+\gamma-\alpha| \text {. }
$$


In fact, the points $S_{A}$ and $\mathrm{K}_{\mathrm{A}}$, for $\alpha<30^{\circ}$ are outside $\triangle \mathrm{ABC}$, for $\alpha=30^{\circ}$ are on $\mathrm{AB}$ and $\mathrm{AC}$, respectively, and for $\alpha>30^{\circ}$ are inside $\triangle \mathrm{ABC}$.

Proof. Corollary 1a asserts $\angle C^{p} A^{p} B=\gamma^{+}$and $\angle B^{p} A^{p} C=\beta^{+}$. Thus from $\triangle K_{A} A^{p} B$ and $\triangle K_{A} A^{p} B$ it is calculated $\angle C^{p} S_{A} B^{p}=\alpha$ and $\angle C^{p} K_{A} B^{p}=\alpha$, respectively. Then the points $B^{p}, C^{p}, S_{A}$ and $K_{A}$ are cyclic as $B^{p} C^{p}$ is seen from $S_{A}$ and $K_{A}$ with the same angle. Thus $\angle A S_{A} K_{A}=\angle A B^{p} K_{A}$ and $\angle A K_{A} S_{A}=\angle A C^{p} S_{A}$. From $\triangle B C^{p} A$ and $\triangle C B^{p} A$ infer that $\angle A C^{p} K_{A}=\beta+\gamma$ and $\angle A B^{p} S_{A}=\alpha+\gamma$, respectively. Hence $\angle A S_{A} K_{A}=\beta+\gamma$ and $\angle A K_{A} S_{A}=\alpha+\gamma$.

Next notice that $\angle S_{A} A C^{p}=180^{\circ}-\angle A S_{A} C^{p}-$ $\angle S_{A} C^{p} A=180^{\circ}-\left(60^{\circ}+\beta+\gamma\right)-(\alpha+\gamma)=\beta+\gamma$ and similarly $\angle \mathrm{K}_{\mathrm{A}} A C=\beta+\gamma$.

However

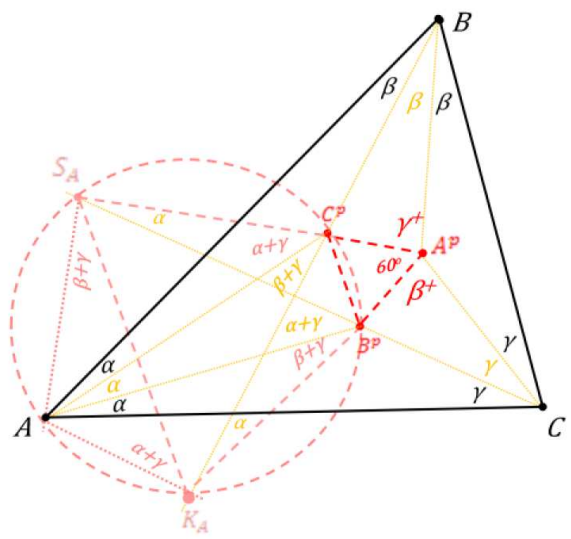

Fig.13

$$
\begin{aligned}
& \angle \mathrm{S}_{\mathrm{A}} A C^{\mathrm{p}}=\angle \mathrm{S}_{\mathrm{A}} \mathrm{AB} \pm \angle \mathrm{BAC} \mathrm{C}^{\mathrm{p}} \text { and } \\
& \angle \mathrm{K}_{\mathrm{A}} A \mathrm{~A}=\angle \mathrm{K}_{\mathrm{A}} A C \pm \angle C A B^{p},
\end{aligned}
$$

where \pm may be either + or - depending on the location of $S_{A}$ and $K_{A}$ with respect to $A B$ and $A C$ respectively. Therefore $\angle S_{A} A B=\angle K_{A} A C=|\beta+\gamma-\alpha|$.

Because $\alpha+\beta+\gamma=60^{\circ}$ for $\alpha<30^{\circ}$ the points $S_{A}$ and $K_{A}$ are outside $\triangle A B C$, for $\alpha=30^{\circ}$ the points $S_{A}$ and $K_{A}$ are on $A B$ and $A C$, respectively and for $\alpha>30^{\circ}$ the points $S_{A}$ and $K_{A}$ are inside $\triangle A B C$.

\subsection{Relation of the Morley equilaterals}

Theorem 5. In any triangle the sides of Morley equilaterals are either collinear or parallel.

Proof. Corollary 2a claims $\angle B C_{C}^{p} A_{A}^{p}=\alpha$. Also Corollary 3a confirms $\angle B C_{C}^{p} a_{C}^{p}=(\beta+\gamma)^{+}$. Hence $\angle B_{B}^{p} C_{C}^{p} a_{C}^{p}=\angle B_{B}^{p} C_{C}^{p} A_{A}^{p}+\angle A_{A}^{p} C_{C}^{p} B+\angle B C_{C}^{p} a_{C}^{p}=$ $=60^{\circ}+\alpha+(\beta+\gamma)^{+}=180^{\circ}$.

Thus $a_{C}^{p} C_{C}^{p}$ is extension of $B_{B}^{p} C_{C}^{p}$. Similarly it is shown that $b_{C}^{p} C_{C}^{p}$ is extension of $A_{A}^{p} C_{C}^{p}$.

As $\angle b_{C}^{p} a_{C}^{p} C_{C}^{p}=\angle A_{A}^{p} B_{B}^{p} C_{C}^{p}=60^{\circ}$, it follows $a_{C}^{p} b_{C}^{p} / / A_{A}^{p} B_{B}^{p}$.

Since $\angle b_{C}^{p} a_{C}^{p} C=\angle b_{C}^{p} a_{C}^{p} C_{C}^{p}+\angle C_{C}^{p} a_{C}^{p} C=60^{\circ}+\beta$

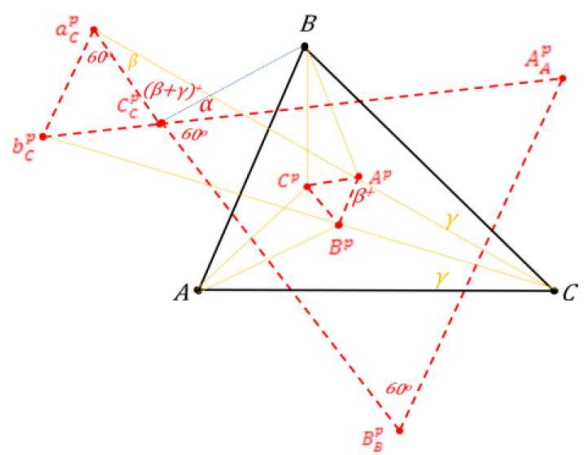

Fig.14 
and $\angle B^{p} A^{p} C=\beta^{+}$then

$$
a_{C}^{p} b_{C}^{p} / / A^{p} B^{p}
$$

The previous result is mentioned as a fact in [8] and it might be in print elsewhere. At any event, it inspires the next theorem.

\subsection{Interrelationship between central and exterior Morley equilaterals}

Theorem 6. In any triangle, the exterior trisectors of its angles, proximal to the three sides respectively, meet at the vertices of an equilateral, if and only if, the interior trisectors of an angle and the exterior trisectors of the other two angles, proximal the three sides respectively, meet at the vertices of an equilateral.

Proof. Let $\triangle A B C$ be given with $\angle A=3 \alpha, \angle B=3 \beta$ and $\angle \mathrm{C}=3 \gamma$, where $\alpha+\beta+\gamma=60^{\circ}$.

$(\Longrightarrow)$ Assume that $\triangle A_{A}^{p} B_{B}^{p} C_{C}^{p}$ is the central Morley equilateral formed by the intersections of the exterior trisectors, proximal to the sides of $\triangle A B C$. See Fig.15a.

Extend $A_{A}^{p} C_{C}^{p}$ and $B_{B}^{p} C_{C}^{p}$ to meet the extensions of $A_{A}^{p} B$ and $B_{B}^{p} A$ at $A^{\prime \prime}$ and $B^{\prime \prime}$ respectively. Then $A B^{\prime \prime}$ and $B A^{\prime \prime}$ are trisectors of the exterior $\angle A$ and $\angle B$ respectively, as extensions of the corresponding trisectors. Thus it suffices to show that $\triangle A^{\prime \prime} C_{C}^{p} B^{\prime \prime}$ is equilateral and also that

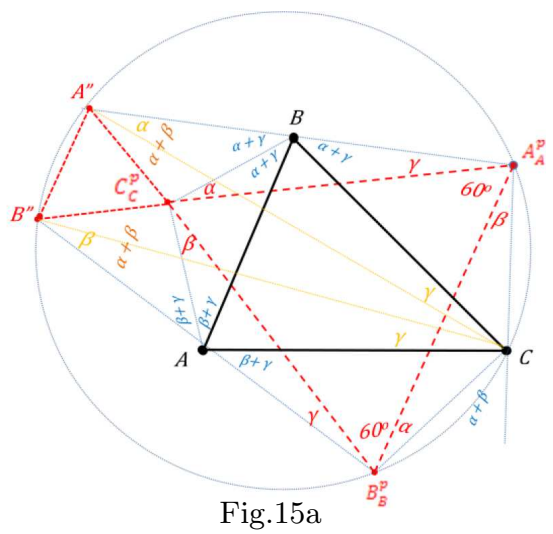
$\mathrm{CA}^{\prime \prime}$ and $\mathrm{CB}^{\prime \prime}$ are trisectors of the interior $\angle \mathrm{C}$.

First show that $\triangle A^{\prime \prime} C_{C}^{p} B^{\prime \prime}$ is equilateral.

Notice that $\triangle A^{\prime \prime} A_{A}^{p} B_{B}^{p}=\triangle B^{\prime \prime} A_{A}^{p} B_{B}^{p}$ because they have $A_{A}^{p} B_{B}^{p}$ common, $\angle A^{\prime \prime} B_{B}^{p} A_{A}^{p}=\angle B^{\prime \prime} A_{A}^{p} B_{B}^{p}=60^{\circ}$ and $\angle A^{\prime \prime} A_{A}^{p} B_{B}^{p}=\angle B^{\prime \prime} B_{B}^{p} A_{A}^{p}=60^{\circ}+\gamma$ by Corollary 2a. Thus $A^{\prime \prime} B_{B}^{p}=B^{\prime \prime} A_{A}^{p}$. But so $C_{C}^{p} A^{\prime \prime}=C_{C}^{p} B^{\prime \prime}$, as $C_{C}^{p} A_{A}^{p}=C_{C}^{p} B_{B}^{p}$ since $\triangle A_{A}^{p} B_{B}^{p} C_{C}^{p}$ is equilateral. Also $\angle A^{\prime \prime} C_{C}^{p} B^{\prime \prime}=\angle A_{A}^{p} C_{C}^{p} B_{B}^{p}=60^{\circ}$ and hence $\triangle A^{\prime \prime} B_{B}^{p} C_{C}^{p}$ is equilateral.

Next show that $C A^{\prime \prime}$ and $C B^{\prime \prime}$ are trisectors of the interior $\angle C$.

From $\triangle A_{A}^{p} A^{\prime \prime} B_{B}^{p}$ and $\triangle A_{A}^{p} B^{\prime \prime} B_{B}^{p}$ it is easily calculated that $\angle A_{A}^{p} A^{\prime \prime} B_{B}^{p}=\alpha+\beta$ and $\angle A B^{\prime \prime} B_{B}^{p}=$ $\alpha+\beta$. Hence $A_{A}^{p}, B_{B}^{p}, A^{\prime \prime}, B^{\prime \prime}$ are cyclic. But $\angle B_{B}^{p} A_{A}^{p} C=\beta$ and $\angle A_{A}^{p} B_{B}^{p} C=\alpha$, by Corollary 2a. So from $\triangle A_{A}^{p} C B_{B}^{p}, \angle A_{A}^{p} C B_{B}^{p}=180^{\circ}-(\alpha+\beta)$. But so $C$ is also on this circle. Thus $\angle C A^{\prime \prime} A_{A}^{p}=\angle A_{A}^{p} B_{B}^{p} C=\alpha$ and $\angle C B^{\prime \prime} B_{B}^{p}=\angle B_{B}^{p} A_{A}^{p} C=\beta$. Finally note that in $\triangle B A^{\prime \prime} C$ and $\triangle C B^{\prime \prime} A$ it holds respectively $\angle A^{\prime \prime} C B=(\alpha+\gamma)-\alpha=\gamma$ and $\angle B^{\prime \prime} C A=(\beta+\gamma)-\beta=\gamma$.

Conclude $\mathrm{CA}^{\prime \prime}$ and $\mathrm{CB}^{\prime \prime}$ are trisectors of the interior $\angle \mathrm{C}$, as $\angle \mathrm{C}=3 \gamma$. 
$(\Longleftarrow)$ Assume that the exterior triangle $\Delta \mathrm{a}_{\mathrm{C}}^{\mathrm{p}} \mathrm{C}_{\mathrm{C}}^{\mathrm{p}} \mathrm{b}_{\mathrm{C}}^{\mathrm{p}}$ is equilateral formed by the intersections of the trisectors of the interior $\angle C$ and the exterior $\angle A$ and $\angle B$. See Fig.15b.

Extend $a_{C}^{p} C_{C}^{p}$ and $b_{C}^{p} c_{C}^{p}$ to meet the extensions of $a_{C}^{p} B$ and $b_{C}^{p} A$ at $A^{\prime \prime}$ and $B^{\prime \prime}$ respectively. Then $A B^{\prime \prime}$ and $B A^{\prime \prime}$ are trisectors of the exterior $\angle A$ and $\angle B$ respectively, as extensions of the corresponding trisectors.

Thus it suffices to show that $\triangle A^{\prime \prime} C_{C}^{p} B^{\prime \prime}$ is equilateral

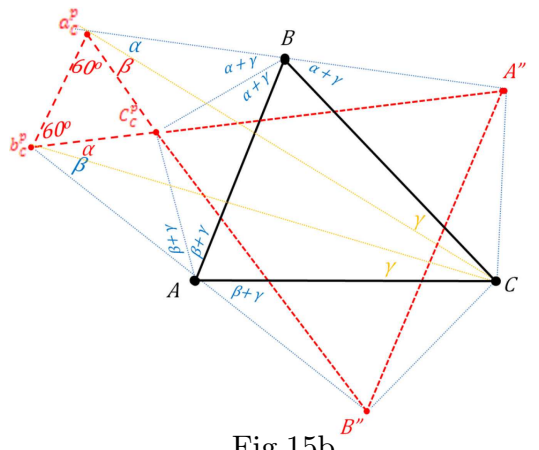
and that $\mathrm{CA}^{\prime \prime}$ and $\mathrm{CB}^{\prime \prime}$ are trisectors of the interior $\angle \mathrm{C}$.

Note $\triangle A^{\prime \prime} a_{C}^{p} b_{C}^{p}=\triangle B^{\prime \prime} a_{C}^{p} b_{C}^{p}$, because they have $a_{C}^{p} b_{C}^{p}$ common, $\angle A^{\prime \prime} B_{B}^{p} A_{A}^{p}=\angle B^{\prime \prime} A_{A}^{p} B_{B}^{p}=60^{\circ}$ and $\angle A^{\prime \prime} A_{A}^{p} B_{B}^{p}=\angle B^{\prime \prime} B_{B}^{p} A_{A}^{p}=60^{\circ}+\alpha+\beta$ by Corollary 3a. Thus $A^{\prime \prime} b_{C}^{p}=B^{\prime \prime} a_{C}^{p}$. Since $\triangle a_{C}^{p} C_{C}^{p} b_{C}^{p}$ is equilateral then $C_{C}^{p} A^{\prime \prime}=C_{C}^{p} B^{\prime \prime}$ and so $\triangle A^{\prime \prime} C_{C}^{p} B^{\prime \prime}$ is equilateral.

Also from $\triangle a_{C}^{p} B C$ and $\triangle b_{C}^{p} C A$ it is calculated that $\angle a_{C}^{p} C B=\angle b_{C}^{p} C A=\gamma$ and so $C a_{C}^{p}$ and $\mathrm{Cb}_{\mathrm{C}}^{\mathrm{p}}$ are trisectors of $\angle \mathrm{C}$.

\section{The non Equilateral Morley Triangles}

For a given $\triangle A B C$ there are in general 64 Morley triangles, as the trisectors of its three angles meet at many points. Among them are the inner, the central and the exterior Morley equilaterals.

A number of authors (see for example [4] or [5]) have wondered: Are there more Morley equilaterals for $\triangle \mathrm{ABC}$ ?

This part examines all the remaining Morley triangles of $\triangle A B C$ systematically and shows that none of them is equilateral. used.

In the sequel the following easily proved lemma is

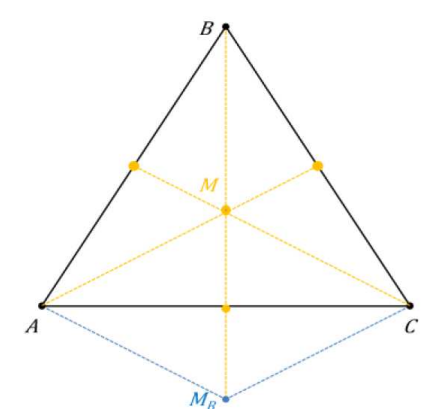

Fig.16

The Equilateral Center Lemma. The incenter of an equilateral is the unique interior point from which its sides are seen with $120^{\circ}$. Similarly the excenter relative to an angle is the unique exterior point from which the side opposite to the angle is seen with $120^{\circ}$ while the other two sides are seen with $60^{\circ}$.

\subsection{Morley triangles by trisectors of interior angles}

This section treats the non equilateral Morley triangles formed by the trisectors of the interior angles of $\triangle A B C$. The proximal to the sides trisectors meet at $A^{p}, B^{p}$ and $C^{p}$ and $\triangle A^{p} B^{p} C^{p}$ 
denotes the inner Morley equilateral.

\subsubsection{The Interior Morley triangle of distal vertices}

The interior Morley triangle of distal vertices is denoted by $\triangle A^{d} B^{d} C^{d}$ where $A^{d}, B^{d}$ and $C^{d}$ are the meeting points of the distal trisectors with respect to the sides $B C, C A$ and $A B$, respectively, as shown in Fig.17. If $\triangle A B C$ is equilateral then $\triangle A^{d} B^{d} C^{d}$ is equilateral as well. Thus in the following we assume that $\triangle A B C$ is not equilateral.

From Corollary $1 b$, we have that $A^{p} A^{d}$, $B^{p} B^{d}$ and $C^{p} C^{d}$ are the heights of the inner Morley equilateral $\triangle A^{p} B^{p} C^{p}$.

Let $M$ be the center of $\triangle A^{p} B^{p} C^{p}$. Thus $\angle A^{p} M B^{d}=\angle B^{d} M C^{p}=\angle C^{p} M A^{d}=\angle A^{d} M B^{p}$

$$
=\angle B^{p} M C^{d}=\angle C^{d} M A^{p}=60^{\circ}
$$

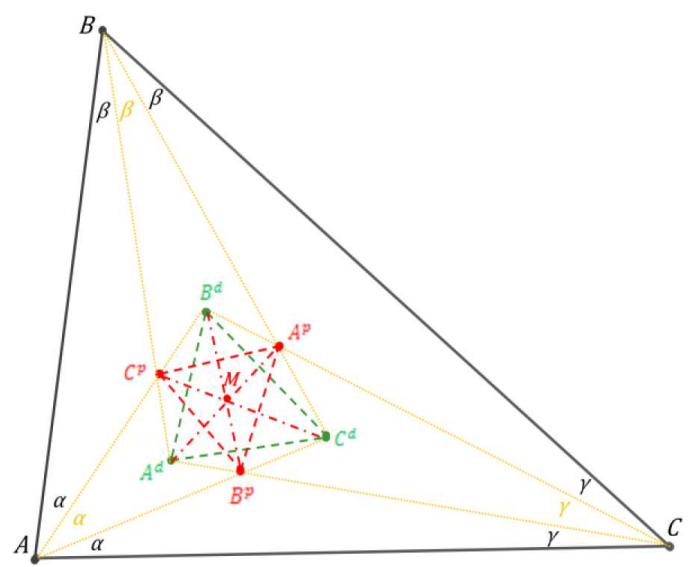

Fig. 17

So $\angle A^{d} M B^{d}=\angle B^{d} M C^{d}=\angle C^{d} M A^{d}=120^{\circ}$.

Hence the sides of $\triangle A^{d} B^{d} C^{d}$ are seen from $M$ with $120^{\circ}$.

Assume towards a contradiction that $\triangle A^{d} B^{d} C^{d}$ is equilateral. Then, by the Equilateral Center Lemma, $A^{p} A^{d}$ is a height of $\triangle A^{d} B^{d} C^{d}$. Thus $A^{p} A^{d}$ bisects $B^{d} C^{d}$. Hence $A^{p} A^{d}$ bisects $\angle B^{d} A^{p} C^{d}$ and so $\angle B A^{p} C$. Since $A^{p}$ is the incenter of $\triangle B A^{d} C, A^{p} A^{d}$ bisects also $\angle B A{ }^{d} C$. But so the exterior angles of $\triangle A^{d} A^{p} B$ and $\triangle A^{d} A^{p} C$ at vertex $A^{p}$ are $\frac{1}{2} \angle B A^{p} C=\frac{1}{2} \angle B A^{d} C+\beta$ and $\frac{1}{2} \angle B A^{p} C=\frac{1}{2} \angle B A^{d} C+\gamma$. Hence $\beta=\gamma$. Similarly it is shown that $\alpha=\beta$. Thus $\triangle A B C$ is equilateral contrary to the assumption.

Conclude that $\triangle A^{d} B^{d} C^{d}$ cannot be equilateral (if $\triangle A B C$ is not equilateral).

\subsubsection{Interior Morley triangles with one proximal and two mix vertices}

There are three interior Morley triangles with one proximal and two mix vertices denoted by $\triangle A^{p} B^{*} C^{*}, \triangle B^{p} C^{*} A^{*}$ and $\triangle C^{p} A^{*} B^{*}$. We will study only $\triangle A^{p} B^{*} C^{*}$ as the other two are similar. 
Since $A^{p}$ is the intersection of the proximal trisectors, $B^{*}$ must be the intersection of the remaining trisector $\mathrm{CB}^{p}$ (proximal to $C A$ ) with $A C^{p}$ as distal. Then $C^{*}$ is the intersection of the left trisectors $B C^{p}$ (distal to $A B$ ) and $A B^{p}$ (proximal). So $B^{*}$ is on $A C^{p}$ and $C^{*}$ is on $A B^{p}$. See Fig.18. Corollary 1a asserts $\angle A C^{p} B^{p}=\beta^{+}$and $\angle A B^{p} C^{p}=\gamma^{+}$. Hence $\angle A C^{p} A^{p}=60^{\circ}+\beta^{+}<180^{\circ}$ and $\angle A B^{p} A^{p}=60^{\circ}+\gamma^{+}<180^{\circ}$. Therefore the quadrangle $A B^{p} A^{p} C^{p}$ is convex and so $\angle B^{*} A^{p} C^{*}$ is inside $\angle B^{p} A^{p} C^{p}$.

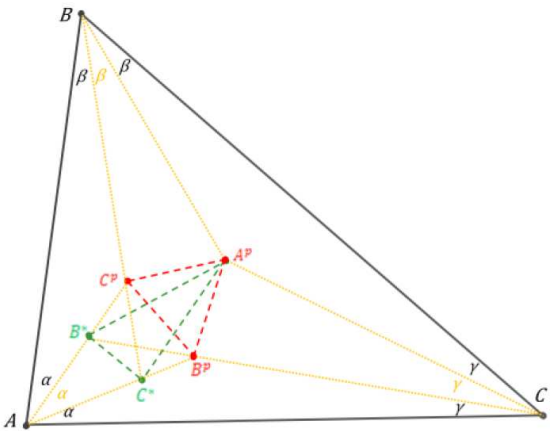

Fig.18

Conclude that $\angle \mathrm{B}^{*} \mathrm{~A}^{p} \mathrm{C}^{*}<60^{\circ}$ and thus $\triangle \mathrm{A}^{\mathrm{p}} \mathrm{B}^{*} \mathrm{C}^{*}$ cannot be equilateral.

\subsubsection{Interior Morley triangles with one distal and two mix vertices}

There are three interior Morley triangles with one distal and two mix vertices which are denoted by $\triangle A^{d} B^{*} C^{*}, \triangle B^{d} C^{*} A^{*}$ and $\triangle C^{d} A^{*} B^{*}$. We will study only $\triangle A^{d} B^{*} C^{*}$ as the other two are similar. Since $A^{d}$ is the intersection of the distal trisectors, $\mathrm{B}^{*}$ must be the intersection of the remaining trisectors $C A^{p}$ (distal to $C A$ ) and $A B^{p}$, as proximal. So $C^{*}$ is the intersection of the left trisectors $A C^{p}$ and $B A^{p}$ (mix to $A B$ ).

First notice that if $\beta=\gamma$ then $\triangle A^{d} B^{*} C^{*}$ is isosceles, because Corollary 1a with $\beta=\gamma$ yields $\triangle B^{p} A^{p} B^{*}=\triangle C^{p} A^{p} C^{*}$ and so $A^{p} B^{*}=$ $A^{p} C^{*}$ which implies $\triangle A^{d} A^{p} B^{*}=\triangle A^{d} A^{p} C^{*}$.

Thus if $\alpha=\beta=\gamma$ then $\triangle A^{d} B^{*} C^{*}$ is equi-

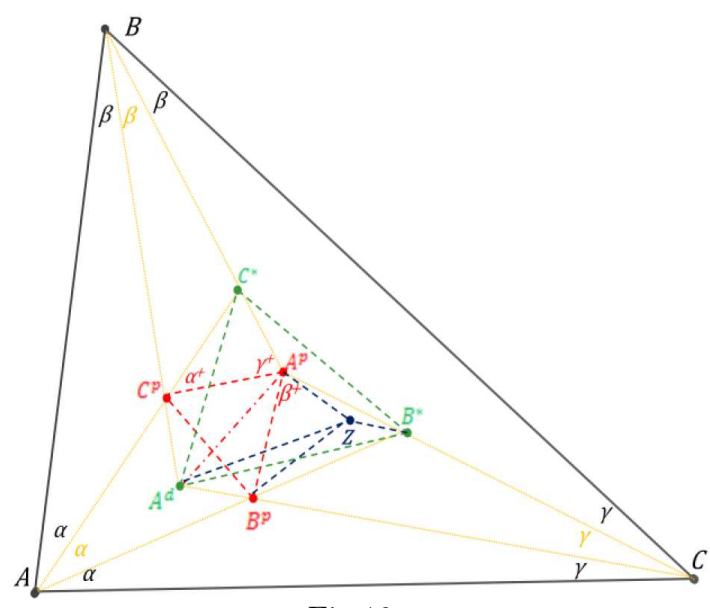

Fig.19 lateral.

Assume $\triangle A B C$ is not equilateral. Then it has two sides not equal and thus in the following we may assume $\gamma<\beta$. Fig.19.

Suppose, towards a contradiction, that $A^{d} B^{*}=A^{d} C^{*}$. Let $Z$ be the symmetric point of $C^{*}$ with respect to $A^{p} A^{d}$. We will fist show that $Z$ is inside $\triangle A^{d} A^{p} B^{*}$.

From Corollary $1 b, A^{p} A^{d}$ is height of the equilateral $\triangle A^{p} B^{p} C^{p}$ and so $B^{p}$ and $C^{P}$ are symmetric with respect to $A^{p} A^{d}$. Consequently $\angle B^{p} A^{p} Z=\angle C^{p} A^{p} C^{*}$ and since $\angle C^{p} A^{p} C^{*}=\gamma^{+}$infer $\angle B^{p} A^{p} Z=\gamma^{+}$. Since $\angle B^{p} A^{p} B^{*}=\beta^{+}$and by assumption $\gamma<\beta$, deduce $\angle B^{p} A^{p} Z<\angle B^{p} A^{p} B^{*}$. Moreover $\angle A^{p} B^{p} Z=\angle A^{p} C^{p} C^{*}=\alpha+(\alpha+\beta)=\alpha+\gamma$ while $\angle A^{p} B^{p} B^{*}=\alpha+(\alpha+\gamma)=\alpha+\beta$. So $\angle A^{p} B^{p} Z<\angle A^{p} B^{p} B^{*}$. 
Since $Z$ is inside $\triangle A^{d} A^{p} B^{*}$ then $\angle A^{d} Z B^{*}>\angle A^{d} A^{p} B^{*}=\angle A^{d} A^{p} B^{p}+\angle B^{p} A^{p} B^{*}=30^{\circ}+$ $\beta^{+}=90^{\circ}+\beta$. However, the assumption $A^{d} B^{*}=A^{d} C^{*}$ implies $A^{d} Z=A^{d} B^{*}$ and so $\angle A^{d} Z B^{*}=$ $\angle A^{d} B^{*} Z$. Thus $\angle A^{d} Z B^{*}+\angle A^{d} B^{*} Z>2\left(90^{\circ}+\beta\right)>180^{\circ}$. Hence two angles of $\triangle A^{d} Z B^{*}$ have sum greater than $180^{\circ}$, which is a contradiction.

Conclude that $\triangle A^{d} B^{*} C^{*}$ cannot be equilateral (if $\triangle A B C$ is not equilateral).

\subsection{Morley triangles by trisectors of exterior angles}

This section treats the non equilateral Morley triangles formed by the trisectors of the exterior angles. So throughout this section trisectors mean trisectors of the exterior angles of $\triangle A B C$.

The proximal trisectors meet at the points $A_{A}^{p}, B_{B}^{p}$ and $C_{C}^{p}$ and so $\triangle A_{A}^{p} B_{B}^{p} C_{C}^{p}$ denotes the central Morley equilateral. Notice that the trisectors $B C_{C}^{p}$ and $C B_{B}^{p}$ are parallel iff

$$
\angle \mathrm{B}_{\mathrm{B}}^{\mathrm{p}} \mathrm{CB}+\angle \mathrm{C}_{\mathrm{C}}^{\mathrm{p}} \mathrm{BC}=180^{\circ} \Leftrightarrow 2(\alpha+\beta)+2(\alpha+\gamma)=180^{\circ} \text {. }
$$

So for $\alpha=30^{\circ} B C_{C}^{p} / / C_{B}^{p}$. In this case the distal trisectors with respect to $B C$ do not intersect and hence the distal to $B C$ vertex $A_{A}^{d}$ is not determined. Also if $30^{\circ}>\alpha$ then $A_{A}^{d}$ and $A_{A}^{p}$ are on the same side of $B C$ while $\angle B A_{A}^{d} C=60^{\circ}-2 \alpha$. If $30^{\circ}<\alpha$ then $A_{A}^{d}$ and $A_{A}^{p}$ are on different sides of $B C$ while $\angle B A_{A}^{d} C=2 \alpha-60^{\circ}$. See Fig. 20 .

\subsubsection{The Central Morley triangle of distal vertices}

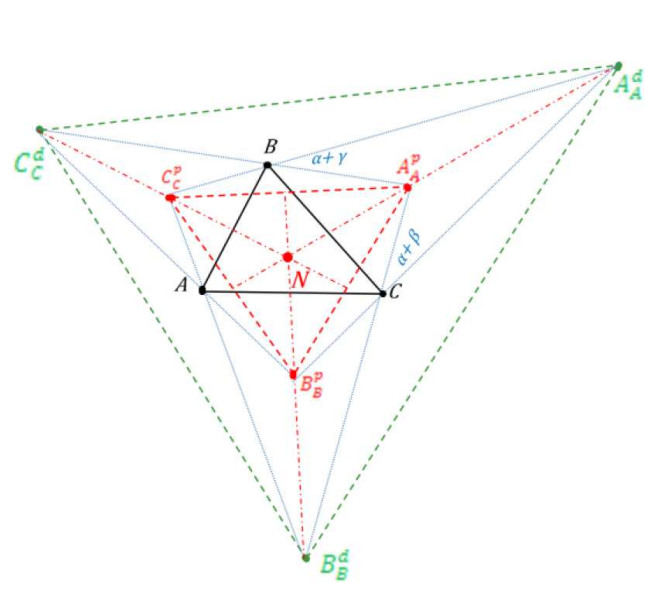

Fig.20a $\left(\gamma<30^{\circ}\right)$

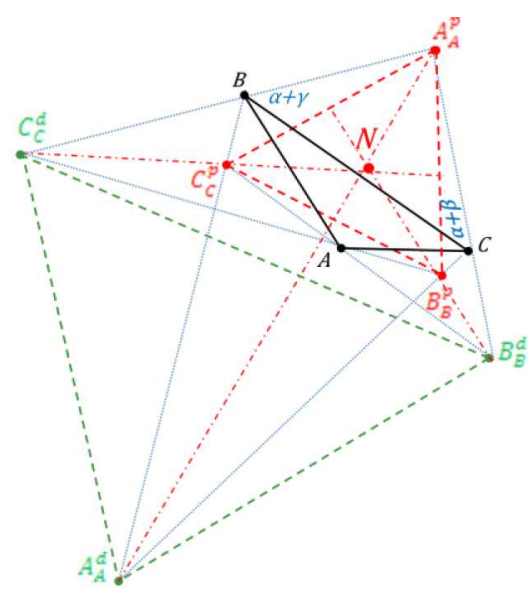

Fig.20b $\left(\gamma>30^{\circ}\right)$

The Morley triangle of distal vertices is denoted by $\triangle A_{A}^{d} B_{B}^{d} C_{C}^{d}$, where $A_{A}^{d}, B_{B}^{d}$ and $C_{C}^{d}$ are the meeting points of the distal trisectors of the exterior angles with respect to the sides $B C, C A$ and $A B$, respectively. In Fig.20a and Fig.20b the different locations of $\triangle A_{A}^{d} B_{B}^{d} C_{C}^{d}$ with respect to $\triangle A_{A}^{p} B_{B}^{p} C_{C}^{p}$ are illustrated. Note that $A_{A}^{p} A_{A}^{d}, B_{B}^{p} B_{B}^{d}$ and $C_{C}^{p} C_{C}^{d}$, by Corollary $1 b$, are the heights of the central Morley equilateral $\triangle A_{A}^{p} B_{B}^{p} C_{C}^{p}$ and let $N$ be their intersection.

Notice that if $\triangle A B C$ is equilateral then $\triangle A_{A}^{d} B_{B}^{d} C_{C}^{d}$ is equilateral as well. Thus in the following 
we assume that $\triangle A B C$ is not equilateral. Also suppose towards a contradiction that $\triangle A_{A}^{d} B_{B}^{d} C_{C}^{d}$ is equilateral.

$\triangleright$ If $\triangle A B C$ is an acute triangle the equilateral $\triangle A_{A}^{p} B_{B}^{p} C_{C}^{p}$ is inside $\triangle A_{A}^{d} B_{B}^{d} C_{C}^{d}$. Hence

$$
\angle A_{A}^{d} N B_{B}^{d}=\angle B^{d} N C_{C}^{d}=\angle C_{C}^{d} N A_{A}^{d}=120^{\circ} .
$$
Hence

$\triangleright$ If $\triangle A B C$ is an obtuse triangle (assume $\alpha>30^{\circ}$ ) $A_{A}^{d}$ and $A_{A}^{p}$ are on different sides of $B C$.

$$
\angle A_{A}^{d} N B_{B}^{d}=\angle A_{A}^{d} N C_{C}^{d}=60^{\circ} \text { and } \angle B_{B}^{d} N C_{C}^{d}=120^{\circ} .
$$

Thus, by the Equilateral Center Lemma, $N$ is the incenter (acute) or the excenter (obtuse) of the assumed equilateral $\triangle A_{A}^{d} B_{B}^{d} C_{C}^{d}$. Hence $A_{A}^{p} A_{A}^{d}$ is a height of $\triangle A_{A}^{d} B_{B}^{d} C_{C}^{d}$ and so $A_{A}^{p} A_{A}^{d}$ bisects $B_{B}^{d} C_{C}^{d}$ and $\angle B_{B}^{d} A_{A}^{p} C_{C}^{d}$.

$\triangleright$ In the case of the acute triangle, notice in $\triangle B A_{A}^{d} C$ that $A_{A}^{p}$ is the incenter while the bisector $A_{A}^{p} A_{A}^{d}$ bisects $\angle B A_{A}^{p} C$. As a result $\angle A_{A}^{d} B C=\angle A_{A}^{d} C B \Leftrightarrow 2(\alpha+\gamma)=2(\alpha+\beta) \Leftrightarrow \gamma=\beta$.

$\triangleright$ In the case of the obtuse triangle, note that since $A_{A}^{p} A_{A}^{d}$ bisects $B_{B}^{d} C_{C}^{d}$ it bisects $\angle B A_{A}^{p} C$. Also it bisects $\angle B A_{A}^{d} C$ as a height of $\triangle A_{A}^{p} B_{B}^{p} C_{C}^{p}$. Then $\triangle A_{A}^{p} B A_{A}^{d}=\triangle A_{A}^{p} C A_{A}^{d}$ and so $A_{A}^{p} B=$ $A_{A}^{p}$.

Therefore $\alpha+\gamma=\alpha+\beta$ and so $\gamma=\beta$. Similarly we show that $\alpha=\beta$.

Deduce that $\triangle A B C$ is equilateral contrary to the assumption that it is not.

Conclude that $\triangle A_{A}^{d} B_{B}^{d} C_{C}^{d}$ cannot be equilateral (if $\triangle A B C$ is not equilateral).

\subsubsection{The Central Morley triangles with one proximal and two mix vertices}

There are three Morley triangles of $\triangle A B C$ formed by exterior trisectors with one proximal and two mix vertices denoted by $\triangle A_{A}^{p} B_{B}^{*} C_{C}^{*}, \triangle B_{B}^{p} C_{C}^{*} A_{A}^{*}$ and $\triangle C_{C}^{p} A_{A}^{*} B_{B}^{*}$. We will study only $\triangle A_{A}^{p} B_{B}^{*} C_{C}^{*}$ as the other two are similar.

As vertex $A_{A}^{p}$ is the intersection of the proximal to $\mathrm{BC}$ trisectors, vertex $\mathrm{B}_{\mathrm{B}}^{*}$ is the intersection of the remaining trisector $\mathrm{CB}_{\mathrm{B}}^{\mathrm{P}}$ (proximal to $\mathrm{CA}$ ) with $A C_{\mathrm{C}}^{\mathrm{P}}$, as distal. Then vertex $C_{C}^{*}$ is the intersection of the left trisectors $A B_{B}^{P}$ (distal to $A B$ ) and $B C_{C}^{P}$ (proximal). Thus $B_{B}^{*}$ is on $C_{B}^{P}$ while $C_{C}^{*}$ is on $B C_{C}^{P}$. Using the angle values between the sides of $\triangle A_{A}^{p} B_{B}^{p} C_{C}^{p}$ and the trisectors of $\triangle A B C$ given by Corollary $2 \mathrm{a}$ it is easily deduced that all the angles of the quadrangles $B_{B}^{*} B_{B}^{p} A_{A}^{p} C_{C}^{p}$ and

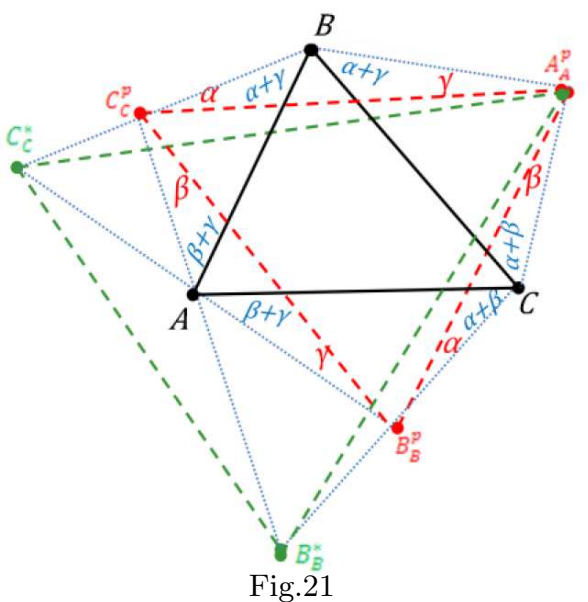


$C_{C}^{*} C_{C}^{p} A_{A}^{p} B_{B}^{p}$ are less than $180^{\circ}$ and so they are convex.

For instance

$$
\angle C_{C}^{*} C_{C}^{p} A_{A}^{p}=180^{\circ}-\angle B C_{C}^{p} A_{A}^{p}=180^{\circ}-\alpha
$$

while

$$
\angle A B_{B}^{p} A_{A}^{p}=60^{\circ}+\alpha
$$

Since $B_{B}^{*} B_{B}^{p} A_{A}^{p} C_{C}^{p}$ is convex infer $A_{A}^{p} C_{C}^{*}$ is inside $\angle B_{B}^{p} A_{A}^{p} C_{C}^{p}$. Also since $C_{C}^{*} C_{C}^{p} A_{A}^{p} B_{B}^{p}$ is convex infer $A_{A}^{p} B_{B}^{*}$ is inside $\angle B_{B}^{p} A_{A}^{p} C_{C}^{p}$.

Conclude that $\angle B_{C}^{*} A_{A}^{p} C_{C}^{*}<L B_{B}^{p} A_{A}^{p} C_{C}^{p}=60^{\circ}$ and so $\triangle A_{A}^{p} B_{B}^{*} C_{C}^{*}$ cannot be equilateral.

\subsubsection{The Central Morley triangles with one distal and two mix vertices}

There are three Morley triangles formed by exterior trisectors with one distal and two mix vertices which are denoted by $\triangle A_{A}^{d} B_{B}^{*} C_{C}^{*}, \triangle B_{B}^{d} C_{C}^{*} A_{A}^{*}$ and $\triangle C_{C}^{d} A_{A}^{*} B_{B}^{*}$. We will study only $\triangle A_{A}^{d} B_{B}^{*} C_{C}^{*}$ as the other two are similar.

Since $A_{A}^{d}$ is the intersection of the distal to $B C$ trisectors $B C_{C}^{p}$ and $C B_{B}^{p}, B_{B}^{*}$ is the intersection of the remaining trisector $C A_{A}^{p}$ (proximal to $C A$ ) with $A B_{B}^{P}$ as distal. Then $C_{C}^{*}$ is the intersection of the left trisectors $A C_{C}^{p}$ (proximal to $A B$ ) and $B A_{A}^{p}$ (distal).

If $\alpha=30^{\circ}$ then $A_{A}^{d}$ is not determined. If $30^{\circ}>\alpha$ then $A_{A}^{d}$ is on the same side of $B C$ with $A_{A}^{p}$ and $\angle B A_{A}^{d} C=60^{\circ}-2 \alpha$. If $30^{\circ}<\alpha$ then $A_{A}^{d}$ and $A_{A}^{p}$ are on different sides of $B C$ while $\angle B A_{A}^{d} \mathrm{C}=2 \alpha-60^{\circ}$.

Consider the case $\beta=\gamma$. Then $\triangle B_{B}^{p} A_{A}^{p} B_{B}^{*}=\triangle C_{C}^{p} A_{A}^{p} C_{C}^{*}$ by Corollary 2a. So $A_{A}^{p} B_{B}^{*}=A_{A}^{p} C_{C}^{*}$ and in turn $\triangle A_{A}^{d} A_{A}^{p} B_{B}^{*}=\triangle A_{A}^{d} A_{A}^{p} C_{C}^{*}$. Thus $\triangle A_{A}^{d} B_{B}^{*} C_{C}^{*}$ is isosceles. So if $\triangle A B C$ is equilateral then $\triangle A_{A}^{d} B_{B}^{*} C_{C}^{*}$ is equilateral.

Next consider that $\triangle A B C$ is not equilateral and let $\beta>\gamma$.

Since the location of $A_{A}^{d}$ depends on the value of $\alpha$, assume that $30^{\circ}>\alpha$. Suppose, towards a contradiction that $\triangle A_{A}^{d} B_{B}^{*} C_{C}^{*}$ is equilateral. Let $W$ be the symmetric point of $C_{C}^{*}$ with respect to $A_{A}^{p} A_{A}^{d}$ and let $V$ be

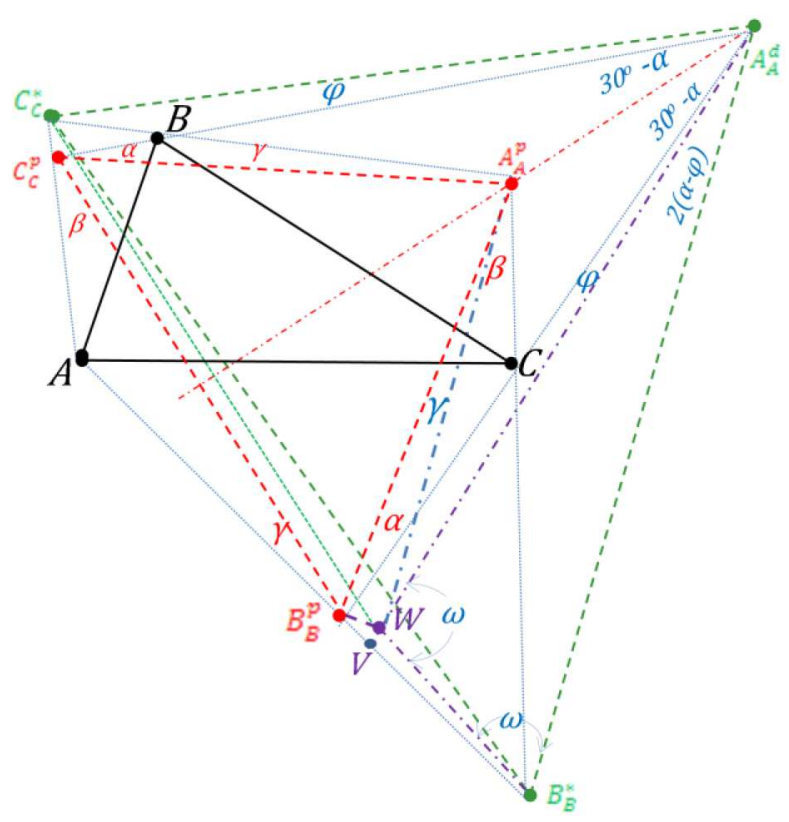

Fig. 22 the intersection of $A_{A}^{d} W$ and $B_{B}^{*} B_{B}^{p}$. In the 
sequel we find the location of $W$.

From Corollary 2b, $A_{A}^{p} A_{A}^{d}$ is height of the equilateral $\triangle A_{A}^{p} B_{B}^{p} C_{C}^{p}$ and so $A_{A}^{p}$ and $B_{B}^{p}$ are symmetric with respect to $A_{A}^{p} A_{A}^{d}$.

Also by Corollary 2a the angles between the trisectors of $\triangle A B C$ and the central Morley equilateral are as depicted in Fig.22. Consequently,

$\angle A_{A}^{\mathrm{d}} C_{C}^{\mathrm{p}} W=\angle A_{A}^{\mathrm{d}} C_{C}^{\mathrm{p}} C_{C}^{*}=180^{\circ}-60^{\circ}-\alpha-\beta=60^{\circ}+\gamma$ and $\angle A_{A}^{\mathrm{d}} \mathrm{B}_{\mathrm{B}}^{\mathrm{p}} \mathrm{B}_{\mathrm{B}}^{*}=180^{\circ}-60^{\circ}-\alpha-\beta=$ $60^{\circ}+\beta$. Therefore $\angle B_{B}^{*} B_{B}^{p} W<\angle A_{A}^{d} B_{B}^{p} B_{B}^{*}$ and the line $B_{B}^{p} W$ is inside $\angle C B_{B}^{p} B_{B}^{*}$.

Furthermore $\angle B_{B}^{p} A_{A}^{p} W=\angle C_{C}^{p} A_{A}^{p} C_{C}^{*}=\gamma$ and since $\beta>\gamma, A_{A}^{p} W$ is inside $\angle B_{B}^{p} A_{A}^{p} B_{B}^{*}$. Thus $W$ is inside $\triangle C B_{B}^{p} B_{B}^{*}$.

Clearly $C_{C}^{*}$ is outside $\triangle A B C_{C}^{p}$. Thus we may set $\angle C_{C}^{p} A_{A}^{d} C_{C}^{*}=\varphi$. Then by symmetry $\angle B_{B}^{p} A_{A}^{d} W=$ $\varphi$. Also set $\angle A_{A}^{d} W B_{B}^{*}=\omega$. Since $\triangle A_{A}^{d} B_{B}^{*} C_{C}^{*}$ is assumed equilateral then $\angle C_{C}^{*} A_{A}^{d} B_{B}^{*}=60^{\circ}$ and so $\angle W A_{A}^{d} B_{B}^{*}=2(\alpha-\varphi)$.

However $A_{A}^{d} B_{B}^{*}=A_{A}^{d} W$ and so $A_{A}^{d} C_{C}^{*}=A_{A}^{d} B_{B}^{*}$ implies $A_{A}^{d} W=A_{A}^{d} B_{B}^{*}$. Thus $\angle W B_{B}^{*} A_{A}^{d}=\omega$ and from $\triangle A_{A}^{d} W B_{B}^{*}, 2 \omega+2(\alpha-\varphi)=180^{\circ} \Leftrightarrow \omega=90^{\circ}+\varphi-\alpha$. Given that $W$ is inside $\triangle C B_{B}^{p} B_{B}^{*}$ infer $\triangle \mathrm{CB}_{\mathrm{B}}^{\mathrm{p}} \mathrm{B}_{\mathrm{B}}^{*}, \omega>\angle \mathrm{A}_{\mathrm{A}}^{\mathrm{d}} \mathrm{VB}_{\mathrm{B}}^{*}$.

But from $\triangle A_{A}^{d} V B_{B}^{*}$ we deduce $\angle A_{A}^{d} V B_{B}^{*}=\angle A_{A}^{d} B_{B}^{p} B_{B}^{*}+\angle B_{B}^{p} A_{A}^{d} V=60^{\circ}+\beta+\varphi$.

Thus $\omega>60^{\circ}+\beta+\varphi \Longrightarrow \omega>60^{\circ}+\beta+\left(\omega+\alpha-90^{\circ}\right) \Longrightarrow \alpha+\beta<30^{\circ}$ which contradicts the assumption $30^{\circ}>\alpha$.

The case $\alpha>30^{\circ}$ is similar and it is omitted.

Conclude that $\triangle A_{A}^{d} B_{B}^{*} C_{C}^{*}$ cannot be equilateral (if $\triangle A B C$ is not equilateral).

\subsection{Morley triangles by trisectors of one interior and two exterior angles}

This section deals with the non equilateral Morley triangles formed by the trisectors of one interior and two exterior angles. Even crude figures of these triangles indicate clearly that they are too asymmetric to be equilaterals. Nevertheless it must be shown rigorously that they are not. We will consider only those formed by the interior trisectors of $\angle \mathrm{C}$ and the exterior trisectors of $\angle \mathrm{A}$ and $\angle \mathrm{B}$, as the other two cases are similar. $\triangle \mathrm{a}_{\mathrm{c}}^{\mathrm{p}} \mathrm{C}_{\mathrm{C}}^{\mathrm{p}} \mathrm{b}_{\mathrm{c}}^{\mathrm{p}}$ denotes the exterior Morley equilateral relative to $\angle \mathrm{C}$.

Notice that the trisectors $A b_{C}^{p}$ and $B a_{C}^{p}$ are parallel iff

$$
\angle \mathrm{a}_{\mathrm{C}}^{\mathrm{p}} \mathrm{BA}+\angle \mathrm{b}_{\mathrm{C}}^{\mathrm{p}} \mathrm{AB}=180^{\circ} \Leftrightarrow 2(\alpha+\gamma)+2(\beta+\gamma)=180^{\circ} \Leftrightarrow \gamma=30^{\circ} .
$$

In this case the distal trisectors with respect to $A B$ do not intersect and hence the distal vertex $C_{C}^{d}$ is not determined. Also if $30^{\circ}>\gamma$ then $C_{C}^{d}$ and $C_{C}^{p}$ are on the same side of $A B$ with $\angle A C_{C}^{d} B=$ $60^{\circ}-2 \gamma$. If $30^{\circ}<\gamma$ then $C_{C}^{d}$ and $C_{C}^{p}$ are on different sides of $A B$ with $\angle A C_{C}^{d} B=2 \gamma-60^{\circ}$. See Fig.23.

Futhermore note that from Corollary 3a the trisectror $\mathrm{Ca}_{C}^{p}$ is inside $\angle C_{C}^{p} a_{C}^{p} B$ and so $\mathrm{Ca}_{C}^{p}$ 
intersects the trisector $B C_{C}^{p}$ between $B$ and $C_{C}^{p}$. Moreover Corollary $3 a$ implies that the extension of $A C_{C}^{p}$ is inside $\angle a_{C}^{p} C_{C}^{p} B$ and so $C a_{C}^{p}$ intersects $A C_{C}^{p}$ inside $\triangle C_{C}^{p} B a_{C}^{p}$. In addition $A C_{C}^{p}$ intesects $B a_{C}^{p}$ between $B$ and $a_{C}^{p}$. Similarly the trisectror $C b_{C}^{p}$ intersects the trisector $A C_{C}^{p}$ between $A$ and $C_{C}^{p}$ and the trisector $B C_{C}^{p}$ inside $\triangle C_{C}^{p} A b_{C}^{p}$. Also $B C_{C}^{p}$ intersects $A b_{C}^{p}$ between $A$ and $b_{C}^{p}$.

\subsubsection{The Morley triangle of distal vertices}

This is denoted by $\triangle a_{C}^{d} C_{C}^{d} b_{C}^{d}$. Vertex $C_{C}^{d}$ is the intersection of the distal to $A B$ trisectors $A b_{C}^{p}$ and $\mathrm{Ba}_{\mathrm{C}}^{\mathrm{p}}$ and is determined iff $\gamma \neq 30^{\circ}$. Vertex $\mathrm{a}_{\mathrm{C}}^{\mathrm{d}}$ is the intersection of the distal to $\mathrm{BC}$ trisectors $B C_{C}^{p}$ and $C b_{C}^{p}$ and hence it is inside $\triangle C_{C}^{p} A b_{C}^{p}$. Vertex $b_{C}^{d}$ is the intersection of the distal to $A C$ trisectors $C a_{C}^{p}$ and $A C_{C}^{p}$ and hence it is inside $\triangle C_{C}^{p} B a_{C}^{p}$. See Fig.23.

Thus, for $\angle \mathrm{C} \neq 90^{\circ} \mathrm{C}_{\mathrm{C}}^{\mathrm{d}}$ is determined while $\mathrm{a}_{\mathrm{C}}^{\mathrm{d}}$ and $\mathrm{b}_{\mathrm{C}}^{\mathrm{d}}$ are inside $\angle A C_{\mathrm{C}}^{\mathrm{d}} \mathrm{B}$. But so $\angle \mathrm{a}_{\mathrm{C}}^{\mathrm{d}} \mathrm{C}_{\mathrm{C}}^{\mathrm{d}} \mathrm{b}_{\mathrm{C}}^{\mathrm{d}}<$ $\angle A C_{C}^{d} B$. However $\angle A C_{C}^{d} B=\left|60^{\circ}-2 \gamma\right|$. Hence $\angle A C_{C}^{d} B<60^{\circ}$. Therefore $\angle a_{C}^{d} C_{C}^{d} b_{C}^{d}<60^{\circ}$.

Conclude $\triangle \mathrm{a}_{\mathrm{C}}^{\mathrm{d}} \mathrm{C}_{\mathrm{C}}^{\mathrm{d}} \mathrm{b}_{\mathrm{C}}^{\mathrm{d}}$ is not equilateral.

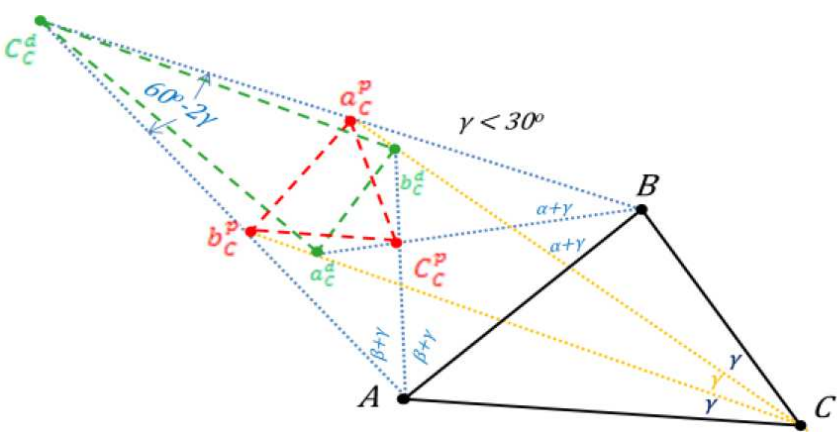

Fig. $23 \mathrm{a}\left(\gamma<30^{\circ}\right)$

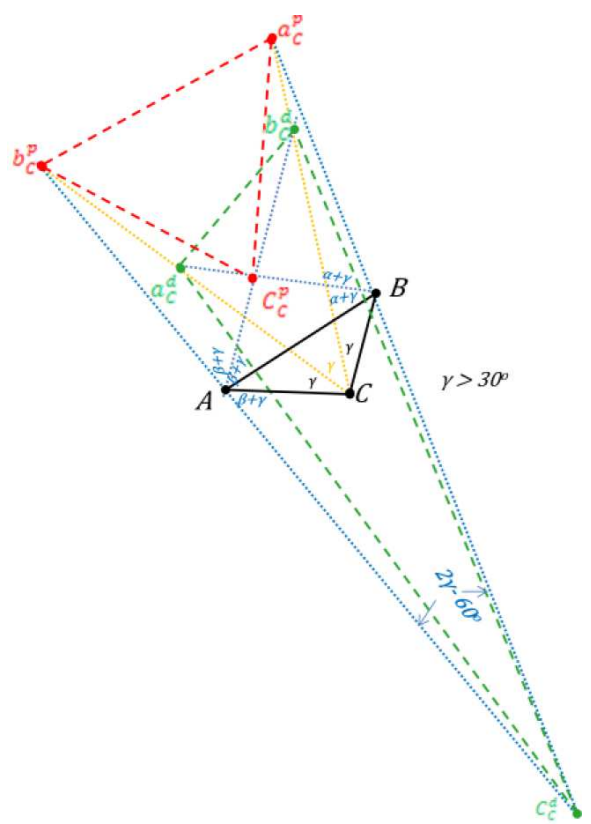

Fig.23b $\left(\gamma>30^{\circ}\right)$

\subsubsection{The Morley triangles with one proximal and two mix vertices}

There are three such triangles denoted by $\triangle a_{C}^{p} b_{C}^{*} C_{C}^{*}, \triangle b_{C}^{p} a_{C}^{*} C_{C}^{*}$ and $\triangle C_{C}^{p} a_{C}^{*} b_{C}^{*}$. 
a. $\triangle a_{C}^{p} b_{C}^{*} C_{C}^{*}$ : Vertex $a_{C}^{p}$ is the intersection of the proximal to $\mathrm{BC}$ trisectors $\mathrm{Ba}_{\mathrm{C}}^{\mathrm{p}}$ and $\mathrm{Ca}_{\mathrm{C}}^{\mathrm{p}}$. Vertex $\mathrm{b}_{\mathrm{C}}^{*}$ must be the intersection of the remaining interior trisector $\mathrm{Cb}_{\mathrm{C}}^{\mathrm{p}}$ (proximal to ) with the exterior trisector $A b_{C}^{p}$, as distal. Hence $C_{C}^{*}$ is the intersection of the left trisectors, $B C_{C}^{p}$ (proximal to $A B$ ) and $A b_{C}^{p}$ (distal). See Fig.24.

So $b_{C}^{*}$ is on $A C_{C}^{p}$ and it is between $A$ and $C_{C}^{p}$. Also $C_{C}^{*}$ is on $A b_{C}^{p}$ and it is between $A$ and $b_{C}^{p}$. No-

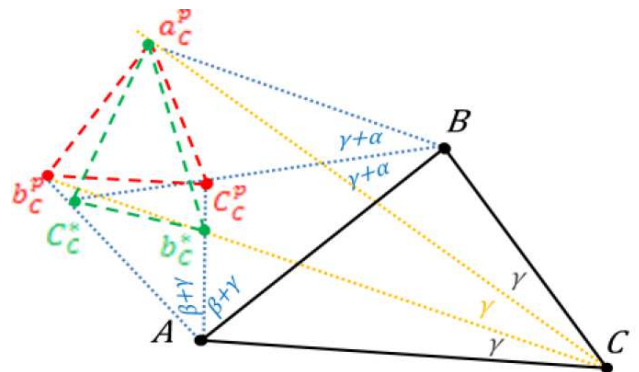

Fig. 24 tice $\angle \mathrm{a}_{\mathrm{C}}^{\mathrm{p}} \mathrm{b}_{\mathrm{C}}^{\mathrm{p}} \mathrm{C}_{\mathrm{C}}^{\mathrm{p}}=\angle \mathrm{a}_{\mathrm{C}}^{\mathrm{p}} \mathrm{C}_{\mathrm{C}}^{\mathrm{p}} \mathrm{b}_{\mathrm{C}}^{\mathrm{p}}=60^{\circ}$ while, by Corollary $3 \mathrm{a}, \angle \mathrm{C}_{\mathrm{C}}^{\mathrm{p}} \mathrm{b}_{\mathrm{C}}^{\mathrm{p}} \mathrm{A}=\alpha+\beta$ and $\angle \mathrm{b}_{\mathrm{C}}^{\mathrm{p}} \mathrm{C}_{\mathrm{C}}^{\mathrm{p}} \mathrm{A}=(\gamma+\alpha)^{+}$. Thus $\angle \mathrm{a}_{\mathrm{C}}^{\mathrm{p}} \mathrm{C}_{\mathrm{C}}^{\mathrm{p}} \mathrm{A}<180^{\circ}$ and $\angle \mathrm{a}_{\mathrm{C}}^{\mathrm{p}} \mathrm{b}_{\mathrm{C}}^{\mathrm{p}} \mathrm{A}<180^{\circ}$. Hence the quadrangle $A b_{C}^{p} a_{C}^{p} C_{C}^{p}$ is convex. Therefore $\angle b_{C}^{*} a_{C}^{p} C_{C}^{*}$ is inside $\angle b_{C}^{p} a_{C}^{p} C_{C}^{p}$ and so $\angle b_{\mathrm{C}}^{*} \mathrm{a}_{\mathrm{C}}^{\mathrm{p}} \mathrm{C}_{\mathrm{C}}^{*}<60^{\circ}$.

Conclude that $\triangle \mathrm{a}_{\mathrm{C}}^{\mathrm{p}} \mathrm{b}_{\mathrm{C}}^{*} \mathrm{C}_{\mathrm{C}}^{*}$ is not equilateral.

b. $\triangle \mathrm{b}_{\mathrm{C}}^{\mathrm{p}} \mathrm{a}_{\mathrm{C}}^{*} \mathrm{C}_{\mathrm{C}}^{*}$ : It is shown as above that it is not equilateral.

c. $\triangle C_{C}^{p} a_{C}^{*} b_{C}^{*}$ : Vertex $C_{C}^{p}$ is the intersection of the proximal to $A B$ exterior trisectors. Thus $\mathrm{a}_{\mathrm{C}}^{*}$ is the intersection of the remaining exterior trisector $\mathrm{Ba}_{\mathrm{C}}^{\mathrm{p}}$ (proximal to $\mathrm{BC}$ ) with the interior trisector $C b_{C}^{p}$, as distal. Then $b_{C}^{*}$ is the intersection of the left trisectors $C a_{C}^{p}$ (distal to $A C$ ) and $A b_{C}^{p}$ (proximal).

So $a_{C}^{*}$ and $C_{C}^{p}$ are on the same side of $B C$ iff

$$
\angle \mathrm{BCb}_{\mathrm{C}}^{\mathrm{p}}+\angle \mathrm{CBa}_{\mathrm{C}}^{\mathrm{p}}<180^{\circ} \Leftrightarrow 2 \gamma+2(\alpha+\gamma)+3 \beta<180^{\circ} \Leftrightarrow \gamma<\alpha .
$$

If $\gamma=\alpha$ then $\mathrm{a}_{\mathrm{C}}^{*}$ is not determined as $\mathrm{Ba}_{\mathrm{C}}^{\mathrm{p}} / / \mathrm{Cb}_{\mathrm{C}}^{\mathrm{p}}$.

Also $b_{C}^{*}$ and $C_{C}^{p}$ are on different sides of $A C$ iff

$$
\angle A C a_{\mathrm{C}}^{\mathrm{p}}+\angle \mathrm{CAb}_{\mathrm{C}}^{\mathrm{p}}<180^{\circ} \Leftrightarrow 2 \gamma+2(\beta+\gamma)+3 \alpha<180^{\circ} \Leftrightarrow \gamma<\beta .
$$

If $\gamma=\beta$ then $b_{C}^{*}$ is not determined as $A b_{C}^{p} / / C a_{C}^{p}$.

Since $a_{C}^{*}, b_{C}^{*}$ and $C_{C}^{p}$ are outside $\triangle A B C$ while $a_{C}^{*}$ and $b_{C}^{*}$ are on $A b_{C}^{p}$ and $B a_{C}^{p}$ respectively we deduce

$$
a_{C}^{*} \text { and } C_{C}^{p} \text { are on the same side of } A B \text { iff } \gamma<\alpha
$$

while

$$
\mathrm{b}_{\mathrm{C}}^{*} \text { and } \mathrm{C}_{\mathrm{C}}^{\mathrm{p}} \text { are on the same side of } \mathrm{AB} \text { iff } \gamma<\beta \text {. }
$$

The above conditions correlate the ranges of $\alpha, \beta C_{C}^{p}$ and $\gamma$ with the different locations of $a_{C}^{*}$ and $b_{C}^{*}$ and vise versa.

Recall that $A b_{C}^{p}$ and $B a_{C}^{p}$ intersect at $C_{C}^{d}$ iff $\gamma \neq 30^{\circ}$, with $\angle A C_{C}^{d} B=\left|60^{\circ}-2 \gamma\right|$, while $C_{C}^{p}$ and $C_{C}^{d}$ are on the same side of $A B$ iff $\gamma<30^{\circ}$.

Next all the different locations of $a_{C}^{*}$ and $b_{C}^{*}$ are considered. 
Case 1: $a_{C}^{*}$ and $b_{C}^{*}$ are on the other side of $A B$ from $C_{C}^{p}$.

This happens iff $\gamma \leq 30^{\circ}$ (and so $\alpha<\gamma$ and $\beta<\gamma$ ) or $\gamma<30^{\circ}$ with $\alpha<\gamma$ and $\beta<\gamma$. Fig.25a,b.

If $\gamma=30^{\circ}$ then $A b_{C}^{p} / / \mathrm{Ba}_{C}^{p}$, while for $\gamma \neq 30^{\circ} A b_{C}^{p}$ and $\mathrm{Ba}_{C}^{p}$ meet at $C_{C}^{d}$.

But so, $a_{C}^{*}$ and $b_{C}^{*}$ are on the extensions (to the other side of $A B$ from $C_{C}^{p}$ ) of $C b_{C}^{p}, B a_{C}^{p}$ and $\mathrm{Ca}_{\mathrm{C}}^{\mathrm{p}}, \mathrm{b}_{\mathrm{C}}^{\mathrm{p}} \mathrm{A}$ respectively.

Since $C_{C}^{p}$ is inside $\triangle a_{C}^{p} C_{C}^{p} b_{C}^{p}$, then $\angle a_{C}^{*} C_{C}^{p} b_{C}^{*}$ encompasses $\angle a_{C}^{*} C b_{C}^{*}$. Therefore $\angle \mathrm{a}_{\mathrm{C}}^{*} \mathrm{C}_{\mathrm{C}}^{\mathrm{p}} \mathrm{b}_{\mathrm{C}}^{*}<\angle \mathrm{a}_{\mathrm{C}}^{*} \mathrm{Cb}_{\mathrm{C}}^{*}$. However $\angle \mathrm{a}_{\mathrm{C}}^{*} \mathrm{Cb}_{\mathrm{C}}^{*}=\gamma$. Deduce $\angle \mathrm{a}_{\mathrm{C}}^{*} \mathrm{C}_{\mathrm{C}}^{\mathrm{p}} \mathrm{b}_{\mathrm{C}}^{*}<60^{\circ}$.

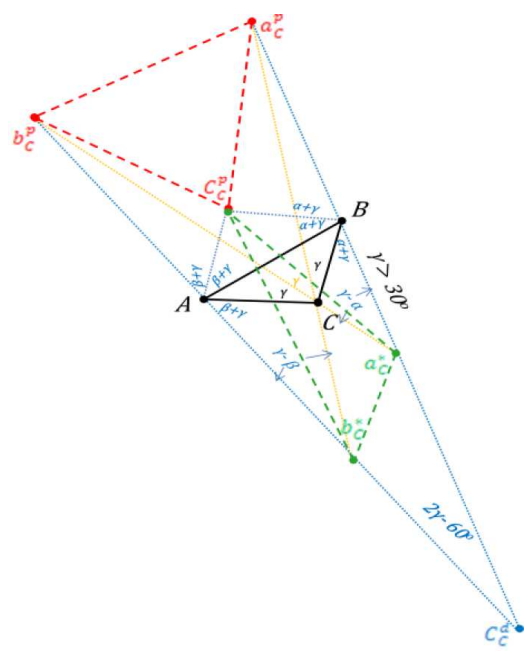

Fig.25a $\left(\gamma>30^{\circ}\right)$

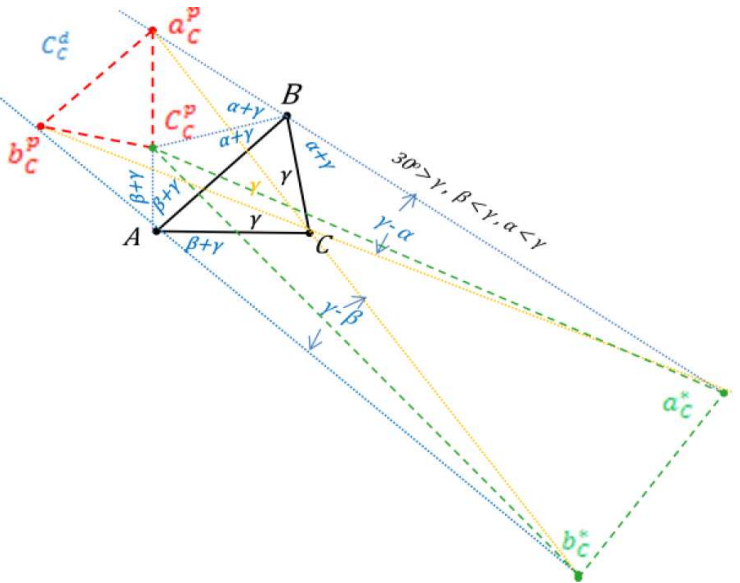

Fig.25b $\left(\gamma<30^{\circ}, \beta<\gamma, \alpha<\gamma\right)$

$\underline{\text { Case } 2}: \mathrm{a}_{\mathrm{C}}^{*}$ and $\mathrm{b}_{\mathrm{C}}^{*}$ are on the same side of $\mathrm{AB}$ with $\mathrm{C}_{\mathrm{C}}^{\mathrm{p}}$.

This happens iff $\gamma<30^{\circ}, \beta>\gamma$ and $\alpha>\gamma$. Fig.25c.

Then $C_{C}^{d}$ and $C_{C}^{p}$ are on the same side of $A B$. Note that $C a_{C}^{p}$ intersects sides $A B$ and $B C_{C}^{d}$ of $\triangle A C_{C}^{d} B$ internally and so, by Pasch's axiom, it intersects the third side $A C_{C}^{d}$ externally. Thus $b_{C}^{*}$ is on the extension of $A C_{C}^{d}$. Similarly $a_{C}^{*}$ is on the extension of $B C_{C}^{d}$. Since $C_{C}^{p}$ is inside $\triangle A C_{C}^{d} B$ then $\angle \mathrm{a}_{\mathrm{C}}^{*} \mathrm{C}_{\mathrm{C}}^{\mathrm{p}} \mathrm{b}_{\mathrm{C}}^{*}$ encompasses $\angle \mathrm{a}_{\mathrm{C}}^{*} \mathrm{C}_{\mathrm{C}}^{\mathrm{d}} \mathrm{b}_{\mathrm{C}}^{*}$. Therefore $\angle \mathrm{a}_{\mathrm{C}}^{*} \mathrm{C}_{\mathrm{C}}^{\mathrm{p}} \mathrm{b}_{\mathrm{C}}^{*}<\angle \mathrm{a}_{\mathrm{C}}^{*} \mathrm{C}_{\mathrm{C}}^{\mathrm{d}} \mathrm{b}_{\mathrm{C}}^{*}$. But $\angle \mathrm{a}_{\mathrm{C}}^{*} \mathrm{C}_{\mathrm{C}}^{\mathrm{d}} \mathrm{b}_{\mathrm{C}}^{*}=$ $\angle A C_{C}^{\mathrm{d}} \mathrm{B}=60^{\circ}-2 \gamma$. Deduce $\angle \mathrm{a}_{\mathrm{C}}^{*} \mathrm{C}_{\mathrm{C}}^{\mathrm{p}} \mathrm{b}_{\mathrm{C}}^{*}<60^{\circ}$.

Case 3: $\mathrm{a}_{\mathrm{C}}^{*}$ and $\mathrm{b}_{\mathrm{C}}^{*}$ are on different sides of AB. Fig.25d.

This happens iff $\gamma<30^{\circ}$ with $\beta>\gamma$ and $\alpha<\gamma$ or with $\beta<\gamma$ and $\alpha>\gamma$.

Next consider the case $\gamma<30^{\circ}$ with $\beta>\gamma$ and $\alpha<\gamma$.

Then $C_{C}^{p}$ is on the same side with $C_{C}^{d}$. Hence $C_{C}^{p}$ is inside $\triangle A C_{C}^{d} B$ and also $C_{C}^{p}$ is inside $\triangle a_{C}^{p} C b_{C}^{p}$. By Pasch's axiom on $\triangle A C_{C}^{d} B$, since $C a_{C}^{p}$ intersects sides $A B$ and $B C_{C}^{d}$ at interior points, infer $\mathrm{Ca}_{\mathrm{C}}^{\mathrm{p}}$ intersects the third side $A C_{C}^{\mathrm{d}}$ at an exterior point. Thus $\mathrm{b}_{\mathrm{C}}^{*}$ is on extensions of $A C_{C}^{\mathrm{d}}$ and $B a_{C}^{p}$ on the same side of $A B$ with $C_{C}^{p}$. So $C_{C}^{p}$ is inside $\triangle b_{C}^{p} C_{C}^{*}$ on the other side of $a_{C}^{p} b_{C}^{p}$ from $C_{C}^{d}$ and 


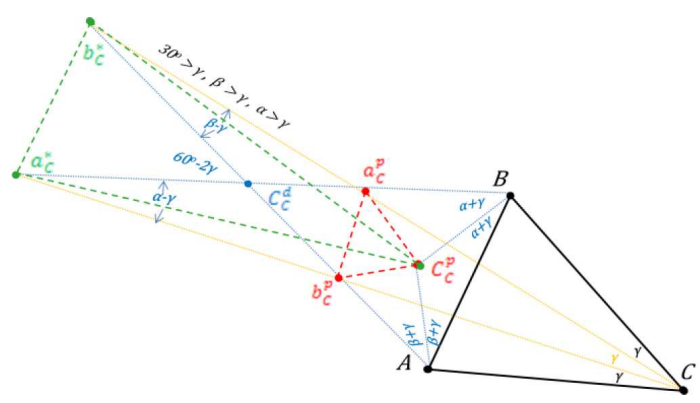

Fig. $25 \mathrm{c}\left(\gamma<30^{\circ}, \beta>\gamma, \alpha>\gamma\right)$

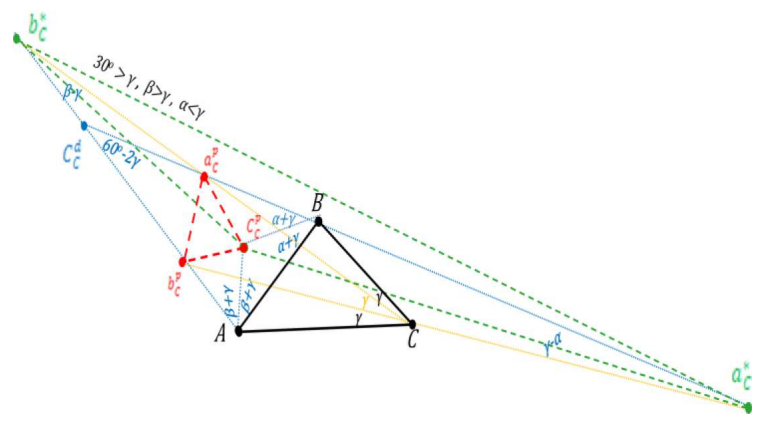

Fig. $25 \mathrm{~d}\left(\gamma<30^{\circ}, \beta<\gamma, \alpha<\gamma\right)$

$b_{C}^{*}$. Consequently $a_{C}^{p} C_{C}^{d}$ intersects $b_{C}^{*} C_{C}^{p}$ between $b_{C}^{*}$ and $C_{C}^{p}$. Hence $a_{C}^{*} a_{C}^{p}$ is inside $\triangle a_{C}^{*} C_{C}^{p} b_{C}^{*}$. Consequently $a_{C}^{p}$ and $B$ are inside $\angle a_{C}^{*} C_{C}^{p} b_{C}^{*}$. Therefore $\angle a_{C}^{*} C_{C}^{p} b_{C}^{*}$ encompasses $\angle a_{C}^{p} C_{C}^{p} B$ and so $\angle a_{C}^{*} C_{C}^{p} b_{C}^{*}>\angle a_{C}^{p} C_{C}^{p} B$.

However Corollary 3a asserts $\angle \mathrm{a}_{\mathrm{C}}^{\mathrm{p}} \mathrm{C}_{\mathrm{C}}^{\mathrm{p}} \mathrm{B}=(\beta+\gamma)^{+}$. Deduce $\angle \mathrm{b}_{\mathrm{C}}^{*} \mathrm{C}_{\mathrm{C}}^{\mathrm{p}} \mathrm{a}_{\mathrm{C}}^{*}>60^{\circ}$.

The case $\gamma<30^{\circ}$ with $\beta>\gamma$ and $\alpha<\gamma$ is similar and it is omitted.

Conclude that $\triangle \mathrm{C}_{\mathrm{C}}^{\mathrm{p}} \mathrm{a}_{\mathrm{C}}^{*} \mathrm{~b}_{\mathrm{C}}^{*}$ is not equilateral.

\subsubsection{The Morley triangles with one distal and two mix vertices}

These Morley triangles are denoted by $\triangle \mathrm{C}_{\mathrm{C}}^{\mathrm{d}} \mathrm{a}_{\mathrm{C}}^{*} \mathrm{~b}_{\mathrm{C}}^{*}, \triangle \mathrm{b}_{\mathrm{C}}^{\mathrm{d}} \mathrm{C}_{\mathrm{C}}^{*} \mathrm{a}_{\mathrm{C}}^{*}$ and $\triangle \mathrm{a}_{\mathrm{C}}^{\mathrm{d}} \mathrm{c}_{\mathrm{C}}^{*} \mathrm{~B}_{\mathrm{C}}^{*}$.

a. $\triangle C_{C}^{d} a_{C}^{*} b_{C}^{*}$ : Vertex $C_{C}^{d}$ is the intersection of the distal to $A B$ trisectors $A b_{C}^{p}$ and $B a_{C}^{p}$. Thus vertex $a_{C}^{*}$ is determined by the intersection of the remaining trisector $\mathrm{C}_{\mathrm{C}}^{\mathrm{P}}$, distal to $\mathrm{BC}$, with $\mathrm{Ca}_{\mathrm{C}}^{\mathrm{p}}$, as proximal. Vertex $b_{\mathrm{C}}^{*}$, is determined by the left trisectors $A C_{C}^{P}$ and $C b_{C}^{p}$ which are distal and proximal to $C A$, respectively.

Vertex $C_{C}^{d}$ is determined iff $\gamma \neq 30^{\circ}$ with $C_{C}^{P}$ and $C_{C}^{d}$ to be on the same side of $A B$ iff $\gamma>30^{\circ}$.

Moreover $a_{C}^{*}$ is always located be-

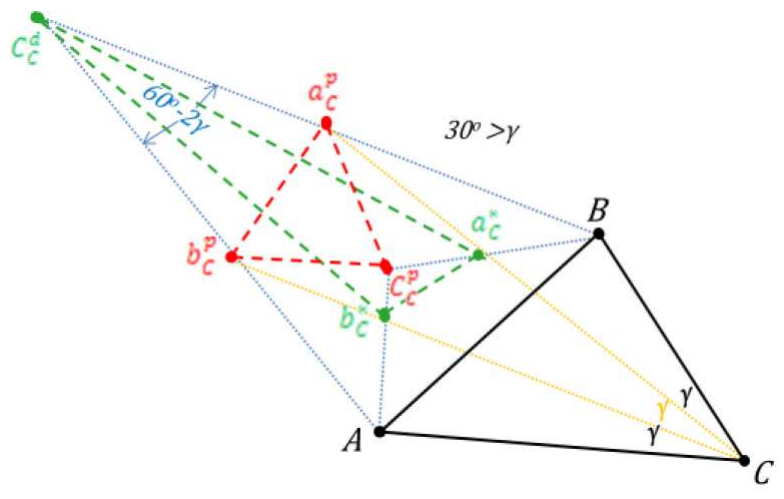

Fig.26 tween $B$ and $C_{C}^{P}$ while $b_{C}^{*}$ is always located between $A$ and $C_{C}^{P}$. Fig.39 depicts the case for $C_{C}^{P}$ and $C_{C}^{d}$ to be on the same side of $A B$. Regardless the location of $\mathrm{C}_{\mathrm{C}}^{\mathrm{d}}$, vertices $\mathrm{a}_{\mathrm{C}}^{*}$ and $\mathrm{b}_{\mathrm{C}}^{*}$ are inside $\angle A C_{C}^{\mathrm{d}} \mathrm{B}$. Thus $\angle \mathrm{a}_{\mathrm{C}}^{*} \mathrm{C}_{\mathrm{C}}^{\mathrm{d}} \mathrm{b}_{\mathrm{C}}^{*}<\angle A C_{C}^{\mathrm{d}} \mathrm{B}$. Since $\angle A C_{C}^{d} B=\left|60^{\circ}-2 \gamma\right|<60^{\circ}$ then $\angle a_{C}^{*} C_{C}^{d} b_{C}^{*}<60^{\circ}$. 
Conclude that $\triangle \mathrm{C}_{\mathrm{C}}^{\mathrm{d}} \mathrm{a}_{\mathrm{C}}^{*} \mathrm{~b}_{\mathrm{C}}^{*}$ is not equilateral.

b. $\triangle b_{C}^{d} C_{C}^{*} a_{C}^{*}$ : Vertex $b_{C}^{d}$ is the intersection of the distal to $C A$ trisectors $C a_{C}^{p}$ and $A C_{C}^{p}$. Hence vertex $C_{C}^{*}$ is determined by the intersection of the remaining trisector $b_{C}^{p}$, distal to $A B$, with $\mathrm{BC}_{\mathrm{C}}^{\mathrm{p}}$, as proximal.

Vertex $a_{C}^{*}$ is determined by the intersection of the left trisectors $C b_{C}^{p}$ and $B a_{C}^{p}$ which are distal and proximal to $\mathrm{BC}$, respectively.

Trisectors $\mathrm{Ca}_{C}^{\mathrm{p}}$ and $A C_{C}^{p}$ always intersect each other and so $b_{C}^{d}$ is located on the same side of $A B$ with $C_{C}^{p}$. Also $A b_{C}^{p}$ and $B C_{C}^{p}$ always intersect each other and so $C_{C}^{*}$ is located on the same side of $A B$ with $C_{C}^{p}$. However $a_{C}^{*}$ is not always determined as $\mathrm{Cb}_{C}^{p} / / \mathrm{Ba}_{\mathrm{C}}^{\mathrm{p}}$ iff $\angle \mathrm{b}_{\mathrm{C}}^{\mathrm{p}} \mathrm{CB}+\angle \mathrm{b}_{\mathrm{C}}^{\mathrm{p}} \mathrm{Ca}_{\mathrm{C}}^{\mathrm{p}}=180^{\circ}$ $\Leftrightarrow \gamma=\alpha$. In fact $a_{C}^{*}$ is on the same side with $b_{C}^{d}$ and $C_{C}^{*}$ iff $\alpha=\gamma$. It should also be noted that $30^{\circ}<\gamma$ implies $\alpha<\gamma$. See Fig.27a.

For establishing that $\triangle b_{C}^{d} a_{C}^{*} C_{C}^{*}$ is not equilateral we will show that $\angle b_{C}^{d} a_{C}^{*} C_{C}^{*}<60^{\circ}$. Recall that $b_{C}^{d}$ is inside $\triangle a_{C}^{p} B C_{C}^{p}$.

Thus $\angle B a_{C}^{*} C_{C}^{*}$ encompasses $\angle b_{C}^{d} a_{C}^{*} C_{C}^{*}$. Hence for proving $\angle b_{C}^{d} a_{C}^{*} C_{C}^{*}<60^{\circ}$ it suffices to show $\angle \mathrm{Ba}_{\mathrm{C}}^{*} \mathrm{C}_{\mathrm{C}}^{*}<60^{\circ}$.

Notice that $\angle \mathrm{Ba}_{\mathrm{C}}^{*} \mathrm{C}_{\mathrm{C}}^{*}=\angle \mathrm{Ba}_{\mathrm{C}}^{*} \mathrm{a}_{\mathrm{C}}^{\mathrm{d}}+\angle \mathrm{a}_{\mathrm{C}}^{\mathrm{d}} \mathrm{a}_{\mathrm{C}}^{*} \mathrm{C}_{\mathrm{C}}^{*}$ and $\angle \mathrm{Ba}_{\mathrm{C}}^{*} \mathrm{a}_{\mathrm{C}}^{\mathrm{d}}=\angle \mathrm{Ba}_{\mathrm{C}}^{*} \mathrm{C}$. From $\triangle \mathrm{Ba}_{\mathrm{C}}^{*} \mathrm{C}$ it is calculated $\angle \mathrm{Ba}_{\mathrm{C}}^{*} \mathrm{C}=|\gamma-\alpha|$ regardless the location of $\mathrm{a}_{\mathrm{C}}^{*}$. Hence $\angle \mathrm{Ba}_{\mathrm{C}}^{*} \mathrm{C}_{\mathrm{C}}^{*}=|\gamma-\alpha|+\angle \mathrm{a}_{\mathrm{C}}^{\mathrm{d}} \mathrm{a}_{\mathrm{C}}^{*} \mathrm{C}_{\mathrm{C}}^{*}$.

Moreover:

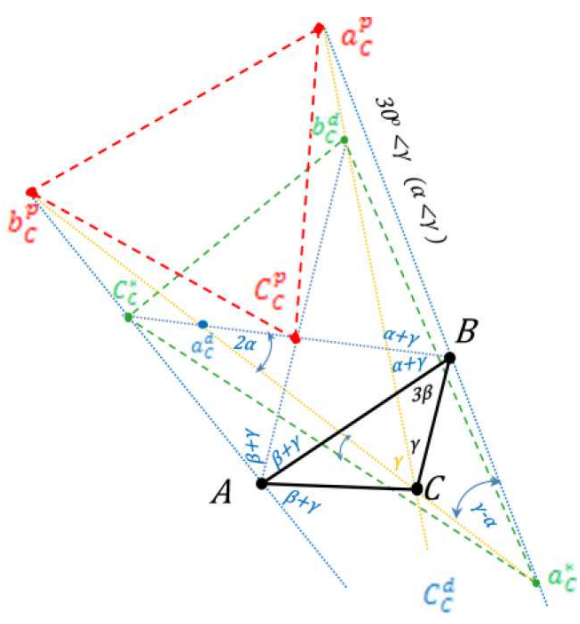

Fig. $27 \mathrm{a}\left(30^{\circ}<\gamma, \alpha<\gamma\right)$

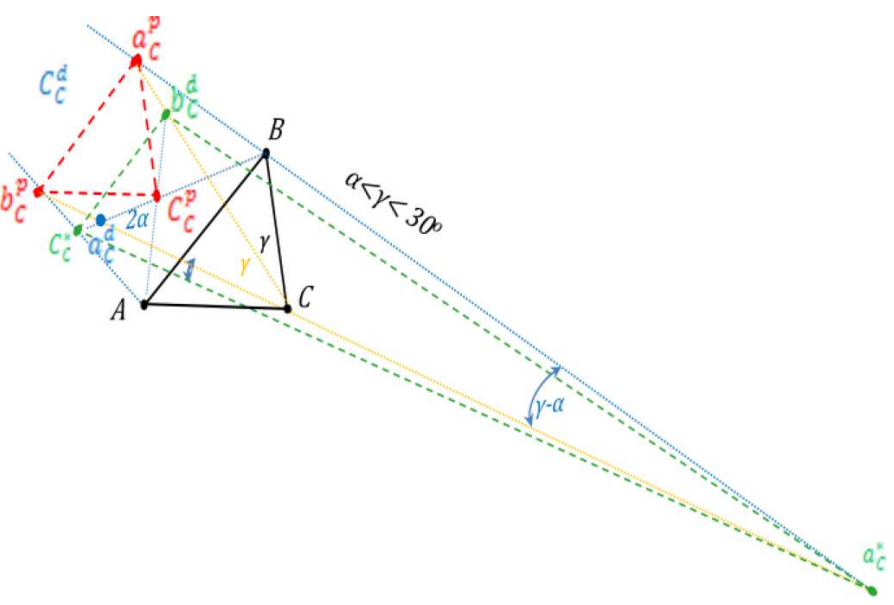

Fig. 27b $\left(\alpha<\gamma<30^{\circ}\right)$

$\triangleright$ If $\alpha<\gamma$ then $a_{C}^{*}$ is on the other side of $A B$ from $a_{C}^{d}$ and $C_{C}^{*}$. See Fig.27a,b. Thus $\angle B a_{C}^{d} C$ is exterior angle in $\triangle a_{C}^{d} a_{C}^{*} C_{C}^{*}$. Hence $\angle a_{C}^{d} a_{C}^{*} C_{C}^{*}<\angle B a_{C}^{d} C$. In $\triangle B a_{C}^{d} C$ it is calculated $\angle C_{C}^{p} a_{C}^{d} C=2 \alpha$ and so $\angle a_{C}^{d} a_{C}^{*} C_{C}^{*}<2 \alpha$. Infer $\angle B a_{C}^{*} C_{C}^{*}<(\gamma-\alpha)+2 \alpha=\gamma+\alpha<60^{\circ}$.

$\triangleright$ If $\gamma<\alpha$ then $a_{C}^{*}$ is on the same side of $A B$, with $a_{C}^{d}$ and $C_{C}^{*}$. See Fig.27c. 


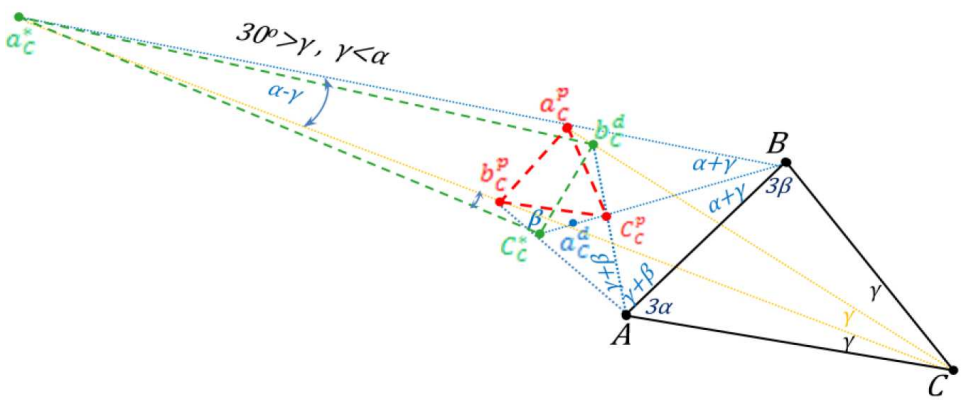

Fig.27c $\left(\gamma<30^{\circ}, \gamma<\alpha\right)$

Thus $\angle \mathrm{a}_{\mathrm{C}}^{\mathrm{d}} \mathrm{b}_{\mathrm{C}}^{\mathrm{p}} \mathrm{C}_{\mathrm{C}}^{*}$ is exterior angle in $\triangle \mathrm{b}_{\mathrm{C}}^{\mathrm{p}} \mathrm{a}_{\mathrm{C}}^{*} \mathrm{C}_{\mathrm{C}}^{*}$. Hence $\angle \mathrm{b}_{\mathrm{C}}^{\mathrm{p}} \mathrm{a}_{\mathrm{C}}^{*} \mathrm{C}_{\mathrm{C}}^{*}<\angle \mathrm{a}_{\mathrm{C}}^{\mathrm{d}} \mathrm{b}_{\mathrm{C}}^{\mathrm{p}} \mathrm{C}_{\mathrm{C}}^{*}$. But $\angle \mathrm{a}_{\mathrm{C}}^{\mathrm{d}} \mathrm{b}_{\mathrm{C}}^{\mathrm{p}} \mathrm{C}_{\mathrm{C}}^{*}=\angle \mathrm{Cb}_{\mathrm{C}}^{\mathrm{p}} \mathrm{A}$. In $\triangle \mathrm{Cb}_{\mathrm{C}}^{\mathrm{p}} \mathrm{A}$ it is calculated $\angle \mathrm{Cb}_{\mathrm{C}}^{\mathrm{p}} \mathrm{A}=\beta$ and so $\angle \mathrm{b}_{\mathrm{C}}^{\mathrm{p}} \mathrm{a}_{\mathrm{C}}^{*} \mathrm{C}_{\mathrm{C}}^{*}<\beta$. Infer $\angle \mathrm{Ba}_{\mathrm{C}}^{*} \mathrm{C}_{\mathrm{C}}^{*}<(\alpha-\gamma)+\beta<60^{\circ}$.

Conclude that $\triangle b_{C}^{d} a_{C}^{*} C_{C}^{*}$ is not equilateral.

c. $\triangle \mathrm{a}_{\mathrm{C}}^{\mathrm{d}} \mathrm{B}_{\mathrm{C}}^{*} \mathrm{c}_{\mathrm{C}}^{*}$ : It is shown as above that it is not equilateral.

\subsection{Morley triangles by trisectors of one exterior and two interior angles}

Eventually the non equilateral Morley triangles formed by trisectors of one exterior and two interior angles are treated. Obviously these Morley triangles have one vertex in the interior and two in the exterior of $\triangle A B C$. As previously we will consider only those formed by the trisectors of the exterior $\angle \mathrm{A}$ combined with the interior trisectors of $\angle \mathrm{B}$ and $\angle \mathrm{C}$ as the other two cases are similar.

\subsubsection{The Morley triangle of proximal vertices}

This is denoted by $\triangle A^{p} b_{A}^{p} c_{A}^{p}$. Vertex $A^{p}$ is the intersection of the proximal to $\mathrm{BC}$ interior trisectors, vertex $b_{A}^{p}$ is the intersection of $C^{p}$ with the exterior trisector of $\angle A$ proximal to $A C$, while vertex $c_{A}^{p}$ is the intersection of $B C^{p}$ with the exterior trisector of $\angle A$ proximal to $A B$.

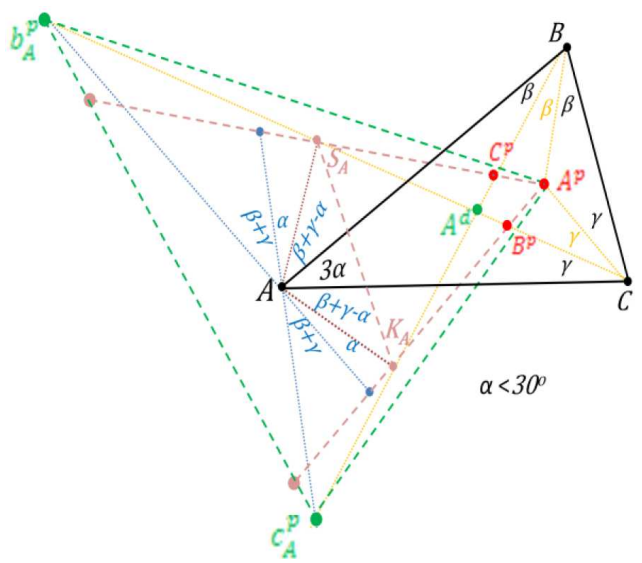

Fig.28 
Consider the companion equilateral $\triangle S_{A} A^{p} K_{A}$ relative to vertex $A$. In Fig. 28 the case $\alpha<30^{\circ}$ is depicted for which Corollary 4 asserts that $\angle B A S_{A}=\angle C A K_{A}=\beta+\gamma-\alpha$ while $S_{A}$ and $K_{A}$ are outside of $\triangle A B C$. Consider the intersections of line $A^{p} C^{p}$ with the sides of $\triangle b_{A}^{p} A^{d} c_{A}^{p}$. Then $A^{p} C^{p}$ intersects side $A^{d} c_{A}^{p}$ at $C^{p}$ and so externally, while it intersects side $A^{d} b_{A}^{p}$ at $S_{A}$ internally since $\angle B A b_{A}^{p}=2(\beta+\gamma)$ and $\angle B A S_{A}=\beta+\gamma-\alpha$. Thus, by Pasch's axiom, $A^{p} C^{p}$ intersects the third side $b_{A}^{p} c_{A}^{p}$ internally. Similarly line $A^{p} B^{p}$ intersects $b_{A}^{p} c_{A}^{p}$ internally. Thus $\angle b_{A}^{p} A^{p} c_{A}^{p}$ encompasses $\angle S_{A} A^{p} K_{A}$ and so $\angle S_{A} A^{p} K_{A}<L_{b}^{p} A^{p} c_{A}^{p}$.

But $\angle S_{A} A^{p} K_{A}=60^{\circ}$ and so $\angle b_{A}^{p} A^{p} c_{A}^{p}>60^{\circ}$. Therefore for $\alpha<30^{\circ}$ the $\triangle A^{p} b_{A}^{p} c_{A}^{p}$ is not equilateral. The cases $\alpha>30^{\circ}$ and $\alpha=30^{\circ}$ are similar.

Conclude that $\triangle A^{p} b_{A}^{p} c_{A}^{p}$ cannot be equilateral.

$\triangleright$ Note that the non equilateral $\triangle A^{p} b_{A}^{p} c_{A}^{p}$ fails the original statement of Morley's theorem.

\subsubsection{The Morley triangle of distal vertices}

This is denoted by $\triangle A^{d} b_{A}^{d} c_{A}^{d}$. Vertex $b_{A}^{d}$ is the intersection of $C A^{p}$, the distal to $C A$ trisector of the interior $\angle C$, and the distal to $C A$ trisector of the exterior $\angle A$. Vertex $c_{A}^{d}$ is the intersection of $B A^{p}$, the remaining trisector of $\angle B$ (distal to $A B$ ) with the distal to $A B$ trisector of the exterior $\angle A$. Also it is easily seen that $b_{A}^{d}$ and $c_{A}^{d}$ are determined iff $\beta \neq \gamma$. Hence, for $\beta \neq \gamma, A^{d}$ is inside $\angle A c_{A}^{d} B$ and $\angle C b_{A}^{d} A$. From $\triangle \mathrm{Cb}_{A}^{\mathrm{d}} A$ it is calculated that

$\angle \mathrm{Cb}_{A}^{\mathrm{d}} A=180^{\circ}-3 \alpha-(\beta+\gamma)-2 \gamma=2 \beta$ and similarly from $\triangle A c_{A}^{d} B, \angle A c_{A}^{d} B=2 \gamma$. Since $\alpha+\beta+\gamma=60^{\circ}$, at least one of $\beta$ and $\gamma$ is less than $30^{\circ}$. Thus either $\angle b_{A}^{d} c_{A}^{d} A^{d}$ or $\angle c_{A}^{d} b_{A}^{d} A^{d}$ is less than $60^{\circ}$.

Conclude that $\triangle A^{d} b_{A}^{d} c_{A}^{d}$ is not equilateral.

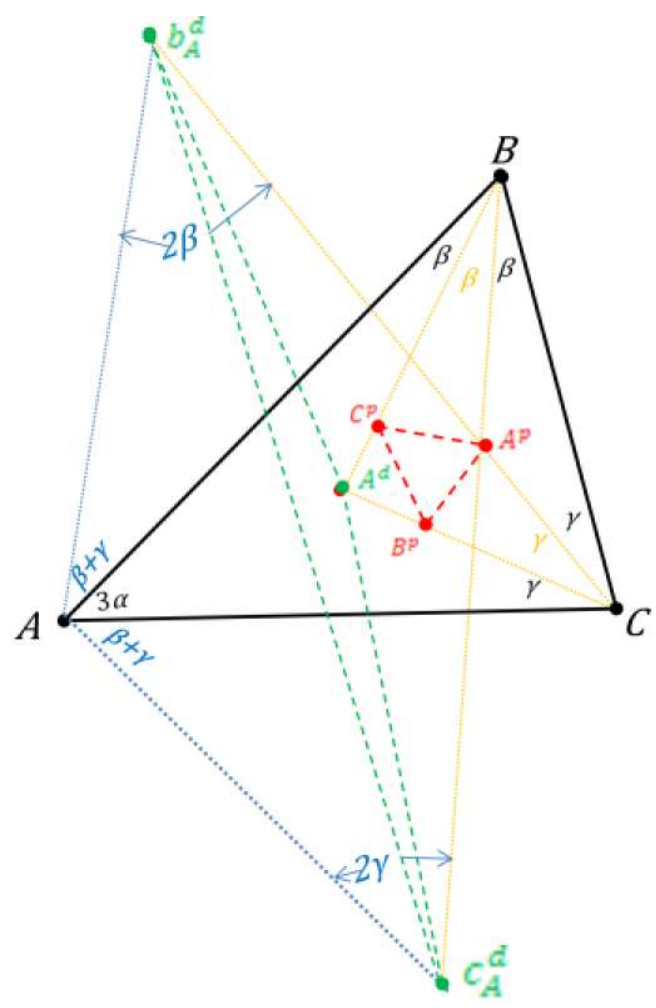

Fig.29 


\subsubsection{The Morley triangles with a proximal and two mix vertices}

These triangles are denoted by $\triangle A^{p} b_{A}^{*} c_{A}^{*}, \triangle b_{A}^{p} A^{*} c_{A}^{*}$ and $\triangle c_{A}^{p} A^{*} b_{A}^{*}$.

a. $\triangle A^{p} b_{A}^{*} c_{A}^{*}$ : Vertex $A^{p}$ is the intersection of the proximal to $B C$ interior trisectors. Hence $b_{A}^{*}$ and $c_{A}^{*}$ are the intersections of the two remaining interior trisectors, $\mathrm{CB}^{p}$ and $\mathrm{BC}^{\mathrm{p}}$, with the trisectors of the exterior $\angle A$. Since each of these interior trisectors is proximal to the side it belongs, it must be paired with the distal to the corresponding side exterior trisector.

Consider the companion equilateral $\triangle S_{A} A^{p} K_{A}$ relative to vertex $A^{p}$. In Fig.30 the case $\alpha>30^{\circ}$ is depicted for which Corollary 4 asserts that vertices $S_{A}$ and $K_{A}$ are inside $\triangle A B C$.

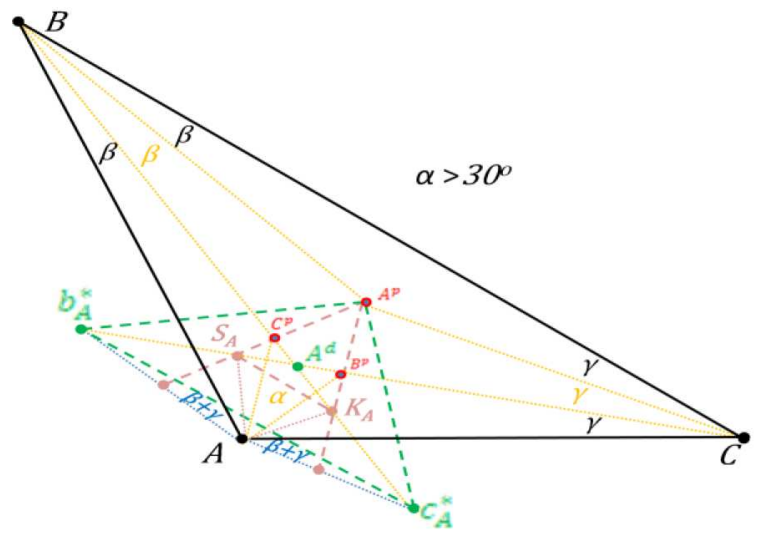

Fig. 30

Consider the intersections of line $A^{p} C^{p}$ with the sides of $\triangle b_{A}^{p} A^{d} c_{A}^{p}$. $A^{p} C^{p}$ intersects side $A^{d} c_{A}^{p}$ at $C^{p}$ and so externally, while $A^{p} C^{p}$ intersects side $A^{d} b_{A}^{p}$ at $S_{A}$ and so internally. Thus, by Pasch's axiom, $A^{p} C^{p}$ intersects the third side $A b_{A}^{*}$ internally. Similarly $A^{p} B^{p}$ intersects $A c_{A}^{*}$ internally. Hence $\angle b_{A}^{p} A^{p} c_{A}^{p}$ encompasses $\angle S_{A} A^{p} K_{A}$ and so $\angle S_{A} A^{p} K_{A}<\angle b_{A}^{p} A^{p} c_{A}^{p}$. But $\angle S_{A} A^{p} K_{A}=60^{\circ}$ and so $\angle b_{A}^{*} A^{p} c_{A}^{*}>60^{\circ}$. Therefore for $\alpha>30^{\circ} \triangle A^{p} b_{A}^{*} c_{A}^{*}$ is not equilateral.

The cases $\alpha<30^{\circ}$ and $\alpha=30^{\circ}$ are similar and they are omitted.

Conclude that $\triangle A^{p} b_{A}^{*} c_{A}^{*}$ is not equilateral.

b. $\triangle b_{A}^{p} A^{*} c_{A}^{*}$ : Vertex $b_{A}^{p}$ is the intersection of the proximal to $A C$ trisectors, which are $C B^{p}$ and the corresponding trisector of the exterior $\angle A$. Thus $A^{*}$ is the intersection of the remaining interior trisector of $\angle C, C A^{p}$, which is proximal to $B C$, with $B C^{p}$ as distal to $B C$. Then $c_{A}^{*}$ is the intersection of the left trisectors $B A^{p}$, which is distal to $A B$, with the proximal to $A B$ trisector of the exterior $\angle A$.

Notice that the last two trisectors are parallel iff $2 \beta=\beta+\gamma \Leftrightarrow \beta=\gamma$. Thus $c_{A}^{*}$ exists iff $\beta \neq \gamma$.

Also if $\beta>\gamma$ then $b_{A}^{p}$ and $c_{A}^{*}$ are on the same side of $A C$, while for $\beta<\gamma, b_{A}^{p}$ and $c_{A}^{*}$ are on different sides of $A C$.

Case $\beta>\gamma$ : We will show $\angle b_{A}^{p} c_{A}^{*} A^{*}<60^{\circ}$. 
Notice that $A^{*}$ is inside $\triangle A B A^{p}$ and so $c_{A}^{*} A^{p}$ is a right bound for the right side $c_{A}^{*} A^{*}$ of $\angle b_{A}^{p} c_{A}^{*} A^{*}$.

In following we will find a left bound for the left side $c_{A}^{*} b_{A}^{*}$ of $\angle b_{A}^{p} c_{A}^{*} A^{*}$.

Let $b_{A}^{*}$ be the intersection of $C A^{p}$ with $A b_{A}^{p}$ and note that the points $A, A^{p}, c_{A}^{*}$, and $b_{A}^{*}$ are cyclic, because from $\triangle B A^{p} C$ it follows that $b_{A}^{*} A^{p} c_{A}^{*}=\beta+\gamma$ and so $b_{A}^{*} c_{A}^{*}$ is seen from $A$ and $A^{p}$ with angle $\beta+\gamma$.

The extension of $A^{p} C^{p}$ meets the exterior trisector $A b_{A}^{*}$ between $A$ and $b_{A}^{*}$. Then it crosses the circle, say at $T$. We will show that a left bound for side $c_{A}^{*} b_{A}^{*}$ of $\angle b_{A}^{p} c_{A}^{*} A^{*}$ is the bisector of $\angle b_{A}^{*} c_{A}^{*} A$.

Note that in a triangle the bisector of an angle crosses its opposite side at a point which is between the side's middle point and the side's common vertex with the shortest of the other two sides.

Let $G$ be the intersection of the $\angle b_{A}^{*} c_{A}^{*} A$ bisector with $A b_{A}^{*}$. Also let $M$ be the middle of $A b_{A}^{*}$.

$\triangle \mathrm{Cb}_{A}^{p}$ is angle bisector in $\triangle A C b_{A}^{*}$. It is easily calculated from $\triangle A C b_{A}^{*}$ that $\angle A b_{A}^{*} C=\beta+\gamma$ and so $\angle A b_{A}^{*} C<\angle b_{A}^{*} A C$. Thus $C A<C b_{A}^{*}$. Hence $b_{A}^{p}$ is between $A$ and $M$.

$\triangleright c_{A}^{*} G$ is angle bisector in $\triangle A b_{A}^{*} c_{A}^{*}$. Obviously $\angle b_{A}^{*} A c_{A}^{*}=\beta+\gamma$. Also $\angle c_{A}^{*} b_{A}^{*} A=\angle c_{A}^{*} b_{A}^{*} c+$ $\angle \mathrm{Cb}_{A}^{*} A$ while $\angle \mathrm{c}_{A}^{*} \mathrm{~b}_{A}^{*} \mathrm{~A}=\angle \mathrm{c}_{A}^{*} \mathrm{~b}_{\mathrm{A}}^{*} \mathrm{C}+\angle \mathrm{Cb}_{A}^{*} A$. But $\angle \mathrm{c}_{A}^{*} \mathrm{~b}_{A}^{*} \mathrm{C}=\angle \mathrm{c}_{A}^{*} A A^{p}=\angle \mathrm{c}_{A}^{*} A B+\angle B A A^{p}=$ $(\beta+\gamma)+\angle B A A^{p}$. So $\angle b_{A}^{*} A c_{A}^{*}<\angle c_{A}^{*} b_{A}^{*} A$ and thus $b_{A}^{*} c_{A}^{*}<b_{A}^{*} A$. Hence $G$ is between $b_{A}^{*}$ and $M$. Therefore $b_{A}^{p}$ is on $A b_{A}^{*}$ and it is between $A$ and $G$. So $\angle G c_{A}^{*} A^{p}$ encompasses $\angle b_{A}^{p} c_{A}^{*} A^{*}$ and thus

$$
\angle \mathrm{b}_{\mathrm{A}}^{\mathrm{p}} \mathrm{c}_{A}^{*} A^{*}<\angle \mathrm{Gc}_{A}^{*} A^{\mathrm{p}}
$$

In the sequel we calculate $\angle \mathrm{Gc}_{A}^{*} A^{p}$. Notice that $\angle \mathrm{Gc}_{A}^{*} A^{p}=\angle \mathrm{Gc}_{A}^{*} A+\angle A c_{A}^{*} A^{p}$ whereas $\angle A c_{A}^{*} A^{p}=\beta-\gamma$ and $\angle G c_{A}^{*} A=\frac{1}{2} \angle b_{A}^{*} c_{A}^{*} A=\frac{1}{2} \angle b_{A}^{*} A^{p} A=\frac{1}{2}\left[\angle b_{A}^{*} A^{p} T+\angle T A^{p} A\right]$.

But

$$
\angle b_{A}^{*} A^{p} T=\angle B A^{p} C^{p}-\angle B A^{p} A^{*}=\gamma^{+}-(\beta+\gamma)=\alpha+\gamma
$$

Also

$$
\angle \mathrm{TAb}_{\mathrm{A}}^{*}=\angle \mathrm{TA}^{\mathrm{p}} \mathrm{b}_{\mathrm{A}}^{*}=\alpha+\gamma \text { and } \angle \mathrm{A}^{\mathrm{p}} \mathrm{TA}=\angle \mathrm{Ac}_{\mathrm{A}}^{*} \mathrm{~A}^{\mathrm{p}}=\beta-\gamma .
$$

Then from $\triangle T A A^{p}$ it is calculated

$$
\angle \mathrm{TA}^{\mathrm{p} A}=\alpha+\gamma-\angle \mathrm{C}^{\mathrm{p}} A \mathrm{~A}^{\mathrm{p}}
$$

Thus

$$
\angle \mathrm{Gc}_{A}^{*} \mathrm{~A}=\alpha+\gamma-\frac{1}{2} \angle \mathrm{TA}^{\mathrm{p}} \mathrm{A}
$$

and so $\angle G c_{A}^{*} A^{p}=\alpha+\beta-\frac{1}{2} \angle C^{p} A A^{p}$ where $0<\angle C^{p} A A^{p}<\alpha$. Hence $\angle G c_{A}^{*} A^{p}<60^{\circ}$. Therefore $\angle b_{A}^{p} c_{A}^{*} A^{*}<60^{\circ}$.

Conclude that for $\beta>\gamma \triangle b_{A}^{p} A^{*} c_{A}^{*}$ is not equilateral. 


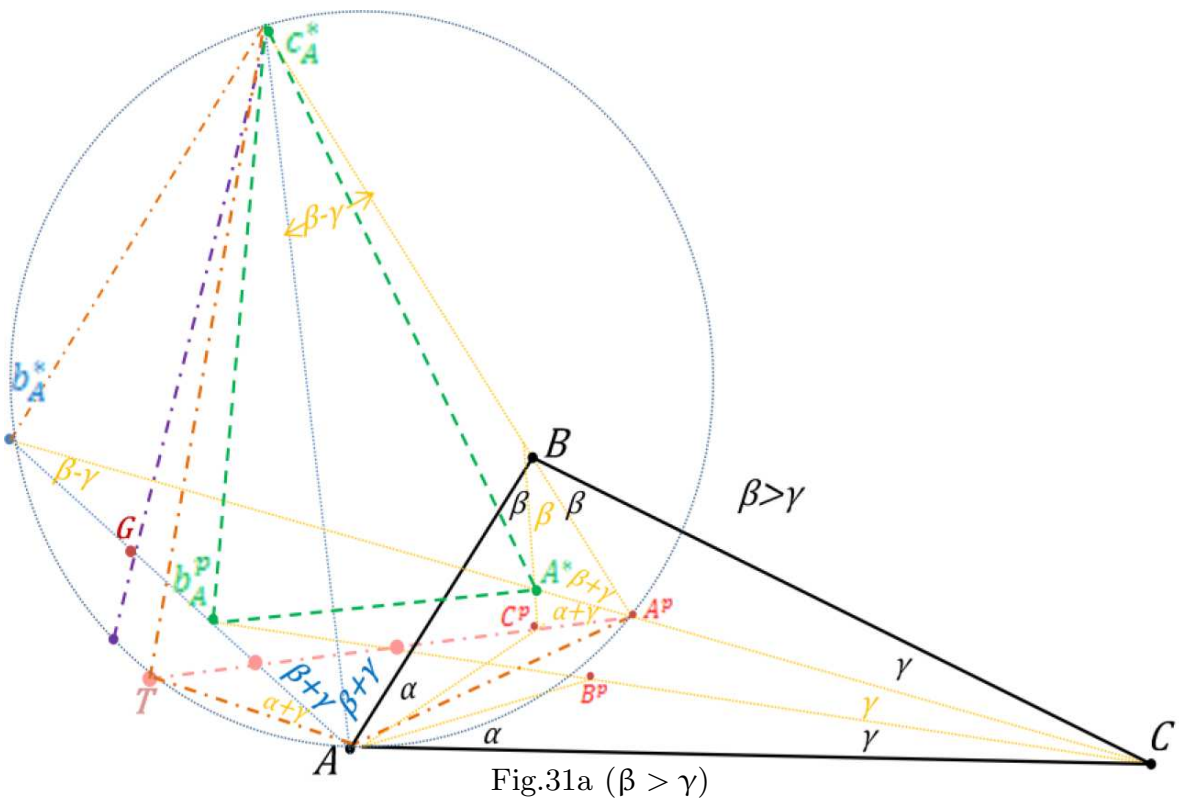

Case $\beta<\gamma$ : We will show that $\angle b_{A}^{P} A^{*} c_{A}^{*}>$ $60^{\circ}$.

Consider the intersections of $A c_{A}^{*}$ with $B C^{p}$ and $C B^{p}$ denoted by $c_{A}^{p}$ and $b_{A}^{*}$ respectively. Notice that $\angle b_{A}^{P} A^{*} c_{A}^{*}$ encompasses $\angle b_{A}^{*} A^{*} c_{A}^{p}$. So it suffices to show that $\angle b_{A}^{*} A^{*} c_{A}^{p}>60^{\circ}$.

Observe that $b_{A}^{*}, A^{*}, C$ and $c_{A}^{p}$ are cyclic, because side $b_{A}^{*} A^{*}$ is seen from $c_{A}^{p}$ and $C$ with angle $\gamma$ as from $\triangle A c_{A}^{p} B$ it is calculated $\angle A c_{A}^{p} B=\gamma$. Thus

$$
\angle b_{A}^{*} A^{*} c_{A}^{p}=\angle b_{A}^{*} C c_{A}^{p} \text {. }
$$

Moreover $\angle b_{A}^{*} C_{A}^{p}=\angle A^{d} C_{A}^{p}$, since $\mathrm{Cb}_{A}^{*}$ passes through $A^{d}$. But

$\angle A{ }^{d} C_{A}^{p}=\angle A{ }^{d} C A+\angle A C c_{A}^{p}=\gamma+\angle A C c_{A}^{p}$ and so

$$
\angle \mathrm{b}_{\mathrm{A}}^{*} \mathrm{Cc}_{\mathrm{A}}^{\mathrm{p}}=\gamma+\angle A C \mathrm{C}_{\mathrm{A}}^{\mathrm{p}} \text {. }
$$

In adition $A, A^{d}, C$ and $c_{A}^{p}$ are also cyclic since $A A^{d}$ is seen from $C$ and $c_{A}^{p}$ with angle $\gamma$.

Consequently $\angle A C c_{A}^{p}=\angle A A^{d} c_{A}^{p}$ and so

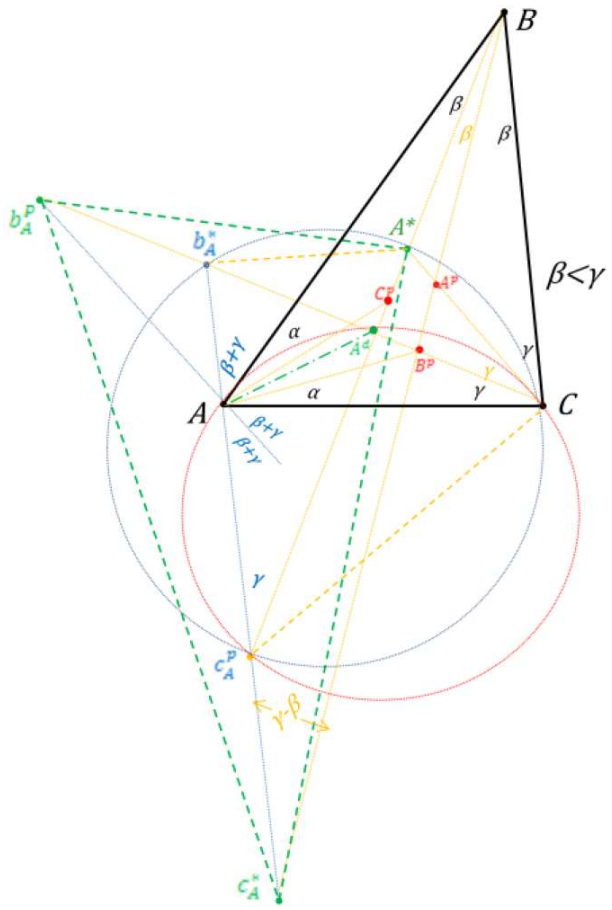

Fig.31b

$$
\angle b_{A}^{*} \mathrm{Cc}_{\mathrm{A}}^{\mathrm{p}}=\gamma+\angle A A^{d} \mathrm{c}_{A}^{p} .
$$

Yet from $\triangle A A^{\mathrm{d}} \mathrm{B}$ infer 


$$
\angle A A^{d} c_{A}^{p}=\angle A B A^{d}+\angle A^{d} A B .
$$

However $\angle A^{\mathrm{d}} A B>\angle C^{p} A B$ and thus

$\angle A A^{d} c_{A}^{p}>\angle A B A^{d}+\angle C^{p} A B=\beta+\alpha$.

Therefore $\angle b_{A}^{*} A^{*} c_{A}^{p}>\gamma+\beta+\alpha=60^{\circ}$.

Conclude that for $\beta<\gamma, \triangle b_{A}^{p} A^{*} c_{A}^{*}$ is not equilateral.

c. $\triangle c_{A}^{p} A^{*} b_{A}^{*}$ : It is showed that it is not equilateral similarly as $\triangle b_{A}^{p} A^{*} c_{A}^{*}$.

\subsubsection{The Morley triangles with one distal and two mix vertices}

These triangles are denoted by $\triangle A^{d} b_{A}^{*} c_{A}^{*}, \triangle c_{A}^{d} A^{*} b_{A}^{*}$ and $\triangle b_{A}^{d} A^{*} c_{A}^{*}$.

a. $\triangle A^{d} b_{A}^{*} c_{A}^{*}$ : Obviously $A^{d}$ is the intersection of $C B^{p}$ and $B C^{p}$. Then $b_{A}^{*}$ is the intersection of the remaining interior trisectors $C A^{p}$ (distal to $A C$ ) with the proximal to $A C$ exterior trisector of $\angle A$. Notice these two lines are parallel iff $\beta+\gamma=2 \gamma \Leftrightarrow \beta=\gamma$. Moreover $c_{A}^{*}$ is the intersection of the left trisectors, the interior $\mathrm{BC}^{\mathrm{P}}$ (proximal) with the distal to $\mathrm{AB}$ trisector of the exterior $\angle A$. Notice these lines are parallel iff $\beta=\gamma$. Therefore $\triangle A^{d} b_{A}^{*} c_{A}^{*}$ is determined iff $\beta \neq \gamma$. From $\triangle A c_{A}^{*} B$ and $\triangle A b_{A}^{*} C$ it follows $\angle A^{p} b_{A}^{*} A=\angle A^{p} b_{A}^{*} A=|\beta-\gamma|$ and so $b_{A}^{*}$ and $c_{A}^{*}$ are on the same side of $A C$.

We will consider only the case $\gamma>\beta$ as the other one is similar.

Notice that $A^{p}$ is inside $\angle B A^{d} C$. Thus

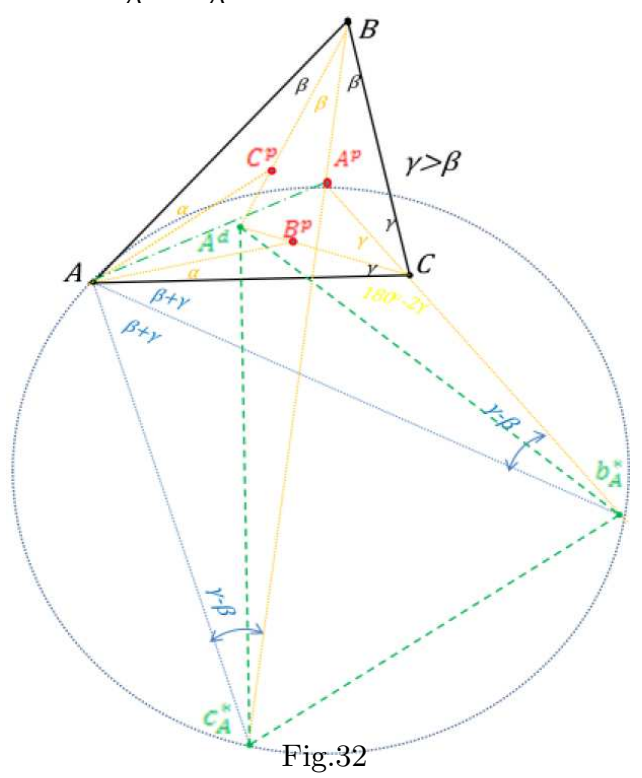
$\angle A^{d} c_{A}^{*} b_{A}^{*}$ encompasses $\angle A^{p} c_{A}^{*} b_{A}^{*}$ and so

$$
\angle A^{d} c_{A}^{*} b_{A}^{*}>\angle A^{p} c_{A}^{*} b_{A}^{*} .
$$

Also notice that $A, A^{p}, b_{A}^{*}$ and $c_{A}^{*}$ are cyclic as $A A^{p}$ is seen from $b_{A}^{*}$ and $c_{A}^{*}$ with angle $\gamma-\beta$. Thus $\angle A^{p} c_{A}^{*} b_{A}^{*}=\angle A^{p} A b_{A}^{*}$. But $\angle A^{p} A b_{A}^{*}=\angle A^{p} A C+\angle C A b_{A}^{*}=\angle A^{p} A C+(\beta+\gamma)$. Moreover $\angle A^{p} A C>\angle B^{p} A C=\alpha$ and so $\angle A^{p} A b_{A}^{*}>\alpha+(\beta+\gamma)=60^{\circ}$. Therefore $\angle A^{d} c_{A}^{*} b_{A}^{*}>60^{\circ}$.

Conclude that $\triangle A^{d} b_{A}^{*} c_{A}^{*}$ is not equilateral.

b. $\triangle b_{A}^{d} A^{*} c_{A}^{*}$ : Obviously $b_{A}^{d}$ is the intersection of $C A^{p}$ with the distal to $A C$ exterior trisector of $\angle A$. Then $A^{*}$ is the intersection of the remaining trisector $C^{p}$ (proximal to $A B$ ) 


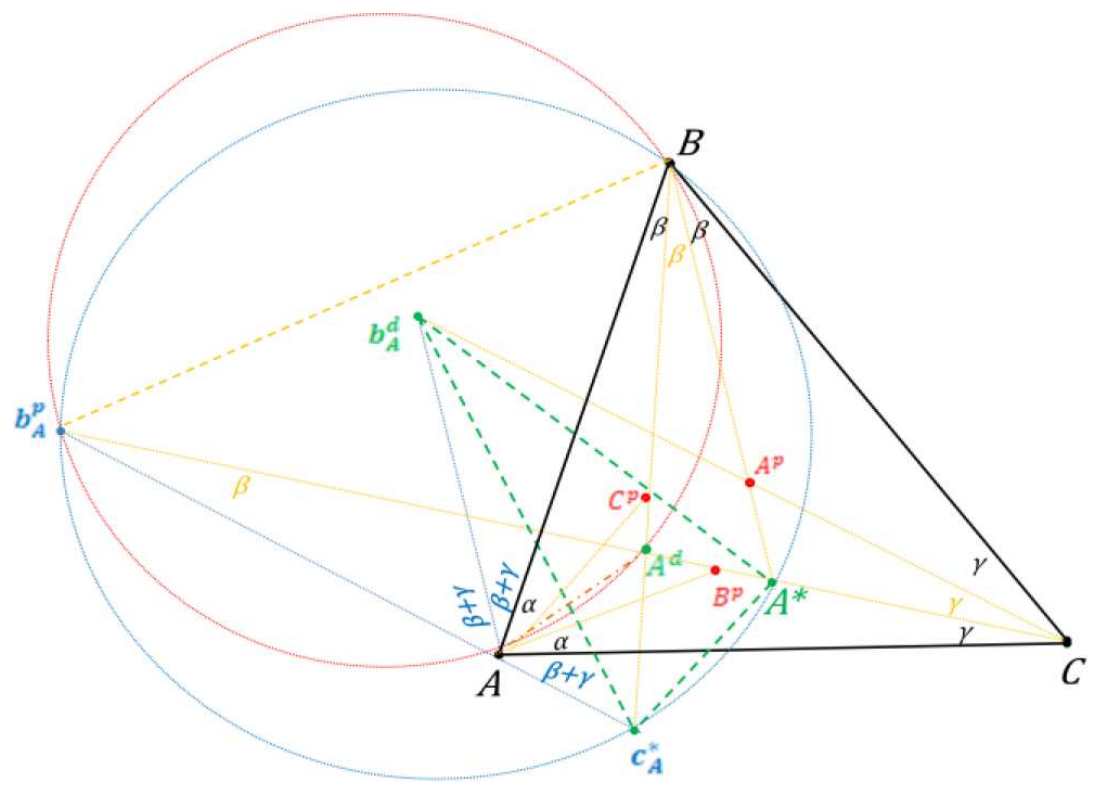

Fig.33

with the $B A^{p}$ (distal). Thus $c_{A}^{*}$ is the intersection of the left trisectors, $B C^{p}$ and the distal to $A B$ exterior trisector of $\angle A$.

Notice that $b_{A}^{d}$ and $c_{A}^{*}$ exist iff $\beta \neq \gamma$. We will show that $\angle b_{A}^{d} A^{*} c_{A}^{*}>60^{\circ}$.

Note that $B^{p}$ is inside $\triangle b_{A}^{d} A^{*} c_{A}^{*}$ and so $\angle b_{A}^{d} A^{*} c_{A}^{*}$ encompasses $\angle b_{A}^{p} A^{*} c_{A}^{*}$. Thus it suffices to prove $\angle b_{A}^{p} A^{*} c_{A}^{*}>60^{\circ}$.

For this we use a symmetric argument to the proof of $\angle b_{A}^{*} A^{*} c_{A}^{p}>60^{\circ}$ (5.4.4.a case $\beta<\gamma$ ).

Let $b_{A}^{p}$ be the intersection of $A c_{A}^{*}$ with $C A^{*}$. Notice that $c_{A}^{*}, A^{*}, B, b_{A}^{p}$ are cyclic as $c_{A}^{*} A^{*}$ is seen from $B$ and $b_{A}^{p}$ with angle $\beta$. Thus $\angle c_{A}^{*} A^{*} b_{A}^{p}=\angle c_{A}^{*} B b_{A}^{p}$. Moreover $\angle c_{A}^{*} A^{*} b_{A}^{p}=$ $\angle A^{d} B b_{A}^{p}$, since $B c_{A}^{*}$ passes through $A^{d}$. But $\angle A^{d} B b_{A}^{p}=\angle A^{d} B A+\angle A B b_{A}^{p}=\beta+\angle A B b_{A}^{p}$ and so $\angle c_{A}^{*} A^{*} b_{A}^{p}=\beta+\angle A B b_{A}^{p}$.

However $A, A^{d}, B, b_{A}^{p}$ are cyclic as $A A^{d}$ is seen from $B$ and $b_{A}^{p}$ with angle $\beta$. Consequently $\angle A B b_{A}^{p}=\angle A A^{d} b_{A}^{p}$ and so $\angle c_{A}^{*} A^{*} b_{A}^{p}=\beta+\angle A A^{d} b_{A}^{p}$.

Yet from $\triangle A A^{d} C$ infer $\angle A A^{d} b_{A}^{p}=\angle A C A^{d}+\angle A^{d} A C$. However $\angle A^{d} A C>\angle B^{p} A B$ and thus $\angle A A^{d} c_{A}^{p}>\angle A C A^{d}+\angle B^{p} A B=\gamma+\alpha$. Therefore $\angle c_{A}^{*} A^{*} b_{A}^{p}>\beta+\gamma+\alpha=60^{\circ}$.

Conclude that $\triangle \mathrm{b}_{A}^{\mathrm{d}} A^{*} \mathrm{c}_{\mathrm{A}}^{*}$ is not equilateral.

c. $\triangle c_{A}^{d} A^{*} b_{A}^{*}$ : This case is similar to the above and it is omitted. 


\section{$6 \quad$ Analogy between Bisectors and Trisectors in a triangle}

The essence of the previous work is portrayed in the following two figures illustrating the analogy between the (well understood) structure of angle bisectors and the (under study) structure of angle trisectors in a triangle.

The structure of angle bisectors

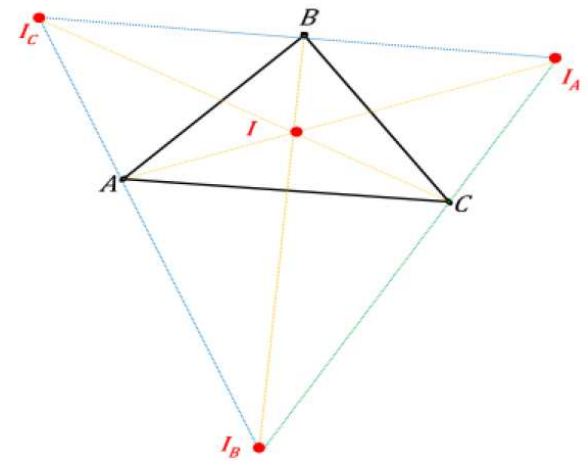

Fig.34a
The structrure of angle trisectors

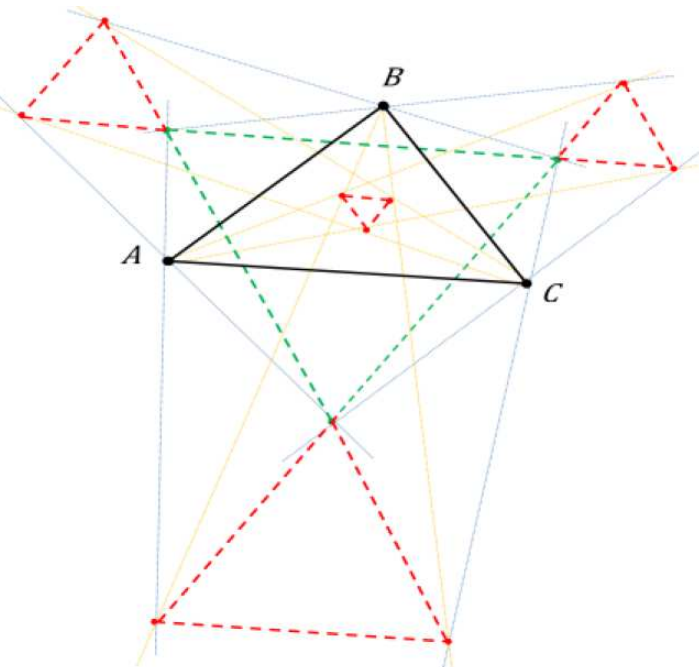

Fig. 34b
The interior angle bisectors pass through a unique point (incenter).

The bisector of an interior angle and the bisectors of the other two exterior angles pass through a unique point (excenter).

The exterior bisectors pass through the vertices of a unique triangle with orthocenter the interior angle bisectors common point (incenter).
The interior angle trisectors proximal to the triangle sides pass through the vertices of a unique equilateral (inner Morley equilateral).

The trisectors of an interior angle and the trisectors of the other two exterior angles proximal to the triangle sides pass through the vertices of a unique equilateral (exterior Morley equilateral).

The exterior trisectors proximal to the triangle sides pass through the vertices of a unique equilateral (central Morley equilateral). ${ }^{+}$

\footnotetext{
+ This fact follows from the previous one
} 
This structural similarity suggests that the triangle trisectors with the proper pairing meet at equilaterals which correspond to the triangle bisectors common points. The perception that trisectors behave like bisectors with equilaterals instead of points invites further exploration. New results could be inspired from the vast variety of the angle bisectors' point-line-circle theorems revealing more exciting analogies between the two structures.

This work owes gratitude to John Conway, Gerry Ladas, George Metakides, Stanley Tennenbaum, Thanasis Fokas, Fotis Fragos and foremost to Frank Morley. Its title is the instantaneous Ladas response to the showing of the last figure, when the project was trying to take off. If it revealed any of the Morley triangles' mystery hopefully it has left their charm untouched.

Received: January 2014. Revised: April 2014.

\section{References}

[1] Alain Connes, A new proof of Morley's theorem, Les relations entre les mathématiques et la physique théorique: Festschrift for the 40th anniversary of the IEÉS. Institut des hautes études scientifiques (1998), 43-6.

[2] John Conway, The power of mathematics, in Power, Cambridge University Press, (2006), 36-50.

[3] Edsger Dijkstra, An open letter to Ross Hornsberger, (1975) E. W. Dijkstra Archive (EWD544).

[4] Richard L. Francis, Modern Mathematical Milestones: Morley's Mystery, Missouri Journal of Mathematical Sciences 14 (1), (2002).

[5] Richard K. Guy, The Lighthouse Theorem A Budget of Paradoxes, Amer. Math. Monthly, 141 (2007) 97-141.

[6] Ross Honsberger, Mathematical Gems I, Dolciani Math. Explosions 1, Math. Assoc. Amer., (1973).

[7] R. C. Lyness, Angles Circles and Morley's Theorem, in Mathematical Reflections, eds Members of the Association of Teachers of Mathematics, Cambridge University Press, (1968).

[8] C.O. Oakley, J.C. Baker, The Morley trisector theorem, The Amer. Math. Monthly,(1978) JSTOR.

[9] R. Penrose, Morley's Trisector Theorem, Eureka 16, (1953), 6-7.

[10] Haim Rose, A simple proof of Morley's theorem, Amer. Math. Monthly, 71, (1964) 771-773. (Is it a proof?) 
[11] W. R. Spickerman, An Extension of Morley's Theorem, Mathematics Magazine-JSTOR, 44, (1971) 191-192.

[12] Glanville F. Taylor and L.W. Marr, The six trisectors of each of the angles of a triangle, Proceedings of the Edinburgh Mathematical Society, 33 ,(1913-14) 119-131.

[13] Cut the Knot, Morley's Miracle, http://www.cut-the-knot.org/triangle/Morley/index.shtml

[14] Facultad CC. Matemáticas, Universidad Complutense de Madrid, Experimenting with Morley's triangle, http://www.mat.ucm.es/catedramdeguzman/drupal/sites/default/files/mguzman/ 08sabormat/geometriatriangulo/DiscoveryExperienceswithDERIVE/AroundMorley.html 